\title{
SELFCONSISTENT SEMICLASSICAL DESCRIPTION OF AVERAGE NUCLEAR PROPERTIES - A LINK BETWEEN MICROSCOPIC AND MACROSCOPIC MODELS
}

\author{
M. BRACK
}

Institut für Theoretische Physik, Universität Regensburg, D-8400 Regensburg, W.-Germany

$$
\text { C. GUET }
$$

Département de Recherche Fondamentale, DPN, CENG, 85 X, F-38041 Grenoble, France

and

\section{H.-B. HÅKANSSON}

Institut für Theoretische Physik, Universität Regensburg, D-8400 Regensburg, W.-Germany

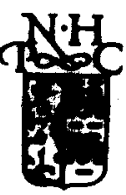

NORTHHOLAMD-AMSTERDAM 


\title{
SELFCONSISTENT SEMICLASSICAL DESCRIPTION OF AVERAGE NUCLEAR PROPERTIES - A LINK BETWEEN MICROSCOPIC AND MACROSCOPIC MODELS ${ }^{\dagger}$
}

\author{
M. BRACK \\ Institut für Theoretische Physik, Universität Regensburg, D-8400 Regensburg, W-Germany \\ C. GUET \\ Département de Recherche Fondamentale, CPN, CENG, 85 X, F-38041 Grenoble, France \\ and \\ H.-B. HÅKANSSON* \\ Institut für Theoretische Physik, Universität Regensburg, D-8400 Regensburg, W.-Germany \\ Received November 1984
}

To Lis, Anna and Frederika

Contents:

1. Introduction

2. Justification of the semiclassical approach based on the microscopical Skyrme-Hartree-Fock formalism

2.1. The Skyrme-HF energy density

2.2. Separation of shell effects

2.3. Strutinsky-averaging as a microscopical link to the ETF model

2.4. Summary

3. The extended Thomas-Fermi model

3.1. The Wigner-Kirkwood expansion

3.2. The ETF functionals $\tau[\rho]$ and $J[\rho]$

3.3. Density variation method and discussion of the ETF Euler equations

4. Semiclassical variational calculations for finite nuclei

4.1. Parametrisation of the nuclear densities
277

280

280

284

285

286

286

286

288

290

295

295
4.2. Bulk properties of spherical nuclei

4.3. Deformation energies and fission barriers of heavy nuclei

4.4. Adjustment of the force SkM*

4.5. Perturbative inclusion of shell effects

5. Liquid drop model type expansion of the ETF binding energy

5.1. Leptodermous expansion of the energy for symmetric nuclei

5.2. Semi-infinite nuclear matter calculations

5.3. Discussion of the droplet model

6. Extension to nuclear systems at finite temperature

6.1. Earlier approaches

6.2. The ETF model at finite temperature

6.3. Discussion of the ETF Euler equation at $T>0$
297

303

308

309

311

311

318

323

328

329

330

337

${ }^{\dagger}$ Work partially supported by Deutsche Forschungsgemeinschaft ( $\left.\mathrm{Az} \mathrm{Br} 733 / 1,2-2\right)$.

* Present address: ASEA STAL AB, S-61220 Finspång, Sweden.

Single orders for this issue

PHYSICS REPORTS (Review Section of Physics Letters) 123, No. 5 (1985) 275-364.

Copies of this issue may be obtained at the price given below. All orders should be sent directly to the Publisher. Orders must be accompanied by check.

Single issue price Dfl. 58.00, postage included.

0 370-1573/85/\$31.50 @ Elsevier Science Publishers B.V. (North-Holland Physics Publishing Division) 
6.4. Test of the TETF functionals against HF results

6.5. Temperature dependence of fission barriers and LDM parameters

7. Summary

Appendices

A. Fourth order gradient corrections to the ETF functionals

B. Expectation values of two-body operators in the ETF approach
342

345

349

350

351
C. Intrinsic curvilinear coordinate system and leptodermous expansion of moments of the density

D. Leptodermous expansion of the Coulomb energy $\quad 357$ Acknowledgements 361

References $\quad 362$

Note added in proof $\quad 364$

\begin{abstract}
:
We present variational semiclassical calculations of average nuclear properties, starting from Skyrme effective nucleon-nucleon interactions. Within the Hartree-Fock (HF) framework, the microscopical justification of the use of the extended Thomas-Fermi (BTF) model and the perturbative treatment of shell effects are briefly reviewed. After a discussion of the ETF functionals. for the kinetic energy and spin-orbit densities and of the Euler variational equation, results for average binding energies, densities and radii as well as fission barriers are presented. The close agreement with results of averaged HF calculations and the validity of the perturbative inclusion of shell effects are demonstrated. The semiclassical binding energies are analysed by means of a "leptodermous" expansion in powers of $A^{-1 / 3}$, and herewith a link between the Skyrme forces and the parameters of the liquid drop model (LDM) and its extensions is given. We discuss in particular the droplet model by Myers and Swiatecki and possible extensions, coming to the conclusion that the variational ETF formalism with its 8-10 Skyrme force parameters is more powerful than the droplet model in its present form. Finally, the ETF formalism is extended to finite temperatures and excellently confirmed by results of HF calculations for heated nuclei. It is then applied to study the temperature dependence of fission barriers and LDM parameters pertinent to astrophysical applications.
\end{abstract}

\title{
1. Introduction
}

Over the last decade, the microscopical description of nuclear ground-state and low-energy excitation properties within the Hartree-Fock (HF) framework has made a considerable progress.

This progress is mainly due to the development of phenomenological effective nucleon-nucleon interactions which may be understood as mathematically simple parametrisations of a density-dependent effective $G$-matrix. (For a review of such effective forces and their applications in HF calculations, see ref. [1].) The most simple of these effective forces, and most widely used, is that of Skyrme [2] in its revived form by Vautherin and Brink [3]. It has mathematically a zero range; however, velocitydependent terms mock up the finite range of the nuclear force. This allows to write the nuclear part of the HF energy as a functional of local one-body densities only, and the HF equations take the form of simple Schrödinger equations with local mean fields (including variable effective nucleon masses and spin-orbit potentials).

Due to their simplicity the Skyrme forces led, with some numerical effort, to the first successful HF calculations of fission barriers of heavy nuclei [4]. However, the actual heights of the barriers turned out to be too large by roughly a factor of two compared with experiment. Similar results were later obtained with other effective forces, too; in fact it remained a puzzle until very recently, why these forces, which otherwise gave very good fits of nuclear ground-state properties both for spherical and deformed nuclei, all led to too high fission barriers. (For a review on the status of fission barrier calculations up to 1979, see ref. [5].) An obvious attempt to answer this question would have been a new fit of the force parameters, taking ground-state properties and fission barriers into account. This was, however, practically impossible due to the excessive computer times required by such $\mathrm{HF}$ calculations.

This dilemma provided a strong motivation to develop semiclassical methods as an efficient tool to obtain selfconsistent average nuclear binding and deformations energies. The so far most successful semiclassical method for this purpose makes use of the local density functionals $\tau[\rho]$ and $J[\rho]$ for the kinetic energy and spin-orbit density, respectively, derived from the so-called extended Thomas-Fermi 
(ETF) model which was revived in the context of nuclear physics some 14 years ago by Bhaduri and Ross [6]. These functionals allow to express the average part of the Skyrme-HF energy as a functional of the local proton and neutron densities and thus eliminating completely the single-particle wave functions.

It is this type of semiclassical variational calculations, their justification and some of their applications, which form the content of this paper. They led to the determination of the first effective nucleon force, labeled $\mathrm{SkM}^{*}$, which gives realistic fission barriers of actinide nuclei and at the same time excellent ground-state properties of stable nuclei. Microscopical calculations with this force have already been published in a recent paper [7]; the semiclassical barrier calculations and the way in which the force $\mathrm{SkM}^{*}$ has been adjusted will be discussed in section 4 of the present paper.

The idea of expressing the total binding energy of a nucleus as a functional of the local density $\rho(r)$-ignoring for the moment the difference between neutrons and protons - and to formulate with it a variational principle

$$
\frac{\delta}{\delta \rho} \int \mathrm{d}^{3} r\{\mathscr{E}[\rho(\boldsymbol{r})]-\lambda \rho(\boldsymbol{r})\}=0
$$

has, in fact, been used early in the history of nuclear physics in the pioneering work which led to the famous semi-empirical Bethe-Weizsäcker mass formula [8,9]. Sophistication of the energy functional $\mathscr{E}[\rho]$ was developed along with the understanding of the nature of the nuclear force $[2,10,11]$ and led to the so-called energy density formalism $[12,13]$. The theoretical justification of the variational approach eq. (1.1) was given only relatively late in this development, in form of the now well-known theorem by Hohenberg and Kohn [14], and came from outside nuclear physics.

The energy density variational calculations performed up to 1973 have been reviewed by Lombard [13]. Typically, the experimental binding energies of spherical nuclei could be reproduced to within $\sim 1-10 \mathrm{MeV}$ and their radii within $\sim 1-4 \%$. (The shell effects, which cannot be reproduced in such a formalism, contribute about $\pm(1-15) \mathrm{MeV}$ to the total binding energy and well below $1 \%$ to the radii.) The density profiles obtained with these calculations were as a rule rather poor. The deficiencies can be traced back mainly to the use of an insufficient kinetic energy density functional-mostly, the TF relation $\tau \sim \rho^{5 / 3}$ was used, sometimes a gradient correction with an adjustable coefficient was added. The corresponding large errors in the kinetic energies were partially made up by the readjustment of the nuclear force parameters, but this cancellation could not help to improve the resulting density profiles.

The recent development of the Skyrme forces on one hand and the refinement reached in the ETF functionals $\tau[\rho]$ and $J[\rho]$-adapted to the use of nonlocal Skyrme type one-body Hamiltonians $[15,16]-$ on the other hand, which took place in about the same time, make it possible now to reassess the energy density formalism in a much more rigorous way. Furthermore, the use of microscopically Strutinsky-averaged HF results [17] allows to test and confirm the semiclassical calculations to a high accuracy, as shall be demonstrated explicitly in this paper. The main strategy of the new approach [18] is:

1) to use a Skyrme force as determined in HF calculations without touching its parameters,

2) to use the density functionals $\tau[\rho]$ and $\boldsymbol{J}[\rho]$ as determined once and for all from the ETF model, without readjusting any of its parameters, and

3) to determine the nucleon densities $\rho_{\mathrm{p}}(r)$ and $\rho_{\mathrm{n}}(\boldsymbol{r})$ variationally and thus to calculate the average nuclear properties of interest without any adjustable variable. 
4) The shell effects, wherever they are of importance, can be added perturbatively $[17,18]$ in terms of the average mean fields.

In this way, the semiclassical results can always be tested against averaged HF results and possible deficiencies of the density functionals can be disentangled from possible deficiencies of the Skyrme force itself.

One might ask what the use of such calculations will be in an era of new computer generations, where even the determination of the fission barrier of a heavy nucleus by the constrained HF method may be a matter of minutes only. Even then, we believe that a time gaining factor of $50-100$ or more will still be of interest in many cases, in particular for searches of new force parameter sets or for systematic calculations of many nuclear species (e.g. calculation of mass tables). Besides this practical motivation, the following are more physical arguments.

1) Transparency:

A density variational calculation allows more physical insight into the way in which different nuclear bulk properties are connected to each other and how they depend on various ingredients of the force, than this is the case for a HF calculation which to a certain extent is like a black box. This becomes particularly evident when establishing the

2) link between the effective force and the liquid drop model (LDM):

The liquid drop model, and its extensions such as the droplet model by Myers and Swiatecki [19], can in fact be formally derived from the variational ETF model by means of a "leptodermous expansion", as will be discussed extensively in section 5 of this paper. Thus, the LDM or droplet model parameters for a given Skyrme force can be determined-some of them analytically - and used in simpler model calculations. Parametrizing in a similar way the average mean fields resulting from a semiclassical calculation, one obtains a

3) selfconsistent input for Strutinsky calculations:

The shell-correction method [20] provides an efficient and rather accurate approximation to the constrained HF method, in particular if the average (LDM) energy and the average (shell model) potentials have been derived selfconsistently from the same effective force [17]. This can precisely be achieved in semiclassical variational calculations.

4) Application to hot nuclear systems:

Excited nuclear systems with temperatures larger than $\sim 3 \mathrm{MeV}$ contain no more shell effects and are thus ideal objects for semiclassical investigations. Such hot nuclear compound systems can be produced in heavy ion and high-energy hadron induced reactions [21]. In astrophysics one is interested in the equation of state of hot nuclear matter. In particular, a possible phase transition between nuclei and bubbles in hot dense matter may play a crucial role in the evolution of supernovae [22]. Although HF calculations can be performed [23] for such systems, too, it seems more appropriate and efficient to use semiclassical techniques, not least because such calculations have to be done over and over as the effective forces - and especially their asymmetry properties - are further improved.

The aim of this paper is not to produce systematic results of nuclear bulk properties, but rather to present the semiclassical variational method itself, to discuss its justification and to demonstrate its validity in some typical applications, testing the results against HF calculations wherever available.

The paper is organized as follows: section 2 deals with the basic justifications of the use of semiclassical methods and of the perturbative treatment of shell effects, as they can be given from the microscopical HF point of view, making use of the so-called Strutinsky energy theorem. In section 3 we present details of the ETF model and discuss in particular the corresponding gradient expansions of the density functionals $\tau[\rho]$ and $\boldsymbol{J}[\rho]$. Section 4 contains the bulk of the variational semiclassical cal- 
culations of nuclear ground-state properties and deformation energies (in particular, fission barriers) and their comparison with HF calculations, and discusses the perturbative inclusion of shell effects.

We use in this paper different sets of Skyrme force parameters existing in the literature, in particular however the force $\mathrm{SkM}^{*}$ whose determination is discussed in section 4.4. In section 5 we deal with the derivation of LDM-like expansions of the semiclassical binding energy. We shall present variational calculations for (symmetric and asymmetric) semi-infinite nuclear matter from which most of the basic droplet model parameters can be determined. We shall study the ways of extracting them from finite nuclei and discuss the basic problem of the asymptotic nature of expansions in powers of $A^{-1 / 3}$. Several shortcomings of the droplet model in its present form are discussed, and some ways of improving it will be proposed. In section 6 we present extensions of the ETF model to finite temperatures. We discuss in particular in section 6.2 the newly derived exact temperature dependent (TETF) functionals which include also a gradient correction $\sigma_{2}[\rho]$ to the entropy density. We shall present the proof that the standard ETF functionals for $T=0$ (used and discussed in sections 3-5) are strictly valid everywhere in space, including the classically forbidden region. After testing the TETF functionals against HF results, we apply them to discuss the temperature dependence of fission barriers and LDM parameters. The latter are evaluated for two different situations: that of an isolated, metastable hot nucleus, and that of a nucleus in thermodynamical equilibrium with a surrounding nucleon gas, such as is met in astrophysical applications.

Some of the more involved formulae and technical details are given in a series of appendices.

\section{Justification of the semiclassical approach based on the microscopical Skyrme-HF formalism}

\subsection{The Skyrme-HF energy density}

We shall recall here the expression for the energy density obtained with Skyrme type forces in the HF approximation. For its derivation we refer to the original article by Vautherin and Brink [3]. We shall use the extended type of Skyrme forces where the density-dependent term contains a variable power of the density (see e.g. ref. [24]). The total HF energy is written in the form

$$
E_{\mathrm{HF}}=\int \mathrm{d}^{3} r\left[\mathscr{E}_{\mathrm{Sky}}(r)+\mathscr{E}_{\mathrm{Coul}}(r)\right]
$$

The nuclear (Skyrme) energy density for even-even nuclei can be expressed in terms of the local nucleon densities $\rho_{\mathrm{q}}(\boldsymbol{r})$, kinetic energy densities $\tau_{\mathrm{q}}(\boldsymbol{r})$ and spin-orbit densities $J_{\mathrm{q}}(\boldsymbol{r})$ defined by $(\mathrm{q}=\mathrm{n}, \mathrm{p}$ for neutrons and protons, respectively)

$$
\begin{aligned}
& \rho_{\mathrm{q}}(\boldsymbol{r})=\sum_{\nu, s}\left|\varphi_{\nu}(r, s, \mathrm{q})\right|^{2} n_{\nu}^{\mathrm{q}}, \\
& \tau_{\mathrm{q}}(\boldsymbol{r})=\sum_{\nu, s}\left|\nabla \varphi_{\nu}(r, s, \mathrm{q})\right|^{2} n_{\nu}^{\mathrm{q}}, \\
& J_{\mathrm{q}}(\boldsymbol{r})=(-\mathrm{i}) \sum_{\nu, s, s^{\prime}} \varphi_{\nu}^{*}\left(r, s^{\prime}, \mathrm{q}\right) \nabla \varphi_{\nu}(r, s, \mathrm{q}) \times\left\langle s^{\prime}|\boldsymbol{\sigma}| s\right\rangle n_{\nu}^{\mathrm{q}},
\end{aligned}
$$


where $\varphi_{\nu}(r, s, q)$ are the single-particle wave functions with orbital and spin quantum numbers $\nu$ and $s$, respectively, and $n_{\nu}^{q}$ are the occupation numbers (equal to 1 or 0 in the pure HF case, or $v_{\nu}^{2}$ if pairing correlations are included in the BCS approximation [25]). Defining the total densities (leaving out their arguments, where it cannot cause confusion) $\rho=\rho_{\mathrm{n}}+\rho_{\mathrm{p}}, \tau=\tau_{\mathrm{n}}+\tau_{\mathrm{p}}$ and $\boldsymbol{J}=J_{\mathrm{n}}+J_{\mathrm{p}}$, the Skyrme energy density takes the explicit form

$$
\begin{aligned}
\mathscr{E}_{\text {Sky }}(\boldsymbol{r})= & \frac{\hbar^{2}}{2 m} \tau+\frac{1}{2} t_{0}\left[\left(1+\frac{1}{2} x_{0}\right) \rho^{2}-\left(x_{0}+\frac{1}{2}\right)\left(\rho_{\mathrm{n}}^{2}+\rho_{\mathrm{p}}^{2}\right)\right] \\
& +\frac{1}{12} t_{3} \rho^{\alpha}\left[\left(1+\frac{1}{2} x_{3}\right) \rho^{2}-\left(x_{3}+\frac{1}{2}\right)\left(\rho_{\mathrm{n}}^{2}+\rho_{\mathrm{p}}^{2}\right)\right]+\frac{1}{4}\left[t_{1}\left(1+\frac{1}{2} x_{1}\right)+t_{2}\left(1+\frac{1}{2} x_{2}\right)\right] \tau \rho \\
& +\frac{1}{4}\left[t_{2}\left(x_{2}+\frac{1}{2}\right)-t_{1}\left(x_{1}+\frac{1}{2}\right)\right]\left(\tau_{\mathrm{n}} \rho_{\mathrm{n}}+\tau_{\mathrm{p}} \rho_{\mathrm{p}}\right)+\frac{1}{16}\left[3 t_{1}\left(1+\frac{1}{2} x_{1}\right)-t_{2}\left(1+\frac{1}{2} x_{2}\right)\right](\nabla \rho)^{2} \\
& -\frac{1}{16}\left[3 t_{1}\left(x_{1}+\frac{1}{2}\right)+t_{2}\left(x_{2}+\frac{1}{2}\right)\right]\left[\left(\nabla \rho_{\mathrm{n}}\right)^{2}+\left(\nabla \rho_{\mathrm{p}}\right)^{2}\right]+\frac{1}{2} W_{0}\left[J \cdot \nabla \rho+J_{\mathrm{n}} \cdot \nabla \rho_{\mathrm{n}}+J_{\mathrm{p}} \cdot \nabla \rho_{\mathrm{p}}\right]
\end{aligned}
$$

The Coulomb energy density is the sum of the direct term and the exchange term, the latter taken in the well-known Slater approximation which has proved sufficiently accurate for all practical purposes [26]:

$$
\mathscr{E}_{\text {Coul }}(r)=e^{2} \rho_{\mathrm{p}}(r) \frac{1}{2} \int \mathrm{d}^{3} r^{\prime} \frac{\rho_{\mathrm{p}}\left(\boldsymbol{r}^{\prime}\right)}{\left|\boldsymbol{r}-\boldsymbol{r}^{\prime}\right|}-\frac{3}{4} e^{2}\left(\frac{3}{\pi}\right)^{1 / 3} \rho_{\mathrm{p}}^{4 / 3}(\boldsymbol{r})
$$

We shall also be using the energy density of Tondeur [27] which is very similar to the Skyrme energy density, although it cannot be derived from a simple two-body force:

$$
\mathscr{E}_{\text {Tond }}(r)=\frac{\hbar^{2}}{2 m} \tau+a \rho^{2}+b \rho^{\gamma}+c \rho^{-1 / 3}\left(\rho_{\mathrm{n}}-\rho_{\mathrm{p}}\right)^{2}+d J \cdot \nabla \rho+\eta(\nabla \rho)^{2}
$$

The HF equations, obtained by varying the wave functions $\varphi_{\nu}^{q}=\varphi_{\nu}(r, s, q)$, take the form

$$
\hat{H}_{\mathrm{HF}}^{\mathrm{q}} \varphi_{\nu}^{\mathrm{q}}=\left[-\nabla \cdot \frac{\hbar^{2}}{2 m_{\mathrm{q}}^{*}(r)} \nabla+V_{\mathrm{q}}(\boldsymbol{r})-\mathrm{i} \boldsymbol{W}_{\mathrm{q}}(r) \cdot(\nabla \times \boldsymbol{\sigma})\right] \varphi_{\nu}^{\mathrm{q}}=\varepsilon_{\nu}^{\mathrm{q}} \varphi_{\nu}^{\mathrm{q}} .
$$

The local potentials $V_{\mathrm{q}}(\boldsymbol{r})$, effective masses $m_{\mathrm{q}}^{*}(\boldsymbol{r})$ and spin-orbit potentials $\boldsymbol{W}_{\mathrm{q}}(\boldsymbol{r})$ are given by the relations

$$
\begin{aligned}
& V_{\mathrm{q}}(\boldsymbol{r})=\delta \mathscr{E}(\boldsymbol{r}) / \delta \rho_{\mathrm{q}}(\boldsymbol{r}) \equiv \partial \mathscr{E} / \partial \rho_{\mathrm{q}}-\nabla \cdot \partial \mathscr{E} / \partial\left(\nabla \rho_{\mathrm{q}}\right)+\Delta \partial \mathscr{E} / \partial\left(\Delta \rho_{\mathrm{q}}\right) \\
& \hbar^{2} / 2 m_{\mathrm{q}}^{*}(\boldsymbol{r})=\partial \mathscr{E}(\boldsymbol{r}) / \partial \tau_{\mathrm{q}}(\boldsymbol{r}) \\
& \boldsymbol{W}_{\mathrm{q}}(\boldsymbol{r})=\partial \mathscr{E}(\boldsymbol{r}) / \partial J_{\mathrm{q}}(\boldsymbol{r})
\end{aligned}
$$

where $\mathscr{E}(r)$ is the sum of the nuclear (i.e. Skyrme or Tondeur) and the Coulomb energy density. (Note that the Tondeur energy density eq. (2.7) leads to constant nucleon masses: $m_{\mathrm{q}}^{*}(r)=m$.)

Usually, the force parameters are determined by fits of experimental ground-state properties of a series of (mostly spherical) nuclei. However, most of them are related to each other, and restricted in their range of values, by imposing the more or less well-established saturation properties of infinite nuclear matter. It may be worth writing down the corresponding expressions. 
For symmetric infinite nuclear matter (without Coulomb interaction), we put $\rho_{\mathrm{n}}=\rho_{\mathrm{p}}=\frac{1}{2} \rho=$ const. and obtain from eq. (2.5) for the binding energy per nucleon

$$
(E / A)_{\infty}=e_{\infty}(\rho)=k \rho^{2 / 3}(1+\beta \rho)+\frac{3}{8} t_{0} \rho+\frac{1}{16} t_{3} \rho^{1+\alpha},
$$

where we have put

$$
k=\frac{\hbar^{2}}{2 m} \frac{3}{5}\left(\frac{3 \pi^{2}}{2}\right)^{2 / 3} \simeq 75.0 \mathrm{MeV} \mathrm{fm}{ }^{2}
$$

and

$$
\beta=\frac{2 m}{\hbar^{2}} \frac{1}{4}\left[\frac{1}{4}\left(3 t_{1}+5 t_{2}\right)+t_{2} x_{2}\right] .
$$

The saturation density $\rho_{\infty}$ is fixed by the condition

$$
\left.\frac{\mathrm{d} e_{\infty}(\rho)}{\mathrm{d} \rho}\right|_{\rho_{\infty}}=0
$$

The infinite nuclear matter incompressibility is

$$
\begin{aligned}
K_{\infty} & =\left.9 \rho_{\infty}^{2} \frac{\mathrm{d}^{2} e_{\infty}(\rho)}{\mathrm{d} \rho^{2}}\right|_{\rho_{\infty}} \\
& =-2 k \rho_{\infty}^{2 / 3}+10 k \beta \rho_{\infty}^{5 / 3}+9 \frac{\alpha(\alpha+1)}{16} t_{3} \rho_{\infty}^{1+\alpha},
\end{aligned}
$$

and the (isoscalar) effective nucleon mass $m_{\infty}^{*}$ is given by

$$
m / m_{\infty}^{*}=1+\beta \rho_{\infty} .
$$

The above eqs. (2.12)-(2.17) hold also for the Tondeur functional eq. (2.7) if one puts:

$$
t_{0}=3 a, \quad t_{1}=-t_{2}, \quad x_{2}=-\frac{1}{2}, \quad t_{3}=16 b, \quad \alpha=\gamma-2, \quad \beta=0 .
$$

For asymmetric nuclear matter with neutron and proton densities $\rho_{0 \mathrm{n}}$ and $\rho_{0 \mathrm{p}}$, respectively, one usually defines the volume asymmetry energy by

$$
a_{\mathrm{as}}=J=\frac{1}{2 \rho_{0}}\left\{\frac{\mathrm{d}^{2}}{\mathrm{~d} \delta^{2}}\left[\rho_{0} e_{\mathrm{co}}\left(\rho_{0 \mathrm{n}}, \rho_{0 \mathrm{p}}\right)\right]\right\}_{\delta=0},
$$

where the asymmetry parameter $\delta$ is given by [19]

$$
\delta=\left(\rho_{0 \mathrm{n}}-\rho_{0 \mathrm{p}}\right) / \rho_{0}, \quad \rho_{0}=\rho_{0 \mathrm{n}}+\rho_{0 \mathrm{p}}
$$


Note that the saturation density $\rho_{0}$ of asymmetric matter in general is different from $\rho_{\infty}$ (see section 5 for details). For Skyrme forces one finds

$$
J=\frac{5}{9} k \rho_{0}^{2 / 3}+\frac{5}{3} \frac{2 m}{\hbar^{2}} k\left[\frac{1}{6} t_{2}\left(1+\frac{5}{4} x_{2}\right)-\frac{1}{8} t_{1} x_{1}\right] \rho_{0}^{5 / 3}-\frac{1}{24} t_{3}\left(\frac{1}{2}+x_{3}\right) \rho_{0}^{1+\alpha}-\frac{1}{4} t_{0}\left(\frac{1}{2}+x_{0}\right) \rho_{0},
$$

and for the Tondeur functional

$$
J=\left(\frac{5}{9} k+c\right) \rho_{0}^{2 / 3}
$$

Imposing reasonable values for the volume energy $a_{v}^{\infty}=e_{\infty}\left(\rho_{\infty}\right)$, for $K_{\infty}, \rho_{\infty}, m_{\infty}^{*}$ and for $J$ through the above relations, the choice of the force parameters is greatly restricted, although still innumerable parameter sets can be found in the literature (see, e.g. ref. [1]). The parameter $\alpha$ of the densitydependent term in the Skyrme functional eq. (2.5) is rather strongly restricted by the values of $K_{\infty}$ and $m_{\infty}^{*}$. In fact, if values in the ranges

$$
\begin{aligned}
& 210 \mathrm{MeV} \leq K_{\infty} \leq 240 \mathrm{MeV} \\
& 0.7 \leq m_{\infty}^{*} / m \leq 0.8
\end{aligned}
$$

are imposed, as they are required in order to fit the giant monopole and quadrupole resonances by RPA calculations $[28,29]$, one finds that $\alpha$ must be of the order

$$
\frac{1}{6} \leqslant \alpha \leqslant \frac{1}{3} \text {. }
$$

This rules out a whole series of Skyrme force parametrizations, labeled S I, S II [3] and S III-S VI [30], which have $\alpha=1$ and $K_{\infty}$ values of the order of $340-400 \mathrm{MeV}$. We shall nevertheless be using the S III force, too, in this paper, since it has been the favourite Skyrme force for a long time due to its particularly nice fits of nuclear binding energies and various spectroscopic data $[1,30]$.

In table 1 we list the Skyrme parameter sets which will be encountered in this paper. The parameters of the Tondeur energy density eq. (2.7) may be found in ref. [27].

Table 1

Parameters of the Skyrme forces used in this article. References: SIII [30], Ska [33], SkM

[34], RATP [120]. The SkM* force is discussed in section 4.4 (see also ref. [7])

\begin{tabular}{lrrrrr}
\hline Force & \multicolumn{1}{l}{ SIII } & \multicolumn{1}{c}{ Ska } & \multicolumn{1}{c}{ SkM } & \multicolumn{1}{c}{ SkM } & \multicolumn{1}{c}{ RATP } \\
\hline$t_{0}\left(\mathrm{MeV} \mathrm{fm}^{3}\right)$ & -1128.75 & -1602.78 & -2645.00 & -2645.00 & -2160.00 \\
$t_{1}\left(\mathrm{MeV} \mathrm{fm}^{5}\right)$ & 395.00 & 570.88 & 385.00 & 410.00 & 513.00 \\
$t_{2}\left(\mathrm{MeV} \mathrm{fm}^{5}\right)$ & -95.00 & -67.70 & -120.00 & -135.00 & 121.00 \\
$t_{3}\left(\mathrm{MeV} \mathrm{fm}^{3+3 \alpha}\right)$ & 14000.00 & 8000.00 & 15595.00 & 15595.00 & 11600.00 \\
$x_{0}$ & 0.45 & -0.02 & 0.09 & 0.09 & 0.418 \\
$x_{1}$ & 0.00 & 0.00 & 0.00 & 0.00 & -0.36 \\
$x_{2}$ & 0.00 & 0.00 & 0.00 & 0.00 & -2.29 \\
$x_{3}$ & 1.00 & -0.286 & 0.00 & 0.00 & 0.586 \\
$\alpha$ & 1.00 & $1 / 3$ & $1 / 6$ & $1 / 6$ & $1 / 5$ \\
$W_{0}\left(\mathrm{MeV} \mathrm{fm}^{5}\right)$ & 120.00 & 125.00 & 130.00 & 130.00 & 120.00 \\
\hline
\end{tabular}


In table 2 we have listed the nuclear matter properties of all these forces, including the radius constant $r_{0}=\left(4 \pi \rho_{\infty} / 3\right)^{-1 / 3}$ and the Fermi momentum $k_{\mathrm{F}}=\left(3 \pi^{2} \rho_{\infty} / 2\right)^{1 / 3}$, as well as the constants $L, M$ and $\dddot{a}_{v}$, which will be discussed in connection with the droplet model in section 5 .

Having imposed "reasonable" nuclear matter properties alone guarantees, of course, in no way that a force will have good surface properties of finite nuclei, which then are adjusted by actual HF calculations and fits to experimental data. Even more it must be considered a great success that good fits to many data were obtained, considering the fact that the nuclear matter properties fix already five combinations of the typically 7-8 Skyrme parameters (in most cases, $x_{1}=x_{2}=0$ and pre-fixed values of $x_{3}$ were used). For detailed comparisons of HF $(+B C S)$ results to experimental data, we can only refer here to the abundant literature $[1,28-32]$.

Table 2

Properties of infinite nuclear matter for various Skyrme forces and for the Tondeur energy density [27]

\begin{tabular}{lcccccc}
\hline Force & SIII & \multicolumn{1}{l}{ Ska } & \multicolumn{1}{l}{ SkM } & \multicolumn{1}{l}{ SkM* } & To 78 & RATP \\
\hline$\rho_{\infty}\left(\mathrm{fm}^{-3}\right)$ & 0.1453 & 0.1554 & 0.1603 & 0.1603 & 0.1590 & 0.1599 \\
$a_{v}^{\infty}(\mathrm{MeV})$ & -15.857 & -15.997 & -15.776 & -15.776 & -15.978 & -16.052 \\
$K_{\infty}(\mathrm{MeV})$ & 355.4 & 263.2 & 216.7 & 216.7 & 235.8 & 239.6 \\
$J(\mathrm{MeV})$ & 28.16 & 32.91 & 30.75 & 30.03 & 32.12 & 29.26 \\
$m_{\infty}^{*} / m$ & 0.76 & 0.61 & 0.79 & 0.79 & 1.00 & 0.67 \\
$r_{0}(\mathrm{fm})$ & 1.180 & 1.154 & 1.142 & 1.142 & 1.145 & 1.143 \\
$k_{\mathbf{F}}\left(\mathrm{fm}^{-1}\right)$ & 1.291 & 1.320 & 1.334 & 1.334 & 1.330 & 1.333 \\
$L(\mathrm{MeV})$ & 9.9 & 75.3 & 49.3 & 45.8 & 64.2 & 32.4 \\
$M(\mathrm{MeV})$ & 1.66 & 2.26 & 1.82 & 1.88 & 0.91 & 1.90 \\
$\ddot{a}_{\mathbf{v}}(\mathrm{MeV})$ & 3.8 & -11.3 & -14.3 & -14.3 & -14.2 & -13.0 \\
\hline
\end{tabular}

\subsection{Separation of shell effects}

The direct application of the Skyrme energy functional eq. (2.5) - or of any similar functional - to the energy density method is handicapped by the presence of the kinetic energy and spin-orbit densities $\tau_{\mathrm{q}}(\boldsymbol{r})$ and $\boldsymbol{J}_{\mathrm{q}}(\boldsymbol{r})$. In principle, we know from the Hohenberg-Kohn theorem [14] that there exist unique functionals $\tau[\rho]$ and $J[\rho]$ which allow to express these densities in terms of the local nucleon densities $\rho_{\mathrm{q}}(r)$. However we do not know these functionals and there is little chance to determine them exactly. They certainly must be nonlocal, since the shell effects contained in $\tau_{\mathrm{q}}(\boldsymbol{r})$ and $\boldsymbol{J}_{\mathrm{q}}(\boldsymbol{r})$ are not local, but global properties of the nucleus $[35,36]$.

This problem can be overcome by averaging out the shell effects and expressing the average part of the energy by a functional of the average densities $\tilde{\rho}_{\mathrm{q}}(r)$. This can be justified by means of Strutinsky's energy-averaging method [20] which, in fact, allows to decompose the exact HF energy in a rather unique way into an average and a fluctuating ("shell-correction") part (see also refs. [17, 37]):

$$
E_{\mathrm{HF}} \simeq \tilde{E}_{\mathrm{HF}}+\delta_{1} E_{\mathrm{n}}+\delta_{1} E_{\mathrm{p}} .
$$

Hereby the average energy $\tilde{E}_{\mathrm{HF}}$ is practically calculated in the same way as the exact energy $E_{\mathrm{HF}}$ through eqs. (2.1)-(2.6), but replacing the quantum mechanical densities eqs. (2.2)-(2.4) by the averaged densities obtained by means of the Strutinsky-averaging occupation numbers $\tilde{n}_{\nu}^{\mathrm{q}}[37,38]$, i.e.

$$
\tilde{\rho}_{\mathrm{q}}(\boldsymbol{r})=\sum_{\nu, s}\left|\varphi_{\nu}(\boldsymbol{r}, s, \mathrm{q})\right|^{2} \tilde{n}_{\nu}^{\mathrm{q}}
$$


etc. The shell-correction energy $\delta_{1} E_{\mathrm{q}}$ in eq. (2.24) is defined by

$$
\delta_{1} E_{\mathrm{q}}=\sum_{\nu} \hat{\varepsilon}_{\nu}^{\mathrm{q}}\left(n_{\nu}^{\mathrm{q}}-\tilde{n}_{\nu}^{\mathrm{q}}\right)
$$

where $\hat{\varepsilon}_{\nu}^{\mathrm{q}}$ are the eigenvalues of the average HF Hamiltonians $\tilde{H}_{\mathrm{HF}}^{\mathrm{q}}$ defined through eqs. (2.8)-(2.11) in terms of the averaged densities, i.e.

$$
\tilde{H}_{\mathrm{HF}}^{\mathrm{q}} \hat{\varphi}_{\nu}^{\mathrm{q}}=\hat{H}_{\mathrm{HF}}\left[\tilde{\rho}_{\mathbf{q}}, \tilde{\tau}_{\mathrm{q}}, \tilde{J}_{\mathrm{q}}\right] \hat{\varphi}_{\nu}^{\mathrm{q}}=\hat{\varepsilon}_{\nu}^{\mathrm{q}} \hat{\varphi}_{\nu}^{\mathrm{q}} .
$$

Formally, eq. (2.24) just represents the lowest two terms of a Taylor expansion of the HF energy around the average parts of the densities. (See ref. [17] for a discussion and further literature on this subject.) In extended numerical calculations [17] it has been checked that the missing higher order terms in eq. (2.24) are negligible for all practical purposes. In particular if the averaging by means of the $\tilde{n}_{\nu}^{\mathrm{q}}$ is done selfconsistently (see also the next subsection), the two sides of eq. (2.24) are equal to within less than $\sim 0.5 \mathrm{MeV}$ even in heavy, strongly deformed nuclei (corresponding to an accuracy of better than $10^{-3}$ ).

Two important conclusions could be drawn from the numerical results reported in ref. [17]:

1) The averaged HF energy $\tilde{E}_{\mathrm{HF}}$ has all the properties of a LDM type, semiclassical energy.

2) The selfconsistency is only important for the average quantities $\left(\tilde{E}_{\mathrm{HF}}, \tilde{H}_{\mathrm{HF}}, \tilde{\rho}_{\mathrm{q}}\right.$, etc.); the shell effects can, in fact, be added perturbatively.

This provides us with a strong motivation to replace the above-sketched microscopical selfconsistent calculation of $\tilde{E}_{\mathrm{HF}}$ by a semiclassical calculation. For its realization, it was important to quantitatively secure the equivalence of the Strutinsky-averaging procedure with a semiclassical expansion of the energy, as will be discussed in the following subsection.

\subsection{Strutinsky-averaging as a microscopical link to the ETF model}

Strutinsky [20] and Tyapin [39] surmized that the numerically Strutinsky-averaged energies not only correspond to those obtained in the Fermi gas theory, but that they contain also inhomogeneity corrections such as they are obtained in the so-called extended Thomas-Fermi (ETF) model [40, 41].

Bhaduri and Ross [6] proposed to calculate the average energy of nucleons in various model potentials by employing a $\hbar$-expansion of the partition function, which actually had been developed long ago by Wigner [42] and Kirkwood [43], and demonstrated the closeness of their results to those of a numerical Strutinsky averaging. (We shall discuss the Wigner-Kirkwood expansion and the ETF relations derived from it in detail in section 3.)

For harmonic oscillator potentials, the exact equivalence of the Strutinsky-averaging method and the semiclassical $\hbar$-expansion was proved analytically $[38,44]$. For realistic, deformed Woods-Saxon type potentials including spin-orbit fields, the two methods were shown numerically [45] to yield identical energies to within $\sim 1-1.5 \mathrm{MeV}$ (of several $\mathrm{GeV}$ ), which is roughly the uncertainty in either method.

It is thus well established that - at least as energies are concerned and with the numerical accuracy practically required - the microscopical Strutinsky-averaging procedure is equivalent to a semiclassical $\hbar$-expansion. Therefore it seems natural to use the ETF functionals $\tau[\rho]$ and $J[\rho]$ obtained from the same $\hbar$-expansion (see next section) in order to calculate the average $\mathrm{HF}$ energy $\tilde{E}_{\mathrm{HF}}$ in a semiclassical, and thus much more economical way.

That the energy $\tilde{E}_{\mathrm{HF}}-$ which was obtained microscopically in ref. [17], as explained in section 
2.2 - can be expressed as a functional of the average densities $\tilde{\rho}_{\mathbf{q}}(\boldsymbol{r})$ eq. (2.25) is again a consequence of the Hohenberg-Kohn theorem. The iterative inclusion of the Strutinsky occupation numbers $\tilde{n}_{\nu}^{q}$ in the HF cycle has, in fact, been formulated in a strictly variational way in ref. [17], including a proper constraint in the energy to be made stationary (and found to be minimized in actual calculations).

The Hohenberg-Kohn theorem [14] applies therefore to this variational averaged system as well as it applies to any variational system of fermions interacting through a 2-body force. (For a recent discussion of this theorem and its applications, see ref. [46].)

\subsection{Summary}

Let us summarize at this point the main steps of the derivation and justification of our semiclassical variational method.

1) HF calculations with effective Skyrme interactions allow to calculate a vast amount of nuclear ground-state properties, deformation energies and (with RPA) giant resonances to a satisfactory degree.

2) The HF energy can be split, by means of the Strutinsky-averaging procedure, in a selfconsistent average part $\tilde{E}_{\mathrm{HF}}$ and a shell-correction part, see eq. (2.24).

3) The averaged energy $\tilde{E}_{\mathrm{HF}}$ and corresponding selfconsistent average densities $\tilde{\rho}_{\mathrm{q}}(\boldsymbol{r})$ can be obtained in a strictly variational way (ref. [17]). Therefore, by virtue of the Hohenberg-Kohn theorem, $\tilde{E}_{\mathrm{HF}}$ and thus $\tilde{\tau}_{\mathrm{q}}(\boldsymbol{r})$ and $\tilde{J}_{\mathrm{q}}(\boldsymbol{r})$ are unique functionals of $\rho_{\mathrm{q}}(\boldsymbol{r})$.

4) The Strutinsky-averaging method is practically equivalent to a semiclassical $\hbar$-expansion of the energy (ref. [45]).

5) Combining 3 ) and 4) allows to express $\tilde{E}_{\mathrm{HF}}$.in terms of $\tilde{\rho}_{\mathrm{q}}(\boldsymbol{r})$ only by means of the ETF functionals $\tau[\rho]$ and $J[\rho]$ obtained in the same $\hbar$-expansion, and to perform semiclassical density variational calculations in order to optimize $\tilde{\rho}_{\mathrm{q}}(r)$.

6) After selfconsistency has been reached for $\tilde{E}_{\mathrm{HF}}$ and $\tilde{\rho}_{\mathrm{q}}(\boldsymbol{r})$, the average mean fields eqs. (2.9)(2.11) can be used to calculate the shell-correction energies $\delta_{1} E_{\mathrm{q}}(2.26)$ by solving once the Schrödinger equation (2.27). Adding $\delta_{1} E_{\mathrm{q}}$ to $\tilde{E}_{\mathrm{HF}}$, thus incorporating the shell effects perturbatively, allows to recover the (exact) HF energy with sufficient accuracy (see eq. (2.24) and ref. [17]).

\section{The extended Thomas-Fermi model}

\subsection{The Wigner-Kirkwood expansion}

We shall in the following sketch the semiclassical $\hbar$-expansion developed by Wigner [42] and Kirkwood [43], which provides a convenient tool to derive the ETF functionals $\tau[\rho]$ and $J[\rho]$ which we are interested in. For the sake of a simple notation, we shall presently restrict ourselves to the case of $N$ nucleons (one kind only) in a given local (HF) potential $V(r)$. Let $\varphi_{\nu}$ and $\varepsilon_{\nu}$ be the eigenfunctions and eigenvalues of the corresponding Schrödinger equation:

$$
\hat{H} \varphi_{\nu}=[\hat{T}+V(r)] \varphi_{\nu}=\varepsilon_{\nu} \varphi_{\nu} .
$$

Next we define the Bloch density matrix

$$
C\left(\boldsymbol{r}, \boldsymbol{r}^{\prime} ; \beta\right)=\sum_{\nu} \varphi_{\nu}^{*}\left(\boldsymbol{r}^{\prime}\right) \varphi_{\nu}(\boldsymbol{r}) \exp \left(-\beta \varepsilon_{\nu}\right)
$$


where the sum goes over the complete spectrum (including an integral over the continuum, if present). From $C$, we obtain by an inverse Laplace transform the usual density matrix (see also appendix B)

$$
\rho\left(r, r^{\prime}\right)=\mathscr{L}_{\lambda}^{-1}\left[\frac{1}{\beta} C\left(r, r^{\prime} ; \beta\right)\right]=\frac{1}{2 \pi \mathrm{i}} \int_{c-\mathrm{i} \infty}^{c+\mathrm{i} \infty} \mathrm{d} \beta \mathrm{e}^{\lambda \beta} \frac{1}{\beta} C\left(r, r^{\prime} ; \beta\right)
$$

from which, in turn, the local densities $\rho(r)$ and $\tau(r)$ can be determined

$$
\begin{aligned}
& \rho(r)=\sum_{\nu=1}^{N}\left|\varphi_{\nu}(r)\right|^{2}=\rho(r, r) \\
& \tau(r)=\sum_{\nu=1}^{N}\left|\nabla \varphi_{\nu}(r)\right|^{2}=\left.\nabla_{r} \cdot \nabla_{r^{\prime}} \rho\left(r, r^{\prime}\right)\right|_{r=r^{\prime}}
\end{aligned}
$$

In eq. (3.3), $\lambda$ is the Fermi energy which is fixed by the particle number conservation

$$
\int \rho(r) \mathrm{d}^{3} r=N
$$

The idea of Wigner and Kirkwood was to expand $C\left(r, r^{\prime} ; \beta\right)$ around its value obtained in the Thomas-Fermi approximation:

$$
C_{\mathrm{TF}}\left(\boldsymbol{r}, \boldsymbol{r}^{\prime} ; \boldsymbol{\beta}\right)=\left(\frac{m}{2 \pi \hbar^{2} \beta}\right)^{3 / 2} \exp \left\{-\boldsymbol{\beta} V\left(\frac{\boldsymbol{r}+\boldsymbol{r}^{\prime}}{2}\right)\right\} \exp \left\{-\frac{m}{2 \hbar^{2} \cdot \boldsymbol{\beta}}\left(\boldsymbol{r}-\boldsymbol{r}^{\prime}\right)^{2}\right\}
$$

One makes the ansatz

$$
C\left(\boldsymbol{r}, \boldsymbol{r}^{\prime} ; \beta\right)=C_{\mathrm{TF}}\left(\boldsymbol{r}, \boldsymbol{r}^{\prime} ; \beta\right)\left\{1+\hbar_{\chi_{1}}+\hbar^{2} \chi_{2}+\cdots\right\}
$$

thus expanding the ratio of the exact to the TF Bloch function in powers of $\hbar$. The $\chi_{n}$ are functions of $r, r^{\prime}$ and $\beta$ which contain combinations of $n$ gradients acting on $V(r)$. Uhlenbeck and Beth [47] worked out a recursive scheme to obtain the $\chi_{n}$ successively (see also ref. [48]). By Laplace inverting the series eq. (3.8) back term by term, one obtains an expansion of the density matrix eq. (3.3) and thus of $\rho(r)$ and $\tau(r)$, to which only even powers of $\hbar$ (i.e. $\chi_{n}$ with even $n$ ) contribute. We quote here the results up to order $\hbar^{2}$

$$
\begin{aligned}
& \rho_{\mathrm{ETF}}(r)=\frac{1}{3 \pi^{2}}\left(\frac{2 m}{\hbar^{2}}\right)^{3 / 2}(\lambda-V(r))^{3 / 2} \theta(\lambda-V(r))\left\{1-\frac{1}{8} \frac{\hbar^{2}}{2 m}\left[\Delta V(\lambda-V)^{-2}+\frac{1}{4}(\nabla V)^{2}(\lambda-V)^{-3}\right]\right\}, \\
& \tau_{\mathrm{ETF}}(r)=\frac{1}{5 \pi^{2}}\left(\frac{2 m}{\hbar^{2}}\right)^{5 / 2}(\lambda-V(r))^{5 / 2} \theta(\lambda-V(r))\left\{1-\frac{5}{8} \frac{\hbar^{2}}{2 m}\left[\frac{5}{3} \Delta V(\lambda-V)^{-2}-\frac{3}{4}(\nabla V)^{2}(\lambda-V)^{-3}\right]\right\} .
\end{aligned}
$$


In the lowest order terms we recognize the TF expressions; the $\hbar^{2}$-corrections lead to the well-known divergencies at the classical turning points $r_{\lambda}$ given by $\lambda=V\left(r_{\lambda}\right)$. (Due to the step functions, both densities are identically zero outside the classically allowed region.)

In spite of their turning point divergencies, the densities eqs. (3.9), (3.10) can be shown [48] to lead to finite energies and particle numbers, even if the $\hbar^{4}$-terms are included. This shows that the ETF densities are rather to be understood as distributions with well-defined integrals and moments (see also ref. [49]). The energies so obtained form a rapidly converging asymptotic series

$$
E_{\mathrm{ETF}}=\int \mathrm{d}^{3} r\left[V(r) \rho_{\mathrm{ETF}}(r)+\frac{\hbar^{2}}{2 m} \tau_{\mathrm{ETF}}(r)\right]=E_{\mathrm{TF}}+E_{2}+E_{4}+\cdots
$$

The sum of the first three terms (i.e. up to order $\hbar^{4}$ ) converges typically to within $\sim 1 \mathrm{MeV}$ and agrees, as mentioned in section 2.3 above, with the energy obtained by Strutinsky averaging:

$$
E_{\mathrm{ETF}} \simeq \tilde{E}_{\mathrm{Str}}=\sum_{\nu} \varepsilon_{\nu} \tilde{n}_{\nu}
$$

We shall not discuss here the technicalities of including effective mass and spin-orbit contributions, which can be done starting from a Hamiltonian of Skyrme type eq. (2.8); they can be found in the literature $[45,48]$.

Before coming to the construction of the ETF density functionals, we mention that a way of removing the turning point divergencies in $\rho_{\mathrm{ETF}}(r)$ and $\tau_{\mathrm{ETF}}(r)$ by partially resumming the WignerKirkwood series eq. (3.8) has been proposed recently by Bhaduri [50] and further developed [51, 52]. The so obtained densities $\rho$ and $\tau$ can be iterated directly to obtain selfconsistency [53] without use of any functional $\tau[\rho]$. This provides another semiclassical variational method which will be published elsewhere [54].

\subsection{The ETF functionals $\tau[\rho]$ and $J[\rho]$}

\subsubsection{Local case}

From eqs. (3.9) and (3.10) it is possible to eliminate algebraically the Fermi energy $\lambda$, the potential $V(r)$ and its derivatives, hereby consistently retaining all terms of order $\hbar^{2}$ and neglecting those of higher orders in $\hbar$. The result is (for one kind of nucleons)

$$
\tau[\rho]=\tau_{\mathrm{TF}}[\rho]+\tau_{2}[\rho]
$$

with the well-known Thomas-Fermi relation

$$
\tau_{\mathrm{TF}}[\rho]=\kappa \rho^{5 / 3}, \quad \kappa=\frac{3}{5}\left(3 \pi^{2}\right)^{2 / 3}
$$

and the second order gradient correction

$$
\tau_{2}[\rho]=\frac{1}{36} \frac{(\nabla \rho)^{2}}{\rho}+\frac{1}{3} \Delta \rho
$$


The first term in $\tau_{2}[\rho]$ is the so-called Weizsäcker correction, named after the author of ref. [8] who derived it in a somewhat ad hoc manner and obtained it with a 9 times larger coefficient. This coefficient has subsequently given rise to a lot of discussion (see ref. [10] for the literature). By now it is clear that various alternative semiclassical expansion procedures $[16,39,40]$ lead to exactly the same relations, eqs. (3.9), (3.10) and (3.15). (For a recent review in which these alternative expansions are discussed and related, see ref. [55].) The coefficient $1 / 36$ of the Weizsäcker term is thus well established in the framework of semiclassical expansions (and for smooth potentials $V(r)$ ). The second term in eq. (3.15) does not contribute to the integrated kinetic energy and has therefore often been ignored; it does however contribute to the total Skyrme energy through the terms containing $\tau \rho$ (see eq. (2.5)).

Going up to order $\hbar^{4}$ in the expansion of $\rho_{\mathrm{ETF}}$ and $\tau_{\mathrm{ETF}}$ and proceeding in the same way, one obtains the next correction $\tau_{4}[\rho]$ to the functional, containing up to the fourth derivative of $\rho$. The somewhat lengthy expression for $\tau_{4}[\rho]$ is given in appendix $\mathrm{A}$. When integrating over the whole space, the fourth and third derivatives of $\rho$ can be eliminated by partial integration, and the expression simplifies to

$$
\int \tau_{4}[\rho] \mathrm{d}^{3} r=\frac{1}{6480}\left(3 \pi^{2}\right)^{-2 / 3} \int \rho^{1 / 3}\left[8\left(\frac{\nabla \rho}{\rho}\right)^{4}-27\left(\frac{\nabla \rho}{\rho}\right)^{2} \frac{\Delta \rho}{\rho}+24\left(\frac{\Delta \rho}{\rho}\right)^{2}\right] \mathrm{d}^{3} r .
$$

Similarly, one obtains

$$
\int \rho \tau_{4}[\rho] \mathrm{d}^{3} r=\frac{1}{3240}\left(3 \pi^{2}\right)^{-2 / 3} \int \rho^{4 / 3}\left[-7\left(\frac{\nabla \rho}{\rho}\right)^{4}-3\left(\frac{\nabla \rho}{\rho}\right)^{2} \frac{\Delta \rho}{\rho}+30\left(\frac{\Delta \rho}{\rho}\right)^{2}\right] \mathrm{d}^{3} r .
$$

This procedure can in principle be continued ad libitum, including higher and higher gradient corrections. However, the terms $\tau_{n}[\rho]$ with $n \geq 6$ diverge for densities which decay exponentially in the tail region. Therefore, the terms up to fourth order must be considered as the converging part of an asymptotic series for $\tau[\rho]$; we shall denote this part by $\tau_{\mathrm{ETF}}[\rho]$ :

$$
\tau_{\mathrm{ETF}}[\rho]=\tau_{\mathrm{TF}}[\rho]+\tau_{2}[\rho]+\tau_{4}[\rho]
$$

The above derivation of the functional $\tau_{\mathrm{ETF}}[\rho]$ is strictly speaking not allowed at the classical turning points, where $\rho_{\mathrm{ETF}}(r)$ and $\tau_{\mathrm{ETF}}(r)$ are singular; outside the classically allowed region the derivation is not possible since the ETF densities are identically zero. However, using an extension of the ETF model to finite temperatures $T$, it is possible to show that $\tau_{\mathrm{ETF}}[\rho]$ is strictly valid in the limit $T \rightarrow 0$ also at and outside the classical turning points. This shall be discussed in detail in section 6.2 below.

The functional $\tau_{\text {ETF }}[\rho]$ given by eqs. (3.14)-(3.18) has been tested numerically with the help of microscopically Strutinsky-averaged densities $\tilde{\tau}(\boldsymbol{r})$ and $\tilde{\rho}(\boldsymbol{r})$, defined as in eq. (2.25), for different spherical and deformed potentials $[15,56]$. The results of these tests may be summarized as follows (for a more detailed discussion, see ref. [56]):

1) The functional $\tau_{\mathrm{ETF}}[\rho]$ reproduces the total Strutinsky-averaged kinetic energy within less than $\sim 1.5 \mathrm{MeV}$, corresponding to a few parts in $10^{4}$ for heavy nuclei. This holds independently of the radial shape of the potential, of its deformation and of the particle number, as it should be expected from the Hohenberg-Kohn theorem.

2) The functional also reproduces the integral $G \int \rho \tau \mathrm{d}^{3} r$, as it occurs in the Skyrme energy, within less than $1 \mathrm{MeV}$ (using realistic Skyrme parameters to determine $G$ ).

3) The terms due to $\tau_{4}[\rho]$ are essential for obtaining the correct deformation energies, in particular the fission barriers, as will be demonstrated in section 4.3 below. 


\subsubsection{Nonlocal case}

For velocity-dependent Skyrme forces, one has to generalize the functional $\tau_{\mathrm{ETF}}[\rho]$, since it receives explicit contributions from the nonlocal parts of the HF potential. Rewriting the Skyrme-HF Hamiltonian (see eq. (2.8)) in the form

$$
\hat{H}_{\text {Sky }}=-\frac{\hbar^{2}}{2 m} \nabla \cdot f(\boldsymbol{r}) \nabla+V(\boldsymbol{r})-\mathrm{i} \boldsymbol{W}(\boldsymbol{r}) \cdot(\nabla \times \boldsymbol{\sigma}),
$$

where $f(r)=m / m^{*}(r)$, the Wigner-Kirkwood expansion eq. (3.8) can be readily obtained. (The Bloch density $C$ is in this case a $2 \times 2$ matrix, the $\chi_{n}$ with $n \geq 1$ containing the Pauli matrices $\sigma_{i}$.) The second order contribution to the kinetic energy density functional then becomes [15]

$$
\tau_{2}[\rho]=\frac{1}{36} \frac{(\nabla \rho)^{2}}{\rho}+\frac{1}{3} \Delta \rho+\frac{1}{6} \frac{(\nabla \rho \cdot \nabla f)}{f}+\frac{1}{6} \rho \frac{\Delta f}{f}-\frac{1}{12} \rho\left(\frac{\nabla f}{f}\right)^{2}+\frac{1}{2}\left(\frac{2 m}{\hbar^{2}}\right)^{2} \rho\left(\frac{W}{f}\right)^{2} .
$$

The spin-orbit density only gets contributions from the $\hbar^{2}$ and higher terms. The lowest order expression is [15]

$$
J_{2}[\rho]=-\left(\frac{2 m}{\hbar^{2}}\right) \frac{1}{f} \rho W=-\left(\frac{2 m^{*}}{\hbar^{2}}\right) \rho W .
$$

(A semiclassical spin-orbit correction equivalent to eq. (3.21) for $m=m^{*}$ has been derived earlier by Stocker et al. [57].)

Carrying through the expansion to fourth order with effective mass and spin-orbit is extremely tedious. It has been carried through with an algebraic computer code by Grammaticos and Voros [16]; we refer to their papers for the explicit expressions for $\tau_{4}[\rho]$ and $J_{4}[\rho]$. In appendix A we give the relevant integrated expressions which again, after suitable partial integrations, contain only first and second derivatives of the densities (cf. eqs. (3.16), (3.17)).

Note that for Skyrme forces $f(r)=1+\beta \rho(r)$ and $W(r)$ is proportional to $\nabla \rho(r)$, so that the functionals $\tau[\rho]$ and $J[\rho]$ ultimately only contain the density $\rho$ and its gradients. We also recall to the reader that the equations in this section hold for either proton or neutron densities and not for the total densities $\tau=\tau_{\mathrm{n}}+\tau_{\mathrm{p}}$ and $\rho=\rho_{\mathrm{n}}+\rho_{\mathrm{p}}$, which explains the difference of a factor $2^{2 / 3}$ between $\kappa$ (eq. (3.14)) and $k$ (eq. (2.13)).

\subsection{Density variation method and discussion of the ETF Euler equations}

Inserting the functionals $\tau_{\mathrm{ETF}}[\rho]$ and $\boldsymbol{J}_{\mathrm{ETF}}[\rho]$ in the expressions for the Skyrme or Tondeur energy density eq. (2.5) or (2.7), respectively, and making use of the variational definitions of $f_{\mathrm{q}}=m / m_{\mathrm{q}}^{*}$ and $W_{\mathrm{q}}$ by eqs. (2.10), (2.11), we can now express the total average energy of the nucleus as a functional of the spatial densities $\rho_{\mathrm{q}}$ only. The idea then is, as discussed in the introduction, to perform a variational calculation on the densities $\rho_{\mathbf{q}}$, including Lagrange multipliers $\lambda_{\mathrm{q}}$ to ensure the correct particle numbers $(N$ and $Z)$ :

$$
\delta \int \mathrm{d}^{3} r\left\{\mathscr{E}\left[\rho_{\mathrm{n}}, \rho_{\mathrm{p}}\right]-\lambda_{\mathrm{n}} \rho_{\mathrm{n}}(r)-\lambda_{\mathrm{p}} \rho_{\mathrm{p}}(r)\right\}=0
$$


(Here $\mathscr{E}\left[\rho_{\mathrm{n}}, \rho_{\mathrm{p}}\right]$ contains both the nuclear and the Coulomb parts.) In the following we shall discuss what happens if the variation is done exactly, i.e. if the corresponding Euler-Lagrange equations are solved. To simplify the presentation, we shall again assume only one kind of particle - realistically, one will obtain two coupled differential equations for $\rho_{\mathrm{n}}$ and $\rho_{\mathrm{p}}$-and leave out the effective mass and spin-orbit contributions (i.e. put $f=1$ and $W=0$ ). These restrictions do not affect the conclusions drawn below.

The Euler-Lagrange equation then becomes

$$
\frac{\hbar^{2}}{2 m}\left\{\frac{5}{3} \kappa \rho^{2 / 3}+\frac{1}{36}\left[\frac{(\nabla \rho)^{2}}{\rho^{2}}-2 \frac{\Delta \rho}{\rho}\right]+D_{4}[\rho]\right\}+V[\rho]=\lambda,
$$

where the terms in curly brackets come from the variation of the kinetic energy and the potential is given by (cf. eq. (2.9)) $V[\rho]=\delta \varepsilon_{\mathrm{pot}} / \delta \rho$. The kinetic term $D_{4}[\rho]=\delta \tau_{4} / \delta \rho$ contains 7 contributions with up to four derivatives of $\rho$ and is given explicitly in appendix A. The equation (3.23) can in general only be solved numerically. However, it is possible to determine rather easily the asymptotic behaviour of the solution both inside the nucleus and in the outer surface. ${ }^{*}$

\subsubsection{Asymptotic behaviour in the outer surface}

The fall-off of the density $\rho(r)$ at large distance $r$ (we shall for simplicity assume spherical symmetry) is completely determined by the gradient corrections in the kinetic energy functional $\tau[\rho]$, if they are included at all. We shall accordingly discuss it in three steps.

1. Using $\tau_{\mathrm{TF}}[\rho]$ only: If only $\tau_{\mathrm{TF}}[\rho]$ is used, eq. (3.23) reduces to

$$
\frac{\hbar^{2}}{2 m} \frac{5}{3} \kappa \rho^{2 / 3}+V \rho \rho=\lambda
$$

If the potential $V[\rho]$ contains only powers of $\rho$ and no gradients, the only solution of eq. (3.25) is $\rho(r)=\rho_{0}$ and one obtains thus a liquid drop model type constant density with a sharp cut-off at the surface.

For Skyrme-like forces with a term $b(\nabla \rho)^{2}$ in the potential energy, eq. (3.25) leads to a density profile which near the surface goes like [2]

$$
\rho(r) \propto \operatorname{Tgh}^{2}\left(\frac{r-R_{0}}{a}\right)
$$

for spherical nuclei, where $a$ is essentially determined by the constant $b$ in front of the $(\nabla \rho)^{2}$ term. This density thus has to be cut-off at a finite radius $r=R_{0}$ and put equal to zero outside, and is therefore not very physical. It leads to the deficiencies of the calculations reported in ref. [13] which we have already mentioned in the introduction.

2. Using $\tau_{\mathrm{TF}}[\rho]+\tau_{2}[\rho]$ : Berg and Wilets [10] pointed out that the inclusion of a Weizsäcker term in the variational equation (i.e. eq. (3.23) with $D_{4}=0$ ) leads to an asymptotic fall-off of the density with the correct exponential form (in the spherical case):

\footnotetext{
* For an exact solution without fourth order terms see the end of section 6.5, and with fourth order terms see ref. [112].
} 


$$
\rho(r) \underset{r \rightarrow \infty}{\longrightarrow} \frac{1}{r^{2}} \mathrm{e}^{-r / a}
$$

The range $a$ is given by the Fermi energy $\lambda$ (which is always negative) and the coefficient of the Weizsäcker term:

$$
a=\sqrt{-\frac{1}{36} \frac{\hbar^{2}}{2 m} \frac{1}{\lambda}} .
$$

Unfortunately, this range is too small by a factor $\sim 2-3$ compared with realistic nuclear surfaces. Consequently, the variational densities fall off too quickly in the outer surface and lead to an overestimation of the kinetic energy (which is partially compensated by an overestimation of the potential energy). This was confirmed in numerical calculations by Bohigas et al. [58], who solved the Euler equations using the local functional $\tau_{\mathrm{TF}}[\rho]+\tau_{2}[\rho]$, eqs. (3.14), (3.15), for a Skyrme force with $m_{\infty}^{*} / m \simeq 0.95$ and without spin-orbit force. The semiclassical energies obtained in this way differed from the exact HF energies by $\sim 0.4-0.6 \mathrm{MeV}$ per nucleon, thus by far more than the order of magnitude of the shell corrections.

To overcome this defect - still in an attempt to solve the relatively easy second order differential equation - several authors used functionals of the type

$$
\tau[\rho]=\alpha \rho^{5 / 3}+\eta \frac{1}{36} \frac{(\nabla \rho)^{2}}{\rho}+\frac{1}{3} \Delta \rho,
$$

where $\alpha$ and $\eta$ were adjustable parameters [10,34, 59-62]. In particular in the so-called MTF functional [59], $\eta$ was chosen to be $\sim 4-5$, in order to obtain realistic tails of the densities, see eq. (3.28). This leads, however, to a drastic overestimation of the kinetic energy-in particular its surface contributions - which was compensated in ref. [59] by reducing the coefficient of the TF term (i.e. $\alpha<\kappa)$. In this way it was possible to fit the kinetic energies of spherical nuclei quite well (see also ref. [60]). However, the price to be paid for this is that $\alpha$ and $\beta$ depend on the nucleon number and on the force (in particular on $m^{*}$ ). The latter is obvious since the explicit effective mass and spin-orbit contributions in $\tau_{2}[\rho]$, shown in eq. (3.20), are ignored in eq. (3.29). Moreover, the MTF functional [59] completely fails to give reasonable deformation energies due to a drastic overestimation of the surface energy contributions (see section 4.3 below).

Treiner and Krivine [61] used recently another functional of the type of eq. (3.29) with the original coefficient of the TF term (i.e. $\alpha=\kappa$ ) and $\eta=2$, and added the correct second order spin-orbit terms (see eqs. (3.20), (3.21)). This functional still slightly overestimates the surface energy, leading to a too high fission barrier as compared to the one obtained with the full, unchanged functional $\tau_{\text {ETF }}[\rho]$ including the 4 th order contribution (see section 4.3). In fact, one can easily estimate from the results presented in ref. [56] that in order to mock up the contributions from $\tau_{4}[\rho]$ to the kinetic energy both for spherical and deformed nuclear shapes, one would have to multiply $\tau_{2}[\rho]$ with a factor of $\sim 1.3-1.5$ (still, however, not taking care of the effective mass and spin-orbit contributions). But in that case, as well as for the functional of ref. [61], the tails of the density distributions again are too steep.

One faces thus a basic dilemma when using adjustable functionals of the type of eq. (3.29): if one wants to obtain densities with good tails, one needs $\eta \simeq 4-5$; if one wants to obtain good energies, and in particular deformation energies, one needs $\eta \approx 1.3-1.5$ (a similar dilemma exists also in atomic 
physics in the so-called Thomas-Fermi Weizsäcker theory, see ref. [63]). We shall see in the next section that this dilemma can be satisfactorily resolved by using the full, unchanged functional $\tau_{\mathrm{ETF}}[\rho]$.

3. Using $\tau_{\mathrm{ETF}}[\rho]$ up to 4th order: The full fourth order equation (3.23) was discussed in ref. [56]. In this case the spherical solution of $\rho(r)$ falls off like

$$
\rho(r) \underset{r \rightarrow \infty}{\longrightarrow} \frac{c}{r^{6}}
$$

the coefficient $c$ is given by

$$
c=\left[-\frac{\hbar^{2}}{2 m}\left(3 \pi^{2}\right)^{-2 / 3} \frac{13}{45} \frac{1}{\lambda}\right]^{3 / 2} .
$$

This result at first looks rather discouraging, since eq. (3.30) is not the behaviour we would like to expect from a nice density. However, we do not know at which distance from the nuclear surface the behaviour $r^{-6}$ will be assumed. In order to investigate this, let us take $\lambda \simeq-7 \mathrm{MeV}$. We then find from eq. (3.31) that $c \simeq 0.03 \mathrm{fm}^{3}$. If eq. (3.30) were to be true at a distance of $r=10 \mathrm{fm}$ in ${ }^{208} \mathrm{~Pb}$, the density then would be $3 \times 10^{-8} \mathrm{fm}^{3}$ at that point which is 4 orders of magnitude smaller than what it would be for a Fermi function type density. This indicates that eq. (3.30) is a purely mathematical result which is reached so far outside the nuclear surface that it will have no physical meaning. This will be illustrated also in the case of semi-infinite nuclear matter discussed in section 5.2 below.

Unfortunately, the highly nonlinear, fourth order differential equation (3.23) seems inaccessible to numerical solutions. Even in the semi-infinite case, where it can be integrated once analytically, we did not succeed in solving numerically the resulting third order equation. However, the results obtained with a restricted variational space for the densities $\rho_{\mathrm{q}}(\boldsymbol{r})$ presented in section 4 below are satisfactory enough, so that it does not seem to be necessary to solve eq. (3.23) exactly.*

\subsubsection{Asymptotic behaviour inside the nucleus}

The onset of the surface region, i.e. the asymptotic deviation from a constant value of the density in a heavy nucleus, can also be estimated qualitatively without exactly solving the Euler equation. For simplicity we shall ignore the Coulomb interaction and the curvature effects, i.e. take the limit of a very large nucleus (or the surface profile of semi-infinite nuclear matter). Since in the inner region the density is very near its saturation value, we shall - following Skyrme [2] and Strutinsky and Tyapin [64] who developed in this way a precursor of the droplet model-replace the Skyrme energy density by a schematic one which, however, preserves the correct saturation properties.

We thus write

$$
\mathscr{E}[\rho]=\rho \tilde{e}_{\infty}(\rho)+b(\nabla \rho)^{2}+\frac{\hbar^{2}}{2 m}\left(\tau_{2}[\rho]+\tau_{4}[\rho]\right)
$$

where the "volume part" $\tilde{e}_{\infty}(\rho)$ instead of eq. (2.12) is taken to be

$$
\tilde{e}_{\infty}(\rho)=a_{\mathrm{v}}^{\infty}+\frac{K_{\infty}}{18 \rho_{\infty}^{2}}\left(\rho-\rho_{\infty}\right)^{2}
$$

\footnotetext{
* The full fourth order equation was recently solved numerically in ref. [112].
} 
This corresponds to a parabolic approximation of the saturation curve near the saturation density $\rho_{\infty}$, which certainly is good enough for the following estimations. Writing

$$
\rho(r)=\rho_{\infty} y(r)
$$

the Euler equation then becomes (neglecting the curvature contribution)

$$
\frac{K_{\infty}}{18}\left(3 y^{2}-4 y+1\right)-2 b \rho_{\infty} y^{\prime \prime}+\frac{\hbar^{2}}{2 m}\left(D_{2}[y]+D_{4}[y]\right)=0
$$

where $y^{\prime \prime}=\mathrm{d}^{2} y / \mathrm{d} r^{2}$ and $D_{2}[y]$ and $D_{4}[y]$ are again the variational derivatives of the gradient corrections to $\tau_{\text {ETF }}[\rho]$. The latter play a minor role in the following development and we shall therefore drop $D_{4}[\rho]$ immediately. To arrive at eq. (3.35) we have also neglected the fact that the central density $\rho_{0}$ is in general different from the saturation density $\rho_{\infty}$; this has little bearing on the following argument and shall be dealt with in section 5 .

We now write

$$
y(r)=1-\mathrm{e}^{(r-R) / \alpha}=1-\varepsilon(r) .
$$

Inside the nucleus, $\varepsilon(r) \ll 1$ and we can expand eq. (3.35) in powers of $\varepsilon$. Keeping the linear terms in $\varepsilon$, we obtain an equation for $\alpha$ :

$$
\alpha=\sqrt{\frac{18}{K_{\infty}}\left(b \rho_{\infty}+\frac{1}{36} \frac{\hbar^{2}}{2 m}\right)} .
$$

For realistic Skyrme forces, $b \rho_{\infty} \simeq 10-13 \mathrm{MeV} \mathrm{fm}^{2}$, so that $\alpha$ turns out to be of the order of $\sim 1 \mathrm{fm}$. The Weizsäcker correction (the second term in the brackets in eq. (3.37)) only contributes $\sim 3 \%$ to this result, the term $D_{4}[\rho]$ in eq. (3.35) would have contributed even far less.

We learn from this that the asymptotic inner part of the nuclear surface is mainly determined by the "surface term" $b(\nabla \rho)^{2}$ of the Skyrme energy density and by the incompressibility $K_{\infty}$; the kinetic energy plays only a minor role here. The range $\alpha$ of the inner surface part is $\sim 1 \mathrm{fm}$ and thus about twice larger than the typical value of the diffuseness parameter of the density when parametrized by a Fermi function. This tends to make the realistic densities asymmetric around the half-density distance $r_{1 / 2}$ (at which $\left.\rho\left(r_{1 / 2}\right)=\rho_{0} / 2\right)$; the "shoulder" of the surface is broader than the tail of the surface. This asymmetry is, indeed, seen in the averaged HF results discussed in section 4.2 below.

In order to summarize this section, let us repeat our main conclusions:

1. The semiclassical $\hbar$-expansion allows to determine the average energy of a system of fermions in a smooth (analytical) potential $V(r)$ in the form of a fast converging asymptotic series. All diverging terms - which usually show up at 6th and higher orders in $\hbar$-must be left out; they constitute the fluctuating (shell-correction) part of the energy. The semiclassical energy is for all practical purposes identical to the microscopically Strutinsky-averaged energy.

2. If realistic densities $\rho(r)$ with an exponential fall-off are used, the ETF kinetic energy density functional $\tau_{\mathrm{ETF}}[\rho]$ contains only gradient terms up to 4 th order, all higher order terms diverging again. In tests with Strutinsky-averaged densities, this functional reproduces to within less than $\sim 1.5 \mathrm{MeV}$ the average kinetic energy (for one kind of nucleons). 
3. In variational calculations, heuristic functionals $\tau[\rho]$ with only second order corrections and adjustable coefficients can either reproduce energies or density profiles, but not both at the same time.

4. If the functional $\tau_{\mathrm{ETF}}[\rho]$ is used to solve the Euler-Lagrange variational equation for the density, the gradient corrections to $\tau_{\text {ETF }}[\rho]$ completely determine the asymptotic fall-off of the density in the extreme surface. In no order of its gradient expansion can $\tau_{E T F}[\rho]$ give a realistic exponential fall-off. In particular with the gradient terms kept up to 4 th order, one obtains a fall-off of the form $1 / r^{6}$.

5. This latter result need not be in contradiction with the positive numerical results quoted in point 2 in the sense that the mathematical fall-off $\sim 1 / r^{6}$ is only assumed at far distances outside the nucleus which play no physical role, whereas the physically important surface region is compatible with the 4th order functional $\tau_{\mathrm{ETF}}[\rho]$.

6. Practically independent of the gradient corrections to $\tau[\rho]$, the inner asymptotic part of the surface is essentially determined by a balance between the gradient term $\sim(\nabla \rho)^{2}$ of the potential energy and the incompressibility $K_{\infty}$. As a consequence, the density profile is in general asymmetric around its inflection point.

\section{Semiclassical variational calculations for finite nuclei}

This section is devoted to a presentation of the results of variational calculations for various nuclear bulk properties using the density functional method outlined in section 3 . As we have seen there, the exact variation eq. (3.22) of the total Skyrme energy using the ETF functionals leads to two coupled, highly nonlinear fourth order differential equations which seem hopeless to solve exactly, even numerically. On the other hand, we have gained complete confidence in the ability of the functional $\tau_{\mathrm{ETF}}[\rho]$ to reproduce the correct average kinetic energies for densities which are close to the ones we would expect in microscopical (HF) calculations.

We therefore decided to perform the variation eq. (3.22) in a restricted variational space by parametrizing the densities $\rho_{\mathrm{q}}\left(r ; \lambda_{i}\right)$ and minimizing the total energy $E\left[\rho_{\mathrm{n}}, \rho_{\mathrm{p}}\right]$ with respect to the variational parameters $\lambda_{i}$. Preliminary calculations of this kind have already been performed earlier [65]; however, only the second order corrections to the functionals $\tau_{\text {ETF }}[\rho]$ and $J_{\text {ETF }}[\rho]$ were used and, correspondingly, the resulting density profiles were too steep in the outer surface, as we have seen in section 3.3 above.

In the following we shall first discuss the parametrisation of the densities and then present the numerical results.

\subsection{Parametrisation of the nuclear densities}

Our empirical knowledge of nuclear charge distributions, together with the conclusions drawn in section 3.3 above, encourage us to use modified Fermi functions as trial functions for the densities $\rho_{q}(r)$.

For spherical shapes we choose the radial form (see also ref. [65]):

$$
\rho_{\mathrm{q}}(r)=\frac{\overline{\rho_{0 \mathrm{q}}\left\{1+\rho_{1 \mathrm{q}}\right.} \overline{\left.\exp \left[-r^{2} / \beta_{\mathrm{q}}^{2} R_{\mathrm{q}}^{2}\right]\right\}}}{\left\{1+\exp \left[r-R_{\mathrm{q}} / \alpha_{\mathrm{q}}\right]\right\}^{\gamma_{\mathrm{q}}}} \quad(\mathrm{q}=\mathrm{n}, \mathrm{p})
$$

where the index $\mathrm{q}$ is again used to denote neutrons and protons. We have thus 10 variational parameters: $\rho_{0 \mathrm{q}}, \rho_{1 \mathrm{q}}, \beta_{\mathrm{q}}, \alpha_{\mathrm{q}}$ and $\gamma_{\mathrm{q}}(\mathrm{q}=\mathrm{n}, \mathrm{p})$; the radius constants $R_{\mathrm{q}}$ are always determined to fix the 
nucleon numbers $Z$ and $N$. For $\rho_{1 \mathrm{q}}=0$ and $\gamma_{\mathrm{q}}=1$, eq. (4.1) reduces to the familiar Fermi function with central density $\rho_{0 \mathrm{q}}$ and surface diffuseness $\alpha_{\mathrm{q}}$.

The generalized form eq. (4.1) opens two extra degrees of freedom: first, an asymmetry of the surface around $r=R_{\mathrm{q}}$ is generated for values of $\gamma_{\mathrm{q}}$ different from unity. In section 3.3 above, we have given reasons why we have to expect $\gamma_{\mathrm{q}}>1$; this asymmetry of the surface profile will also be further discussed in section 5.1 below, when considering the case of semi-infinite nuclear matter. Second, the numerator of eq. (4.1) allows for a depression $\left(\rho_{1 \mathrm{q}}<0\right)$ or an enhancement $\left(\rho_{1 \mathrm{q}}>0\right)$ at the centre with a range measured by the parameter $\beta_{\mathrm{q}}$. For this range to be physical, $\beta_{\mathrm{q}}$ should be limited to values

$$
\sim 0.3<\beta_{\mathrm{q}}<1
$$

For too small values of $\beta_{\mathrm{q}}$, a central bump or hole would resemble a shell effect, which however cannot be reproduced in this semiclassical framework; for $\beta_{\mathrm{q}}$ of the order of 1 or larger, the exponential factor would dominate the form of the density and destroy the surface region defined by the denominator of eq. (4.1). In ref. [65] it was found, indeed, that the total energy was practically independent of $\beta_{\mathrm{q}}$ in the range $\sim 0.4<\beta_{\mathrm{q}}<0.6$ (for those cases where $\rho_{\mathrm{q}_{1}} \neq 0$ was favoured at all), so that one could put $\beta_{\mathrm{q}}=0.5$ in all practical cases. This has also been verified in the present calculations.

In this semiclassical model, all stable nuclei are spherical in their ground state, as it is the case also in the pure liquid drop model. To obtain deformation energies (or the ground states of the deformed nuclei after inclusion of shell effects), one thus has to add an external constraint in the variational calculation. This could, in principle, be done with the help of Lagrange multipliers and various multipole fields. It appears, however, more appealing and at the same time easier to exploit the experience gained in liquid drop model (LDM) plus shell-correction calculations for fission barriers [37]. There a technique was adopted to define deformed Woods-Saxon potentials with a constant surface diffuseness $[66,67]$.

The idea is to start from a "generating LDM surface", given by a shape function $\pi\left(r, z ; \delta_{i}\right)$ :

$$
\pi\left(r, z ; \delta_{i}\right)=0
$$

Hereby $(r, z)$ are cylindrical coordinates (we shall assume axial symmetry around the $z$-axis throughout this paper) and $\delta_{i}$ are a suitable set of deformation parameters (e.g. the $(c, h)$ shape parameters used for fission calculations in refs. [37,67]). We then assume that the density varies across the LDM surface, given by eq. (4.3), in the same way as along the radial variable $r$ in the spherical case. This may be achieved [66] by replacing $r$ in eq. (4.1) by $R_{\mathrm{q}}+l$, defining

$$
r-R_{\mathrm{q}} \rightarrow l=\pi\left(r, z ; \delta_{i}\right) /\left|\nabla_{r, z} \pi\left(r, z ; \delta_{i}\right)\right|
$$

This yields automatically a constant gradient of the density orthogonal to the LDM surface (i.e. the half-density surface in case $\gamma_{\mathrm{q}}=1$ ). The implicit assumption of a surface diffuseness $\alpha_{\mathrm{q}}$ independent of the local curvature suggests itself from the well-known experimental fact that it is nearly constant for the measured charge distributions, throughout the mass table.

In earlier preliminary calculations $[68,69]$, we have adopted this technique to define deformed diffuse density distributions, using the $(c, h)$ family of fission shapes $[37,67]$. The semiclassical fission barriers obtained there were higher than expected from microscopically averaged HF calculations. The discrepancy could be traced back to the fact that the spherical density profiles obtained with eqs. (4.3), (4.4) and with the $(c, h)$ shapes are not Fermi functions (for $\rho_{1 \mathrm{q}}=0, \gamma_{\mathrm{q}}=1$ ), but 


$$
\rho_{\mathrm{q}}(r)=\rho_{0 \mathrm{q}}\left[1+\exp \left(l / \alpha_{\mathrm{q}}\right)\right]^{-1} \underset{\text { spher. }}{\longrightarrow} \rho_{0 \mathrm{q}}\left[1+\exp \left(\frac{r^{2}-R_{q}^{2}}{2 r \alpha_{\mathrm{q}}}\right)\right]^{-1},
$$

which minimize the total energy much less. We later found that the "hard $\pi$ " modification [37] of the prescription eq. (4.4), which leads to Fermi functions in the spherical case, also leads to lower fission barriers in the variational calculations.

In the present work we have adopted still another definition which is both more appealing and brings essential technical advantages in the numerical integration for the deformed shapes. Instead of the variable $l$ (eq. (4.4)) we use the coordinate $u$ of a curvilinear coordinate system $(u, v, \varphi)$ which follows the deformation of the LDM surface and was originally proposed by Strutinsky and Tyapin [64]. Hereby $u$ is the distance normal to the sharp LDM surface from a given point in space. The deformed densities are then defined replacing $r$ in eq. (4.1) by $R_{\mathrm{q}}+u$. The details of this "intrinsic" coordinate system will be discussed in appendix $\mathrm{C}$.

\subsection{Bulk properties of spherical nuclei}

We now present the variational semiclassical results for binding energies, densities and radii of spherical nuclei and compare them both to HF results and to experiment. We used mostly the Skyrme force $\mathrm{SkM}^{*}$ which will be discussed in detail in section 4.4 .

The full variation of the 10 independent parameters in eq. (4.1) was done for a series of nuclei presented in table 3 . There, all the parameters are listed together with the minimized total energy $E_{\text {ETF }}$. For ${ }^{40} \mathrm{Ca}$ and ${ }^{208} \mathrm{~Pb}$ we also give the results obtained when the densities were restricted to pure Fermi functions (imposing $\gamma_{p}=1, \rho_{1 q}=0$ ) or asymmetric Fermi functions (with $\gamma_{q} \neq 1$, but $\rho_{1 q}=0$ ). It is interesting to note that the 10 -parameter variation lowers the total energy by only $2.2 \mathrm{MeV}$ in ${ }^{40} \mathrm{Ca}$ (i.e. $\sim 0.7 \%$ ) and by $4.7 \mathrm{MeV}$ in ${ }^{208} \mathrm{~Pb}$ (i.e. $\sim 0.3 \%$ ) compared to the 4-parameter variation with pure Fermi functions. Furthermore, almost all of this gain in energy is already obtained with flat densities $\left(\rho_{1 q}=0\right)$ with an asymmetric surface $\left(\gamma_{\mathrm{q}} \neq 1\right)$.

As long as one is interested in binding or deformation energies alone, it is thus perfectly sufficient to use 3-parameter densities (i.e. Fermi functions to the power $\gamma_{\mathbf{q}}$ ) with a flat interior part; the building-up of a dip or a bump in the interior lowers the energy by less than $0.5 \mathrm{MeV}$ in the whole mass region. The surface asymmetry parameters $\gamma_{\mathbf{q}}$ found for the force $\mathrm{SkM}^{*}$ vary only little, slightly increasing from $\sim 1.4$ in light to $\sim 1.5-1.6$ in heavy nuclei (in the limit $A \rightarrow \infty$, i.e. for semi-infinite matter, $\gamma$ reaches the value 1.78 , see section 5.1); the values $\gamma_{\mathrm{n}}$ are only slightly larger than $\gamma_{\mathrm{p}}$. For forces with larger incompressibilities $K_{\infty}, \gamma_{q}$ becomes smaller, as can easily be understood on the basis of the discussion in section 3.3 above (cf. the force SIII where $\gamma_{\mathrm{q}} \sim 1.2$ ). We are aware that the quantities $\gamma_{\mathrm{q}}$ are difficult to reach experimentally because of the superposition of the shell effects in the real nuclei. However, when comparing different theoretical approaches, these quantities are useful. In refs. $[61,65]$ much larger values, $\gamma_{\mathrm{q}} \sim 2-3$, were obtained. This is due to the inclusion of second order corrections only in $\tau[\rho]$ in those variational calculations which, as we have seen in section 3.3 , leads to a too steep fall-off of the densities in the tail region and thus tends to exaggerate the asymmetry of the surface profile. Therefore, the values of $\gamma_{\mathbf{q}}$ are actually a rather crucial measure for the quality of the density profiles.

That the densities obtained with the present approach do have realistic surfaces is demonstrated in fig. 1, where we compare the profiles obtained for ${ }^{40} \mathrm{Ca}$ and ${ }^{208} \mathrm{~Pb}$ with the microscopical HF results, both calculated with the same force $S k M^{*}$. An almost perfect agreement is obtained in the surface and the tail region. In the interior part the ETF densities reproduce nicely the average trend of the HF 
Table 3

Variational parameters obtained with the $\mathbf{S k M}^{*}$ force for the densities of various spherical nuclei. Parametrisation: eq. (4.1). $\rho_{0 q}$ and $\rho_{1 q}$ are in $\mathrm{fm}^{-3}, \alpha_{\mathrm{q}}$ and $R_{\mathrm{q}}$ in $\mathrm{fm}$. Values marked by an asterisk (*) were imposed in the variation. $E_{\mathrm{ETF}}$ is the total variational energy in $\mathrm{MeV}$

\begin{tabular}{lllllll}
\hline & $\rho_{0 \mathrm{p}}$ & $\rho_{0 \mathrm{n}}$ & $\alpha_{\mathrm{p}}$ & $\alpha_{\mathrm{n}}$ & $\gamma_{\mathrm{p}}$ & $\gamma_{\mathrm{n}}$ \\
\hline${ }^{40} \mathrm{Ca}$ & 0.0776 & 0.0804 & 0.472 & 0.464 & $1.0^{*}$ & $1.0^{*}$ \\
& 0.0804 & 0.0834 & 0.577 & 0.580 & 1.43 & 1.49 \\
& 0.0768 & 0.0777 & 0.566 & 0.553 & 1.42 & 1.42 \\
${ }^{208} \mathrm{~Pb}$ & 0.0611 & 0.0888 & 0.442 & 0.528 & $1.0^{*}$ & $1.0^{*}$ \\
& 0.0623 & 0.0912 & 0.535 & 0.658 & 1.42 & 1.56 \\
& 0.0639 & 0.0904 & 0.557 & 0.646 & 1.45 & 1.50 \\
${ }^{58} \mathrm{Ni}$ & 0.0756 & 0.0800 & 0.561 & 0.557 & 1.42 & 1.45 \\
${ }^{116} \mathrm{Sn}$ & 0.0697 & 0.0870 & 0.559 & 0.605 & 1.48 & 1.50 \\
${ }^{124} \mathrm{Sn}$ & 0.0664 & 0.0902 & 0.551 & 0.630 & 1.48 & 1.50 \\
\hline & $\rho_{1 \mathrm{p}}$ & $\rho_{1 \mathrm{n}}$ & $\beta_{\mathrm{n}}=\beta_{\mathrm{p}}$ & $R_{\mathrm{p}}$ & $R_{\mathrm{n}}$ & $E_{\mathrm{ETF}}$ \\
\hline${ }^{40} \mathrm{Ca}$ & $0.0^{*}$ & $0.0^{*}$ & - & 3.761 & 3.720 & -327.60 \\
& $0.0^{*}$ & $0.0^{*}$ & - & 3.999 & 3.984 & -329.42 \\
& 0.136 & 0.199 & 0.5 & 4.027 & 4.001 & -329.78 \\
${ }^{208} \mathrm{~Pb}$ & $0.0^{*}$ & $0.0^{*}$ & - & 6.748 & 6.839 & -1603.8 \\
& $0.0^{*}$ & $0.0^{*}$ & - & 6.975 & 7.194 & -1608.3 \\
& -0.086 & -0.004 & 0.5 & 6.976 & 7.125 & -1608.5 \\
${ }^{58} \mathrm{Ni}$ & 0.107 & 0.168 & 0.5 & 4.550 & 4.552 & -491.6 \\
${ }^{116} \mathrm{Sn}$ & 0.019 & 0.078 & 0.5 & 5.734 & 5.854 & -976.3 \\
${ }^{124} \mathrm{Sn}$ & 0.017 & 0.061 & 0.5 & 5.828 & 5.990 & -1036.8 \\
\hline & & & & & & \\
\hline
\end{tabular}

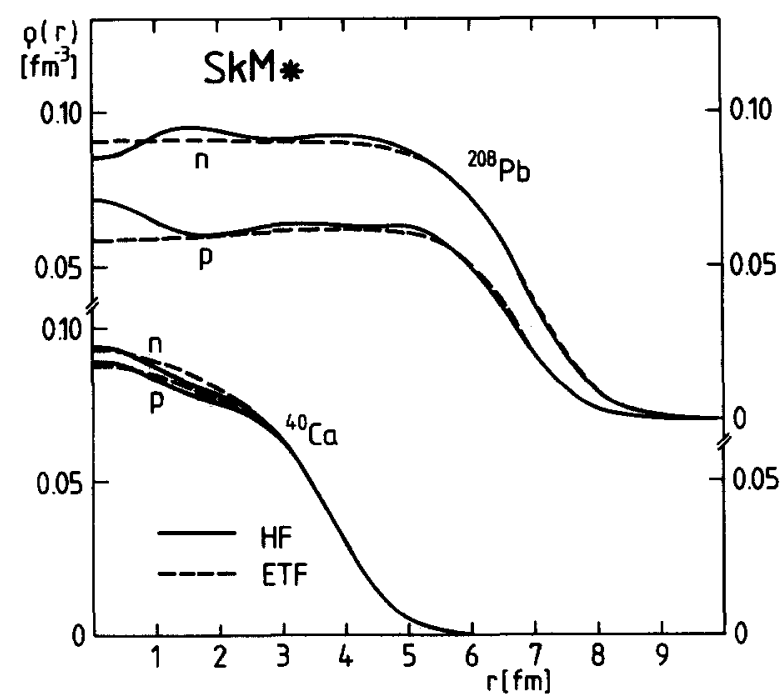

Fig. 1. Neutron and proton density profiles (in $\mathrm{fm}^{-3}$ ) calculated with the HF (solid lines) and the variational ETF method (dashed lines) using the $\mathrm{SkM}^{*}$ force. The parametrisation eq. (4.1) of the densities was used in the ETF calculations. 
results. In fact the possibility to build up a bump or a dip-although it does not affect the binding energies by more than a few hundred $\mathrm{keV}$ - is important for obtaining this agreement. In particular for ${ }^{40} \mathrm{Ca}$, where the central densities are enhanced by $\sim 20 \%$ there is no saturated bulk region such as in heavier nuclei. It is worth underlining that this central enhancement is not just a shell effect, but it is well reflected also in the semiclassical densities. It comes from the relative smallness of this nucleus where the surface region is dominating over the volume part. In heavy nuclei such as ${ }^{208} \mathrm{~Pb}$, the ratio of surface to volume region is small and a relatively constant, saturated central density builds up. The ${ }^{208} \mathrm{~Pb}$ proton density is slightly depressed at the centre by $\sim 8 \%$ due to the Coulomb repulsion. This dip, which is almost invisible for the neutron density ( $\$ 1 \%)$, is in both cases buried in the shell fluctuations of the quantum mechanical densities.

In fig. 2 we compare the semiclassical charge distributions-obtained by convoluting the proton densities with a proton form factor of $\left\langle r^{2}\right\rangle_{p}=0.64 \mathrm{fm}^{2}$ - of five spherical nuclei to the empirical distributions deduced from a recent analysis of the elastic electron scattering experiments at Saclay [70]. A surprisingly good agreement is found for the average trends in all cases. That the experimental charge distributions show much less fluctuations than the microscopical HF type distributions, is a well-known puzzle (see, e.g., ref. [71]), although a recent careful analysis [72] shows that by playing on the combination of gradient terms $\sim\left[\nabla\left(\rho_{\mathrm{n}}-\rho_{\mathrm{p}}\right)\right]^{2}$ in the Skyrme energy density - which can be handled by a suitable combination of the exchange parameters $x_{1}$ and $x_{2}$-one is able to reduce the size of the shell oscillations of the HF densities to that of the experimental ones. Apart from this question we can

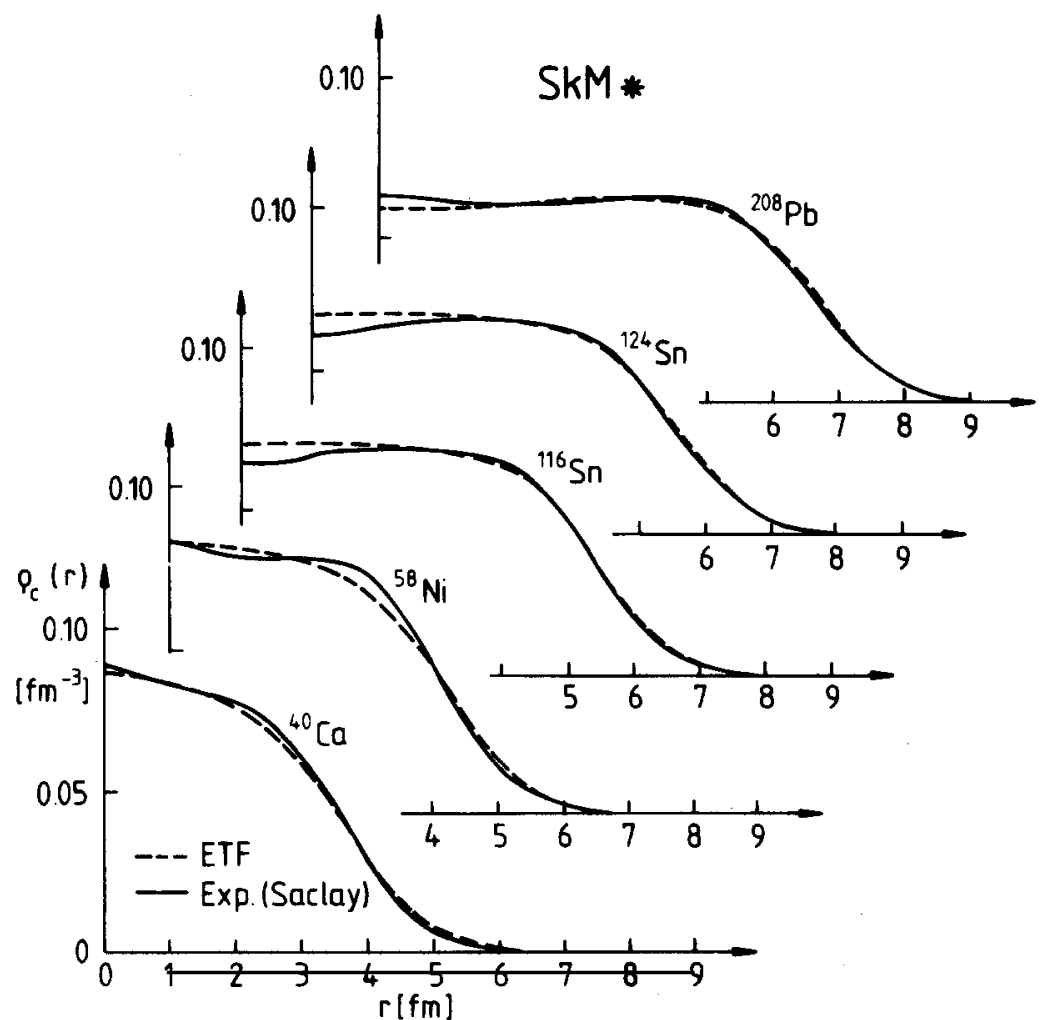

Fig. 2. Charge density distributions of spherical nuclei. Solid lines: experimental results [70]; dashed lines: results of variational ETF calculations with the SkM* force. 
conclude that our semiclassical approach is well suited for predicting average density profiles. The good agreement in fig. 2 should also be taken as a success of the SkM* parametrisation which for the density profiles is clearly superior to most older Skyrme forces.

In table 4 we list the binding energies obtained for 9 spherical nuclei with the SkM* force. We also show the experimental values and the HF results (taken from ref. [7]). An excellent agreement within $\sim 1 \mathrm{MeV}$ is observed for all stable nuclei. The overbinding of several $\mathrm{MeV}$ in neutron-rich isotopes and a lack of binding of a few $\mathrm{MeV}$ in the neutron-poor isotopes away from the $\beta$-stability line indicates that the $\mathrm{SkM}^{*}$ force does not have optimal asymmetry properties (see also the discussion in section 4.4 below and in ref. [7]). The ETF binding energies of course contain no shell effects and should only be compared to the averaged HF energies. In the quantities $B^{\mathrm{EVM}}$, shown in the last column of table 4 , the shell effects were included perturbatively, as will be discussed in section 4.5 below. The energies $B^{\text {ETF }}$ are too large compared to the averaged HF energies by $\sim 2-4 \mathrm{MeV}$ in lighter and by $\sim 4-8 \mathrm{MeV}$ in heavier nuclei. This effect of a slight overbinding was observed earlier with other Skyrme forces [69]-it is larger by a factor of roughly 2 for the SIII force, presumably due to its larger incompressibility - and must be considered as a slight defect of the ETF functionals. Although the variational principle holds strictly, according to Hohenberg and Kohn [14], for the "ideal" (but unknown) exact functional $\mathscr{E}[\rho]$, the use of approximate functionals may lead to violations of the variational principle and thus to such overbinding effects. This slight deficiency of $B^{\mathrm{ETF}}$ is, however, healed after inclusion of the shell effects by the "expectation value method" (EVM) discussed in section 4.5 .

Table 4

Binding energies (in $\mathrm{MeV}$ ) of spherical nuclei, obtained with the $\mathrm{SkM}^{*}$ force

\begin{tabular}{lrrrr}
\hline & \multicolumn{1}{c}{$B^{\text {exp }}$} & \multicolumn{1}{c}{$B^{\text {HF }}$} & \multicolumn{1}{c}{$B^{\text {ETF }}$} & $B^{\text {EVM }}$ \\
\hline${ }^{16} \mathrm{O}$ & 127.6 & 127.7 & 128.0 & 127.4 \\
${ }^{40} \mathrm{Ca}$ & 342.1 & 341.1 & 345.9 & 340.4 \\
${ }^{48} \mathrm{Ca}$ & 416.0 & 420.1 & 421.8 & 418.4 \\
${ }^{56} \mathrm{Ni}$ & 484.0 & 485.4 & 483.9 & 483.1 \\
${ }^{90} \mathrm{Zr}$ & 783.9 & 784.5 & 786.6 & 782.7 \\
${ }^{114} \mathrm{Sn}$ & 971.6 & 969.2 & 976.0 & 967.9 \\
${ }^{132} \mathrm{Sn}$ & 1102.7 & 1110.7 & 1101.5 & 1108.3 \\
${ }^{140} \mathrm{Ce}$ & 1172.7 & 1173.9 & 1174.5 & 1171.6 \\
${ }^{208} \mathrm{~Pb}$ & 1636.5 & 1636.4 & 1627.0 & 1633.7 \\
\hline
\end{tabular}

The fact that the binding energies $B^{\mathrm{ETF}}$ in table 4 are larger than the corresponding energies $\left(-E_{\mathrm{ETF}}\right)$ in table 3 is due to the inclusion here of a centre-of-mass energy correction:

$$
\Delta E_{\text {c.m. }}=-\frac{\left\langle p^{2}\right\rangle}{2 m A}=-\frac{E_{\text {kin }}}{A}
$$

as it has been usual in HF calculations [25,30]. Since it is included in this form in the HF results [7] reported in tables 4 and 5 , we also included $\Delta E_{\text {c.m. }}$ (eq. (4.6)) in the semiclassical calculations to these tables. A comment about this c.m. energy correction might be at order here. It is well known (see, e.g. refs. [73, 74]) that the full correction, containing a one-body term (eq. (4.6)) and a two-body exchange term, is much smaller than the one-body estimate (eq. (4.6)) alone (by $\sim 30-40 \%$ in light and by a factor of $\sim 2-3$ in heavy nuclei). The error made in heavy nuclei leaving out $\Delta E_{\text {c.m. }}$ completely is thus smaller 
Table 5

Charge radii $r_{\mathrm{c}}$ and skin thickness parameters $t$ (both in $\mathrm{fm}$ ) obtained for spherical nuclei with the SkM* force (see text for the quantity $X_{t}$ ). Density profiles in ETF calculation restricted to pure Fermi functions. (Units of parameters as in table 3.)

\begin{tabular}{lllllll}
\hline & $r_{\mathrm{c}}^{\text {exp }}$ & $r_{c}^{\mathrm{HF}}$ & \multicolumn{1}{l}{$\boldsymbol{r}_{\mathrm{c}}^{\mathrm{ETF}}$} & \multicolumn{1}{l}{$\boldsymbol{t}_{\mathrm{HF}}$} & \multicolumn{1}{c}{$t_{\mathrm{ETF}}$} & \multicolumn{1}{l}{$\boldsymbol{X}_{t}$} \\
\hline${ }^{16} \mathrm{O}$ & 2.73 & 2.81 & 2.78 & -0.03 & -0.02 & 0.45 \\
${ }^{40} \mathrm{Ca}$ & 3.49 & 3.52 & 3.47 & -0.05 & -0.04 & 0.36 \\
${ }^{48} \mathrm{Ca}$ & 3.48 & 3.54 & 3.54 & 0.16 & 0.17 & 0.64 \\
${ }^{56} \mathrm{Ni}$ & 3.75 & 3.77 & 3.81 & -0.06 & -0.05 & 0.31 \\
${ }^{90} \mathrm{Zr}$ & 4.27 & 4.30 & 4.29 & 0.05 & 0.07 & 0.75 \\
${ }^{114} \mathrm{Sn}$ & & 4.60 & 4.61 & 0.08 & 0.08 & 0.77 \\
${ }^{132} \mathrm{Sn}$ & & 4.73 & 4.75 & 0.23 & 0.24 & 0.58 \\
${ }^{140} \mathrm{Ce}$ & $4.88^{*}$ & 4.89 & 4.89 & 0.13 & 0.14 & 0.66 \\
${ }^{208} \mathrm{~Pb}$ & 5.50 & 5.51 & 5.53 & 0.17 & 0.17 & 0.62 \\
\hline & $\rho_{0 \mathrm{p}}$ & $\rho_{0 \mathrm{n}}$ & $\alpha_{\mathrm{p}}$ & $\alpha_{\mathrm{n}}$ & $R_{\mathrm{p}}$ & $R_{\mathrm{n}}$ \\
\hline${ }^{16} \mathrm{O}$ & 0.0789 & 0.0807 & 0.452 & 0.448 & 2.661 & 2.642 \\
${ }^{40} \mathrm{Ca}$ & 0.0789 & 0.0817 & 0.468 & 0.460 & 3.743 & 3.701 \\
${ }^{48} \mathrm{Ca}$ & 0.0706 & 0.0899 & 0.441 & 0.500 & 3.918 & 4.010 \\
${ }^{56} \mathrm{Ni}$ & 0.0779 & 0.0811 & 0.472 & 0.462 & 4.245 & 4.190 \\
${ }^{90} \mathrm{Zr}$ & 0.0710 & 0.0864 & 0.454 & 0.490 & 4.991 & 5.017 \\
${ }^{114} \mathrm{Sn}$ & 0.0692 & 0.0864 & 0.453 & 0.496 & 5.445 & 5.470 \\
${ }^{132} \mathrm{Sn}$ & 0.0621 & 0.0923 & 0.438 & 0.540 & 5.661 & 5.804 \\
${ }^{140} \mathrm{Ce}$ & 0.0657 & 0.0884 & 0.446 & 0.512 & 5.842 & 5.907 \\
${ }^{208} \mathrm{~Pb}$ & 0.0613 & 0.0891 & 0.441 & 0.529 & 6.743 & 6.831 \\
\hline
\end{tabular}

* Natural Ce $\left(88.5 \%{ }^{140} \mathrm{Ce}\right)$.

than when the approximation eq. (4.6) is used. That the latter was included in the present calculations was a matter of pure convention. For the fit of the force $\mathrm{SkM}^{*}$, this has no significance since it was shown [30] that the correction eq. (4.6) could easily be absorbed into the parameters of the Skyrme force. In the present semiclassical context, the full correction including the 2-body exchange contributions is unfortunately not accessible to evaluation within the ETF model, as is discussed in appendix B. This holds also for the spurious rotational energy corrections necessary in HF calculations for deformed nuclei. We have therefore consistently left out any such corrections in the semiclassical calculations presented in this paper, except in tables 4 and 5 for the sake of comparison with the HF results.

In table 5 we list the density parameters and r.m.s. radii of the same 9 spherical nuclei as in table 4. For an easier discussion of the average trends of these results, we have restricted here the variational space to that of simple Fermi functions (thus imposing $\gamma_{q}=1$ and $\beta_{q}=0$ ). The values of the r.m.s. radii change by less than $0.01 \mathrm{fm}$ in all cases when the full 5-parameter variational densities (eq. (4.1)) are used. As already noticed in ref. [7], the semiclassical charge radii $r_{c}^{\text {ETF }}$ reproduce the HF ones $r_{c}^{\text {HF }}$ within less than $2 \%$ in light and well below $1 \%$ in heavy nuclei. The HF values in turn are in the same agreement with the experimental charge radii $r_{c}^{\text {exp }}$. In fact, it was shown in ref. [7] that if centre-of-mass, electromagnetic spin-orbit and neutron charge distribution corrections are added to $r_{c}^{\mathrm{HF}}$, the agreement with $r_{c}^{\text {exp }}$ is in all cases shown in table 5 within $0.01 \mathrm{fm}$, even in the notorious case of ${ }^{48} \mathrm{Ca}$.

A quantity of special interest is the so-called "neutron skin thickness" 


$$
t=r_{\mathrm{n}}-r_{\mathrm{p}}=\left\langle r^{2}\right\rangle_{\mathrm{n}}^{1 / 2}-\left\langle r^{2}\right\rangle_{\mathrm{p}}^{1 / 2}
$$

which is, however, an experimentally difficult observable. Table 5 shows that the ETF variational model reproduces excellently the HF values of $t$. The quantity $t$ is slightly negative in light symmetric nuclei as a result of the Coulomb force which tends to spread the protons a little, and becomes increasingly positive with increasing neutron excess, reaching the theoretical value of $\sim 0.17 \mathrm{fm}$ in ${ }^{208} \mathrm{~Pb}$ and $\sim 0.25 \mathrm{fm}$ in the particularly asymmetric isotope ${ }^{132} \mathrm{Sn}$. A nice advantage of the semiclassical treatment is that we can easily see how this "neutron skin" is made up by a "bulk part", coming from the difference in the half-density radii $\boldsymbol{R}_{\mathbf{q}}$, and a "diffuseness part", coming from the difference in the diffuseness parameters $\alpha_{\mathrm{q}}$. In the leptodermous expansion (see eq. (C.25)) one obtains for Fermi distributions

$$
r_{\mathrm{q}}^{2}=\left\langle r^{2}\right\rangle_{\mathrm{q}} \approx \frac{3}{5} R_{\mathrm{q}}^{2}\left[1+\frac{7}{3} \pi^{2}\left(\alpha_{\mathrm{q}} / R_{\mathrm{q}}\right)^{2}\right]
$$

up to terms of order $\left(\alpha_{\mathrm{q}} / R_{\mathrm{q}}\right)^{4}$. Rewriting $t$ (eq. (4.7)) we obtain

$$
\begin{aligned}
t & =\frac{r_{\mathrm{n}}^{2}-r_{\mathrm{p}}^{2}}{r_{\mathrm{n}}+r_{\mathrm{p}}}=t_{\mathrm{b}}+t_{\mathrm{d}} \\
& \approx \frac{1}{\left(r_{\mathrm{n}}+r_{\mathrm{p}}\right)}\left[\frac{3}{5}\left(R_{\mathrm{n}}^{2}-R_{\mathrm{p}}^{2}\right)+\frac{7 \pi^{2}}{5}\left(\alpha_{\mathrm{n}}^{2}-\alpha_{\mathrm{p}}^{2}\right)\right] .
\end{aligned}
$$

Equation (4.9) reproduces the exact values of $t_{\text {ETF }}$ within a few percent even for ${ }^{16} \mathrm{O}$. In table 5 we show the values of the ratio $X_{t}$ defined as

$$
X_{t}=t_{\mathrm{d}} / t
$$

which gives the percentage of the diffuseness contribution. We see that it is roughly $\sim 30-45 \%$ in symmetric nuclei and $\sim 60-75 \%$ in asymmetric nuclei. Thus, the diffuseness of the nuclear surface plays an important role - as one should expect - in determining the neutron skin.

We emphasize this point because in the droplet model [19], in which the quantity $t$ plays an important role, the degrees of freedom $\alpha_{\mathrm{q}}$ are neglected, and one cannot therefore expect this model to make quantitative predictions of the neutron skin (see section 5.3 for a more detailed discussion).

As the experimental situation is concerned, there is very poor information on $t$, since the neutron distributions are very little known and model dependent. There are three recent analyses of elastic proton scattering [75-77] and an older one of $\alpha$-scattering data [78] which, unfortunately, come to partially contradictory conclusions. We refer to ref. [76] for a detailed discussion of this delicate problem. If we take rough averages of all the quoted results, we find from these analyses:

$$
\begin{aligned}
& t_{\text {exp }} \simeq-0.02 \pm 0.05 \mathrm{fm} \text { for }{ }^{40} \mathrm{Ca} \\
& t_{\text {exp }} \simeq 0.2 \pm 0.2 \mathrm{fm} \text { for }{ }^{48} \mathrm{Ca} \\
& t_{\text {exp }} \simeq 0.1 \pm 0.1 \mathrm{fm} \text { for }{ }^{90} \mathrm{Zr} \\
& t_{\text {exp }} \simeq 0.1 \pm 0.2 \mathrm{fm} \text { for }{ }^{208} \mathrm{~Pb}
\end{aligned}
$$

Even though the error bars are large, we can say that the experimental values $t_{\exp }$ on the average seem to follow the trends of the theoretically predicted values. 


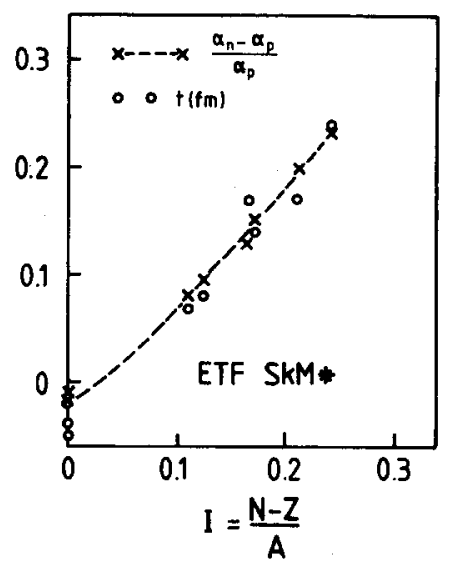

Fig. 3. Circles: neutron skin thickness $t$ (in $\mathrm{fm}$ ) of a series of spherical nuclei, plotted versus asymmetry $I$. Crosses: relative difference of surface diffuseness parameters of the same nuclei. All results obtained in ETF calculations with the SkM* force.

The well-known approximate constancy of the experimental charge surface diffuseness throughout the mass table is well reproduced in our semiclassical results: the values of $\alpha_{\mathrm{p}}$ are constant within less than $8 \%$. (Note that it is very difficult to verify this with HF results because of the shell effects!). The neutron values $\alpha_{n}$, however, are strongly correlated with the relative neutron excess $I=(N-Z) / A$. In fact, when plotting $\left(\alpha_{n}-\alpha_{p}\right) / \alpha_{p}$ versus $I$ one finds an almost perfect linear correlation, as shown in fig. 3 by the crosses. It is interesting that the neutron skin parameter $t$, shown in fig. 3 by circles (on the same scale, but in units of $\mathrm{fm}$ ), also approximately follows this correlation. This demonstrates once more the close connection between the surface diffuseness and the "neutron skin".

\subsection{Deformation energies and fission barriers of heavy nuclei}

We have already mentioned in the introduction that the fission barriers of actinide nuclei provided a longstanding puzzle to the constrained HF method using effective interactions. The first calculation, done some 10 years ago with the Skyrme SIII force [4], reproduced qualitatively the correct features of the double-humped barrier of ${ }^{240} \mathrm{Pu}$, including the correct deformations (i.e. quadrupole moments) of both the ground and the isomeric state. However, the heights of the barriers were too large, even after careful estimations of corrections due to truncation effects, spurious rotational energies and the effects of nonaxial and left/right asymmetric deformations (see also ref. [5] for a detailed discussion). More recently, full HF-Bogolyubov calculations have been achieved $[79,80]$ using the effective interaction of Gogny [81] for the fission barrier of ${ }^{240} \mathrm{Pu}$. Again, the outer barrier was higher than the experimental one by several $\mathrm{MeV}$, although octopole deformations were included and spurious zero point energies subtracted [80].

This apparent puzzle-both the SIII and the Gogny forces give very satisfactory ground-state properties, also for deformed nuclei - could not easily be solved; a refit of the forces taking into account the fission barriers in HF calculations was practically excluded due to their enormous computer times. On the other hand, the experience from the liquid drop model (LDM) [82] tells us that the ground-state properties of the known nuclei alone do not give sufficiently accurate information on the surface properties to allow for reliable extrapolations to the large deformations encountered at the fission barriers of actinides; in fact, fission barriers are included in the fits to obtain the LDM parameters. 
There was thus a definite hope that the effective forces could be improved to yield correct fission barriers without spoiling the quality of the ground-state properties.

The semiclassical density-variation method provides a powerful and very economic tool to obtain average deformation energies. The computation times for deformed nuclei are more than two orders of magnitude shorter than with constrained HF calculations. This allows for systematic searches of effective forces, including calculations of the barriers.

The semiclassical fission barriers of course have no shell structure and therefore show only one smooth maximum as in the LDM. The empirical height of the LDM barrier of ${ }^{240} \mathrm{Pu}$ is known from shell-correction calculations to be $\sim 3.8 \mathrm{MeV}$ [37] and should be ideally reproduced by a semiclassical calculation with an effective force, since the magnitude of the shell effects is believed to depend rather little on the bulk properties of the force (see, e.g., ref. [5]).

The first semiclassical calculations of fission barriers of ${ }^{240} \mathrm{Pu}$, using the ETF functionals $\tau_{\text {ETF }}[\rho]$ and $J_{\mathrm{ETF}}[\rho]$, were presented in refs. [68, 69]. It was shown in ref. [69] that the set of Skyrme forces SIII-SVI [30] all lead to much higher barriers than the LDM value, in accordance with the HF result for SIII. The barrier of the Ska force of Köhler [33] was somewhat lower, and that of the SkM force [34] even realistic. (Note that all barriers in ref. [69] were too high due to an insufficient parametrisation of the densities, as discussed in section 4.1.)

With the improved density parametrisation using the "intrinsic" coordinates $(u, v)$ (see section 4.1 and appendix C), we have recalculated the barriers of ${ }^{240} \mathrm{Pu}$ with the forces SIII, Ska, SkM and SkM* discussed below. The results are shown in fig. 4. The $(c, h)$ shapes of ref. [37] were used; at each point $c$ the energy is minimized with respect to $h$. The same trend as in ref. [69] is observed for the barrier heights. There is a link between the incompressibilities of these forces (see table 1) and the barrier heights. This can qualitatively be understood: the stiffer the equation of state, the stiffer the surface and therefore the higher the surface energy and with it the barrier. In fact, our discussions in section 3.3 show that the surface diffuseness is inversely proportional to $\sqrt{K_{\infty}}$ (see also the schematic models in refs. $[89,92])$. This is, of course, only a rather crude argument; the surface energy depends also on other ingredients than $K_{\infty}$. This is demonstrated by the curve for the force $\mathrm{SkM}^{*}$ which was adjusted (see section 4.4) to the empirical LDM barrier height (shown by a cross in fig. 4); it has the same value for $K_{\infty}$ as SkM.

In fig. 5 we demonstrate the influence of the 4 th order gradient contributions of the ETF functionals

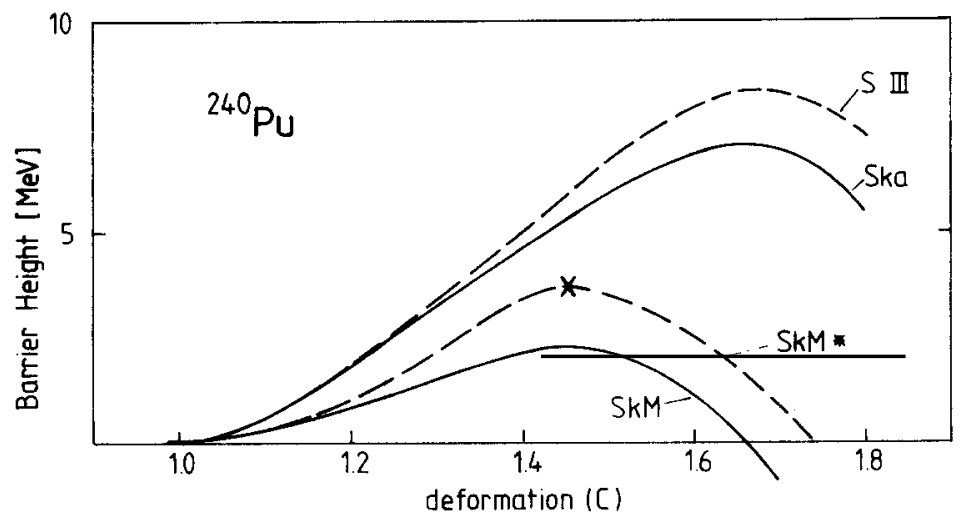

Fig. 4. Fission barriers obtained for ${ }^{200} \mathrm{Pu}$ with the ETF method using various Skyrme forces. $c$ is the elongation parameter [37]. The density profiles were asymmetric Fermi functions. [Eq. (4.1) with $\rho_{1 q}=0$.] The cross indicates the position of the empirical LDM saddle point [37]. 


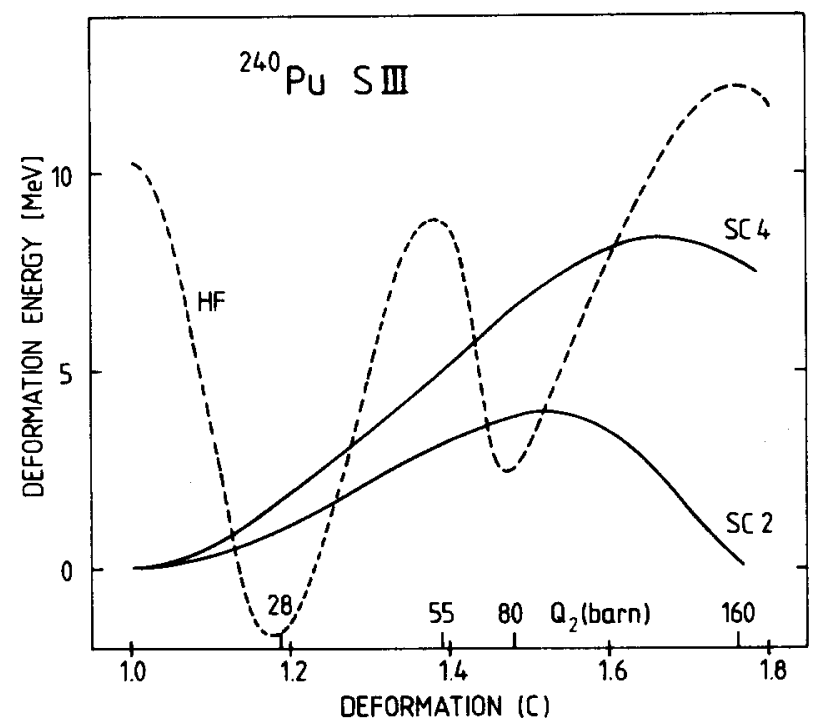

Fig. 5. As fig. 4 for the SIII force (solid lines). SC4: using complete ETF functionals up to 4th order. SC2: omitting 4th order gradient corrections. The dashed line is the result of a constrained HF calculation [17]. The mass quadrupole moments $Q_{2}$ are also given along the abscissa.

(i.e. $\tau_{4}[\rho]$ and $J_{4}[\rho]$ ) on the barrier height. The curve labeled SC4 is the full variational result obtained with all 4th order terms included; in the curve SC2, the contributions $\tau_{4}$ and $J_{4}$ have been left out. This clearly shows the importance of these terms for obtaining the correct average deformation energies.

In fig. 6 we present a microscopical test of the semiclassical results. The corresponding $\mathrm{HF}$ calculations were done in ref. [7]. The figure shows the full HF result with 2 minima and 2 maxima. Also shown is the selfconsistently Strutinsky-smoothed HF energy, calculated as discussed in section 2.3. The semiclassical ETF result is shown by the dashed line (adjusted at $Q=0$ ). The agreement of the two average curves is better than $1 \mathrm{MeV}$ at all deformations included. This gives once again a nice

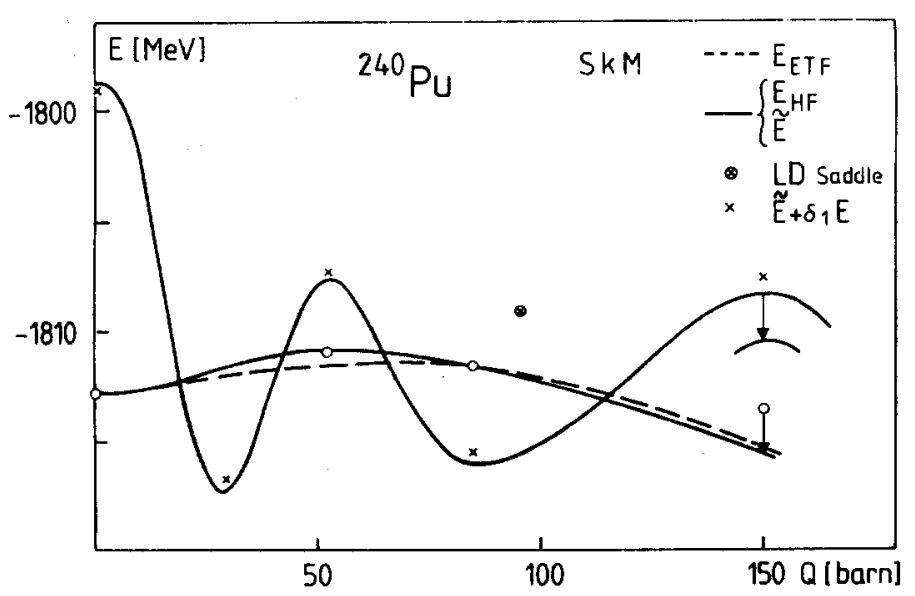

Fig. 6. Total energy of ${ }^{210} \mathrm{Pu}$ versus mass quadrupole moment $Q$ obtained with the SkM force. Solid lines: microscopical energy with shell eflects $\left(E_{\mathrm{HF}}\right)$ and selfconsistently Strutinsky-averaged energy $(\tilde{E})$; both calculated with the constrained HF method (see ref. [7] for details). Crosses indicate the "Strutinsky approximation" $E+\delta_{1} E$. Dashed line: variational ETF result (as in figs. 4 and 5). The encircled cross indicates the empirical LDM saddle point. 
confirmation of the semiclassical method. It shows in particular also that the slight overbinding of the ETF results discussed in section 4.2 above $(\sim 8 \mathrm{MeV}$ in this nucleus) does not affect the deformation energies noticeably. The crosses in fig. 6 show the results obtained after adding the shell-correction energy $\delta_{1} E$ to the average curves; they reproduce the exact $\mathrm{HF}$ values within less than $0.5 \mathrm{MeV}$.

Encouraged by these results, we are thus able to make reliable predictions of fission barriers using the semiclassical method. By a simple readjustment of the Skyrme parameters, which we will discuss in the next paragraph, we have constructed the force $\mathrm{SkM}^{*}$ which gives the correct empirical barrier height of ${ }^{240} \mathrm{Pu}$, see fig. 4 .

A few words may be said about the number of density variational parameters included in the calculations for deformed shapes. Since we have seen in the spherical case that the building-up of a slight dip in the proton densities (due to the Coulomb repulsion) lowers the energy only by $0.2 \mathrm{MeV}$ in ${ }^{208} \mathrm{~Pb}$, we have restricted the deformation energy calculations to the six variational parameters $\rho_{0 \mathrm{q}}, \alpha_{\mathrm{q}}$, $\gamma_{\mathrm{q}}$ defined in eq. (4.1) (imposing $\rho_{1 \mathrm{q}}=0$ ). An interesting result was that even these parameters vary very little with deformation; this is demonstrated for a few points along the fission barrier in table 6 . The quantity $\Delta E$ gives the gain in energy brought about at each deformation by the readjustment of the parameters $\rho_{0 \mathrm{q}}, \alpha_{\mathrm{q}}$ and $\gamma_{\mathrm{q}}$. This shows us that - for the realistic force $\mathrm{SkM}^{*}$ - one would only make an error of $\sim 0.5 \mathrm{MeV}$ in the barrier height if one would use constant density parameters.

Table 6

\begin{tabular}{|c|c|c|c|c|c|c|c|}
\hline$C$ & $\rho_{0 p}$ & $\rho_{0 n}$ & $\alpha_{p}$ & $\alpha_{\mathrm{n}}$ & $\gamma_{p}$ & $\gamma_{n}$ & $\Delta E$ \\
\hline 1.0 & 0.0618 & 0.0890 & 0.571 & 0.634 & 1.46 & 1.45 & 0.0 \\
\hline 1.4 & 0.0629 & 0.0914 & 0.570 & 0.641 & 1.45 & 1.44 & -0.19 \\
\hline 1.6 & 0.0634 & 0.0907 & 0.566 & 0.637 & 1.45 & 1.44 & -0.58 \\
\hline 1.8 & 0.0639 & 0.0910 & 0.566 & 0.637 & 1.45 & 1.44 & -1.65 \\
\hline
\end{tabular}

The asymmetry of the surface profiles governed by the parameters $\gamma_{\mathrm{q}}$ was shown in section 4.2 to lower the binding energy of ${ }^{208} \mathrm{~Pb}$ by about $5 \mathrm{MeV}$. Its influence on the fission barrier is shown in fig. 7, where the barrier of ${ }^{240} \mathrm{Pu}$ has been calculated once with $\gamma_{\mathrm{q}}=1$ and once with the variational values $\gamma_{q} \neq 1$. The difference is seen to be $\sim-0.8 \mathrm{MeV}$ at the saddle, corresponding to a decrease of $\sim 0.3 \mathrm{MeV}$ of the surface energy (see section 5.2). This difference is typical for forces which give approximately correct average fission barriers [72]. We also show in fig. 7 the results obtained for the same force $\mathrm{SkM}^{*}$, but with the simplified functional $\tau[\rho]$ of ref. [61] where $\tau_{4}$ was omitted and the Weizsäcker coefficient was multiplied by two. This leads to an overestimation of the barrier height, as can be expected on the basis of our discussion in section 3.2. We saw there that an increase of the Weizsäcker term by a factor of $\sim 1.4$ might lead to realistic deformation energies; however, the density profiles then have unrealistically steep tails and cannot fit electron scattering data. This is also reflected in the larger gain obtained due to the surface asymmetry parameters $\gamma_{\mathrm{q}} \neq 1$ seen in fig. 7; in fact, the variational values found in this case were $\gamma_{\mathrm{p}} \simeq 3.2$ and $\gamma_{\mathrm{n}} \simeq 2.3$. The situation is even worse if the MTF functional of ref. [59] (in which the Weizsäcker term is multipled by $\sim 4$ ) is used for a fission barrier calculation; we find with it a barrier height for ${ }^{240} \mathrm{Pu}$ of over $30 \mathrm{MeV}$.

In the calculations to fig. 7 (and to fig. 8 below) we have, for reasons of computer times, calculated the Coulomb energy only in the LDM approximation. As shown in appendix D, this leads to an overestimation of the barrier of ${ }^{240} \mathrm{Pu}$ by $\sim 1.2 \mathrm{MeV}$ compared to an exact integration of the diffuse 


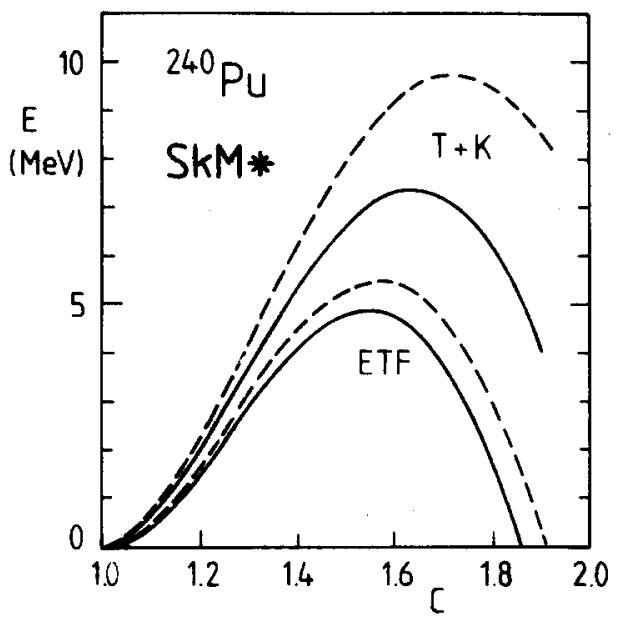

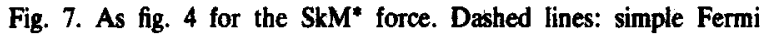
function density profiles $\left(\gamma_{\mathrm{q}}=1\right)$; solid lines: asymmetric profiles $\left(\gamma_{\mathrm{q}} \neq 1\right)$. Lower curves (ETF): using the full ETF functionals up to 4th order. Upper curves $(T+K)$ : using the truncated phenomenological functional $\tau[\rho]$ of Treiner and Krivine [61]. (Coulomb diffuseness correction not included.)

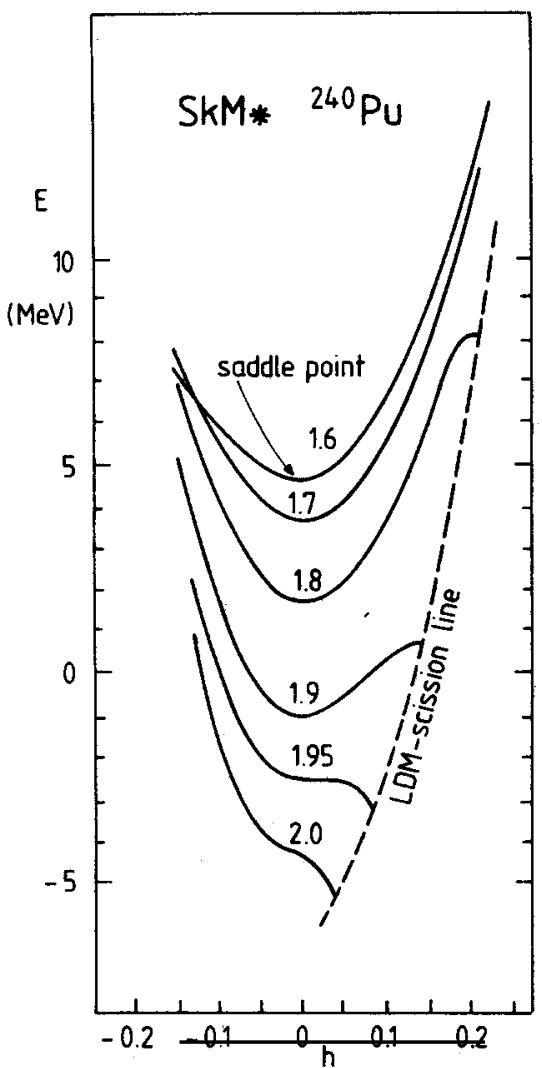

Fig. 8. Cuts through the ETF deformation energy surface in the $(c, h)$ parametrisation [7] of ${ }^{200} \mathrm{Pu}$, obtained with the $\mathbf{S k M}^{*}$ force. The energy is plotted versus the neck parameter $h$ for different elongations $c$ (given by numbers). Only the region beyond the saddle point $(c=1.6)$ is shown. Note the disappearance of the scission barrier around $c \approx 1.95$.

proton density. This error is sufficiently small so that it does not affect the differences between the curves shown in fig. 7.

An important feature of the LDM calculations for fission is the prediction of a well-defined so-called "exit point" [83] in the deformation energy surface at which the ridge, which separates the fission valley from the fusion valley, disappears and which can be interpreted as the physical scission point. In the LDM calculations of ref. [83] an unrestricted variation of deformations of the liquid drop was carried out, only imposing the centre-of-mass distance $2 \rho$ between the two forming fragments. The exit point was characterized by a critical value $\rho_{\text {crit }} \approx 1.17$ (in units of the spherical radius $R_{0}$ ). For shapes with $\rho>\rho_{\text {crit }}$, there was no other extremum of the energy than the minimum lying in the fusion valley, corresponding to separated fragments. The radius of the neck at the exit point was found to be $r_{\mathrm{n}} \sim 0.27 R_{0}$. Both these values, $\rho_{\text {crit }}$ and $r_{\mathrm{n}}$ in units of $\boldsymbol{R}_{0}$, were almost independent of the fissility parameter $X=E_{\mathrm{c}}^{(0)} / 2 E_{\mathrm{s}}^{(0)}$, i.e. the ratio of the Coulomb to twice the surface energy of the spherical nucleus. (See ref. [122] for a recent analysis of experimental information on the exit point shapes.)

These general trends were later confirmed in the LDM calculations with the restricted $(c, h)$ shape parametrisation [37]. It may be interesting to see whether these features persist in an extended 
semiclassical treatment which takes the surface diffuseness into account, such as the variational ETF model.

In fig. 8 we show cuts through the ETF deformation energy surface of ${ }^{240} \mathrm{Pu}$ obtained with the force $\mathrm{SkM}^{*}$. For fixed values of the elongation parameter $c$ (indicated by the numbers), the energies are plotted versus the neck parameter $h$. The LDM scission line (corresponding to the sharp surface) is indicated by the dashed line, beyond which the energy drops down steeply towards the fusion valley, thus leading to scission. The disappearance of the stabilizing ridge against scission is clearly seen for $c \simeq 1$.9. Converting the $(c, h)$ surface into a $(\rho, h)$ plot, where $\rho$ is the fragment's centre-of-mass distance, an analogous picture is obtained with an exit point at $\rho_{\text {crit }} \simeq 1.2 R_{0}$ and a neck radius of the half-density surface $r_{\mathrm{n}} \simeq 2 \mathrm{fm} \simeq 0.28 R_{0}$, in fair agreement with the old LDM result [83]. The energy at the exit point lies below the energy of the spherical shape by $\sim 3 \mathrm{MeV}$ in the $(c, h)$ plane and by $\sim 9 \mathrm{MeV}$ in the $(\rho, h)$ plane, in good agreement with the results in ref. [37] (see in particular figs. VII-2 and VII-3 there). The geometry of this exit point (given by $\rho_{\text {crit }}$ and $r_{n}$ in $R_{0}$ ) turns out to be rather independent of the force used, as can be seen from the result in ref. [68] where the force SIII was used, which gave a far too high barrier corresponding to a too high surface energy. This is thus equivalent to the near independence of $\rho_{\text {crit }}$ and $r_{n}$ of the fissility $X$ found in the LDM calculations [83]. The energy of the exit point, however, depends crucially on the height of the fission barrier. Therefore, reliable predictions about the energy difference between the ground state and the exit point can only be made if the force employed reproduces the empirical saddle point energy.

We may thus conclude that a selfconsistent semiclassical calculation with diffuse densities, using an effective force which is adjusted to reproduce the LDM saddle point, gives almost quantitatively the same results concerning the exit point as the LDM with sharp density surfaces. This is even more surprising if one realizes that our semiclassical model is more refined than the LDM not only because of the diffuse density profiles, but also in the sense that it automatically contains curvature energy, surface and curvature asymmetry and even higher order contributions which go beyond the simple LDM used in refs. [37,83]. It appears that the existence and even the location of the exit point depends only little on those refinements.

One should note that the nuclear shape at the exit point is rather compact and still little constricted with a neck diameter of $\sim 4 \mathrm{fm}$. This seems to justify our assumptions of a saturated central density and a constant diffuseness parameter $\alpha$. In going beyond these deformations - which would have to be done in the framework of a dynamical model since the rupture of the neck is presumably a rather fast, non-adiabatic process - our way of treating the variational problem using parametrized trial densities might not easily be extended, and the use of a finite grid with the densities themselves as variational parameters might become more appropriate.

\subsection{Adjustment of the force $S k M^{*}$}

The main goal of adjusting the Skyrme force SkM*, which we have been mainly using, was to solve the longstanding problem of the fission barriers mentioned in the introduction. Since the constrained HF calculations would have been too time consuming for a readjustment of the Skyrme parameters, it was essential to know that the variational ETF calculations allow to predict quantitatively the average fission barriers for a given Skyrme force with a computational effort at least two orders of magnitude smaller.

In earlier semiclassical calculations [69] we found that the SkM force [34] was a good candidate, giving an appreciably lower fission barrier for ${ }^{240} \mathrm{Pu}$ than any of the older Skyrme forces. After the 
improvement of the parametrisation of deformed densities discussed in the last sections, it turned out that, in fact, the barrier for SkM was somewhat too low, see fig. 4. At the same time HF calculations with the SkM force [7] revealed that it leads to a systematic overestimation of the binding energies by an amount which is roughly proportional to $A^{2 / 3}$. This, as well as the too low fission barrier, can be understood as a defect of the surface energy as inherent in the force SkM; using LDM estimates of the binding energy and the fission barrier height, both defects can in fact be explained by a lack of surface energy of $\sim 0.6-0.7 \mathrm{MeV}$.

In section 5 we shall see how the surface energy $a_{\text {s }}$ can be calculated in terms of the Skyrme parameters. It depends rather crucially on the coefficient $b$ of the term $b(\nabla \rho)^{2}$ in the energy density, which is given by (for $\rho_{\mathrm{n}}=\rho_{\mathrm{p}}$ )

$$
b=\frac{1}{64}\left(9 t_{1}-5 t_{2}\right)
$$

in fact, about one half of the surface energy $a_{\mathrm{s}}$ comes from this contribution (see eq. (6.70) and the discussion in section 6.5).

In attempting to increase the surface energy of the force SkM, we wanted of course to keep its good properties, in particular $K_{\infty}$ and $m_{\infty}^{*}$ which were adjusted originally in fitting the force SkM to the breathing mode and giant quadrupole energies by RPA type calculations [34]. By inspecting eqs. (2.12)-(2.17) we see that the nuclear matter properties do not depend on $t_{1}$ and $t_{2}$. It is thus possible to change $b$ (eq. (4.11)) and with it the surface energy $a_{s}$, without affecting $m_{\infty}^{*}$ or any other of the nuclear matter properties.

It is thus by varying $t_{1}$ and $t_{2}$ such as to increase $b$ (eq. (4.11)) and to keep $\beta$ (eq. (2.14)) constant, that the force SkM* was adjusted to give the empirical LDM barrier height of ${ }^{240} \mathrm{Pu}$, as shown in fig. 4 . That the HF results [7] for the binding energies and radii of stable spherical nuclei would then come to such a nice detailed agreement with experiment as seen in tables 4 and 5 , could of course not be expected and is very fortunate.

The $\mathrm{SkM}^{*}$ force gives less good binding energies for unstable nuclei far away from the $\beta$-stability line, as seen in table 4 . This indicates that it still does not have the correct asymmetry properties. There is, however, a good chance to improve on this. Of the four asymmetry force parameters $x_{1}, x_{2}, x_{3}$ and $x_{0}$, only the last one was used in the fit of SkM and SkM*, the three others were kept equal to zero. By playing on these parameters it should be possible to improve the asymmetry properties. An analysis along these lines is actually in progress [72].

\subsection{Perturbative inclusion of shell effects}

Two practical ways of including the shell effects shall briefly be discussed here. The first one is evidently to use the Strutinsky shell-corrections $\delta_{1} E_{\mathrm{q}}$ defined in eq. (2.26); they are obtained by solving the Schrödinger equation (2.27) using the mean Skyrme fields $V_{\mathrm{q}}\left[\rho_{\mathrm{q}}\right], m_{\mathrm{q}}^{*}\left[\rho_{\mathrm{q}}\right]$ and $\boldsymbol{W}_{\mathrm{q}}\left[\rho_{\mathrm{q}}\right]$ (eqs. (2.9-2.11)), calculated from the variational semiclassical densities $\rho_{\mathrm{q}}$ using the functionals $\tau_{\mathrm{ETF}}[\rho]$ and $J_{E T F}[\rho]$. The disadvantage of this method is that the final result

$$
E=E_{\mathrm{ETF}}+\delta_{1} E_{\mathrm{n}}+\delta_{1} E_{\mathrm{p}}
$$

will suffer from the same overbinding defect as the ETF energies which we discussed in section 4.2 above. 
The second way, which takes only a little more computational effort, is to employ the "expectation value method" (EVM) developed earlier [74, 84] and successfully used as an approximation to the HF method $[58,84,85]$. It consists in constructing Slater determinants $\hat{\phi}\left[\rho_{\mathrm{ETF}}\right]$ out of the eigenfunctions $\hat{\varphi}_{\nu}^{\mathrm{q}}$ of the variational ETF fields $V_{\mathrm{q}}(\boldsymbol{r}), m_{\mathrm{q}}^{*}(\boldsymbol{r})$ and $\boldsymbol{W}_{\mathrm{q}}(\boldsymbol{r})$ obtained as above, and calculating the eigenvalue of the total two-body Skyrme Hamiltonian with respect to $\hat{\phi}\left[\rho_{\mathrm{ETF}}\right]$ :

$$
E_{\mathrm{EVM}}=\left\langle\hat{\phi}\left[\rho_{\mathrm{ETF}}\right]\left|\hat{T}+\mathscr{V}_{\mathrm{Sky}}\right| \hat{\phi}\left[\rho_{\mathrm{ETF}}\right]\right\rangle
$$

In other words: one performs one HF iteration with the semiclassical mean fields as an input.

The last column of table 4 above shows the results for the binding energies $B^{\mathrm{ETF}}$ obtained in this way for some spherical nuclei. They are smaller (corresponding to the Ritz variational principle) than the HF energies $B^{\mathrm{HF}}$ by less than $\sim 1 \mathrm{MeV}$ in light and less than $\sim 3 \mathrm{MeV}$ in heavy nuclei. This demonstrates that the EVM allows to obtain rather accurate total energies including shell effects at the cost of essentially one microscopical HF step beyond the semiclassical calculation. Of course, other quantities depending on the $\hat{\varphi}_{\nu}^{q}$ such as r.m.s. radii or multipole moments can be calculated as well and should lead to similarly good approximations to purely microscopical HF results.

As a further illustration we show in fig. 9 the neutron and proton density distributions of ${ }^{208} \mathrm{~Pb}$ obtained in the EVM approximation (dashed line), and compare them to the selfconsistent HF results. The agreement is seen to be very satisfactory; the small differences of $\sim 1-2 \%$ in the inner region are certainly smaller than the differences between the HF and the experimental densities.

We have thus demonstrated that the variational ETF method with perturbative inclusion of the shell effects by one HF iteration provides an excellent approximation to purely microscopical HF calculations. It is therefore certainly an economical tool for calculating fission barriers including shell effects. Based on the test shown in fig. 6 above, we can expect the relative deformation energies to be within $\sim 1 \mathrm{MeV}$ of what one would obtain in a-much more lengthy-HF calculation using the corresponding constraints.

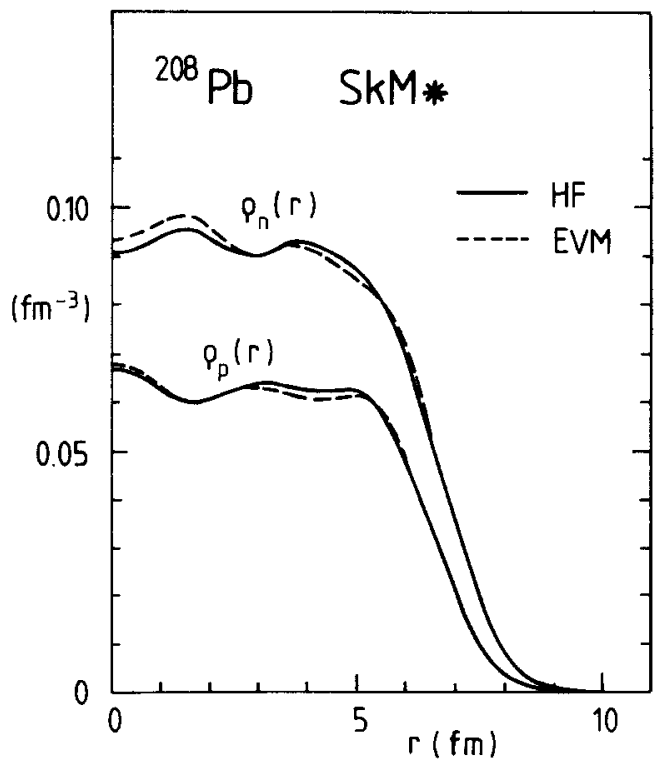

Fig. 9. Density profiles of ${ }^{208} \mathrm{~Pb}$ as in fig. 1. The dashed curves include the shell effects perturbatively using the expectation value method (EVM) on top of the ETF calculation. 
In the last step of this combined ETF plus EVM method, any small systematic errors in the ETF energies (e.g. the slight overbinding effect discussed above) are automatically corrected in perturbation. Therefore, several simplifications might be admissible in the ETF calculation of deformation energies, such as restriction to pure Fermi functions, keeping the parameters of the density profiles constant and using the LDM (i.e. the sharp surface) approximation to the Coulomb energy.

We should emphasize once more that we have not included any corrections due to spurious rotational energies. In the ETF model they turn out to be zero, as discussed in the appendix B. In HF calculations they are, however, known to affect deformation energies appreciably $[1,5]-$ as well as vibrational zero point energies $[79,80]$ - and should therefore be added at the end of a ETF + EVM calculation. In this respect, the force $\mathrm{SkM}^{*}$ should be readjusted under inclusion of the corresponding corrections, which could certainly be done by increasing the surface energy somewhat more. Rather than doing this, we took the phenomenological point of view of thinking these corrections to be renormalized into the effective force-as this is done anyhow with other (e.g. RPA) ground-state correlations when fitting HF energies to experimental binding energies.

\section{Liquid drop model type expansion of the ETF binding energy}

In this section we shall make quantitative connections between the parameters of the Skyrme forces and those of phenomenological macroscopic models such as the liquid drop model (LDM) [82] and the droplet model [19]. The basic idea of these models is to expand the nuclear binding energy in powers of the inverse radius $R$, i.e. in powers of $A^{-1 / 3}$. To obtain such an expansion one profits from the fact that for not too small nuclei, the density distributions are "leptodermous", i.e. they have a surface thickness $\alpha$ which is much smaller than the bulk radius

$$
\alpha \ll R
$$

so that it is legitimate to expand in powers of $\alpha / R$. In the droplet model, one further expands in powers of other small quantities in order to take into account compression and asymmetry effects (see section 5.3). Our semiclassical model gives an ideal starting point to such an expansion. As we have seen in section 4, nucleon densities with asymmetric Fermi type profiles minimize the total ETF energies within a few hundred $\mathrm{keV}$; the energies obtained hereby are very close to the average HF energies.

We have recently adapted in a systematic way the leptodermous expansion to the Skyrme ETF formalism [86]. We shall in the following outline it for symmetric nuclei without Coulomb interaction $\left(\rho_{\mathrm{p}}=\rho_{\mathrm{n}}=\frac{1}{2} \rho\right)$. Asymmetry properties will be dealt with in the limit for semi-infinite nuclear matter in section 5.2, and the leptodermous expansion of the Coulomb energy is discussed in appendix D.

\subsection{Leptodermous expansion of the energy for symmetric nuclei}

We now show how to expand the exact expression for the total nuclear energy, written in the $(u, v)$ coordinates discussed in appendix $\mathrm{C}$

$$
E=\int \mathrm{d}^{3} r \mathscr{E}[\rho(r)]=2 \pi \int_{v_{1}}^{v_{2}} \mathrm{~d} v \int_{-R_{2}}^{\infty} g(u, v) \mathscr{E}[\rho(u)] \mathrm{d} u
$$


in the leptodermous approximation, i.e. in the limit $R_{2} \gg \alpha$. This approximation should be well fulfilled for heavy nuclei up to typical saddle point deformations (or even beyond) where the actinide nuclei have almost no neck. The density profile across the LDM surface shall be given by

$$
\rho(u)=\rho_{0} /[1+\exp (u / \alpha)]^{\gamma} .
$$

For $R_{2} \gg \alpha$, the lower limit of the $u$ integration in eq. (5.2) (which, in fact, is the only reason for the integral not to separate in $u$ and $v$ !) can be practically replaced by $-\infty$ for all contributions except the one which gives the volume energy. We therefore separate the latter out in writing

$$
E=a_{\mathrm{v}} A+\Delta E, \quad a_{\mathrm{v}}=\mathscr{E}\left[\rho_{0}\right] / \rho_{0}=e_{\infty}\left(\rho_{0}\right)
$$

The correction $\Delta E$ is then exactly

$$
\Delta E=2 \pi \int_{v_{1}}^{v_{2}} \mathrm{~d} v \int_{-R_{2}}^{\infty} \mathrm{d} u g(u, v)\left\{\mathscr{E}[\rho(u)]-a_{v} \rho(u)\right\}
$$

The leptodermous approximation (to be checked numerically below) consists in writing $E$, which contains only contributions near the surface, i.e. in a region $-\alpha \leq u \leq+\alpha$, as

$$
\Delta E \simeq 2 \pi \int_{v_{1}}^{v_{2}} \mathrm{~d} v \int_{-\infty}^{+\infty} \mathrm{d} u g(u, v)\left\{\mathscr{E}[\rho(u)]-a_{\mathrm{v}} \rho(u)\right\}
$$

Since the integral now separates, we obtain with eqs. (C.8-11) in terms of the surface $S$ and the mean curvature $C$ of the generating LDM surface

$$
\Delta E \simeq b_{0} S+b_{1} \cdot 2 C+b_{2} \cdot 4 \pi=\Delta E_{\text {lept. }}
$$

where the coefficients $b_{0}, b_{1}$ and $b_{2}$ can be obtained once for all for a given energy density as functions of $\rho_{0}, \alpha, \gamma$ and the force parameters.

The explicit expressions for the coefficients $b_{n}$ are given in the following. Because of the explicit $v$ dependence (through $R_{1}, R_{2}$ ) of the Laplacian $\Delta \rho$ (see eq. (C.13)) we have to split up the total energy density. Writing

$$
\mathscr{E}[\rho]=\mathscr{F}(u)+\mathscr{G}(u) \Delta \rho+\mathscr{H}(u)[\Delta \rho]^{2},
$$

where the functions $\mathscr{F}, \mathscr{G}$ and $\mathscr{H}$ may only depend on $\rho$ and $(\nabla \rho)^{2}$, one gets*

\footnotetext{
* Due to a copying error, the expressions for $b_{1}$ and $b_{2}$ were given wrongly in ref. [86].
} 


$$
\begin{aligned}
& b_{0}=\int_{-\infty}^{+\infty} \hat{\mathscr{F}}(u) \mathrm{d} u \\
& b_{1}=\int_{+\infty}^{+\infty}\left\{u \hat{\mathscr{F}}(u)+\mathscr{G}(u) \rho^{\prime}(u)+2 \mathscr{H}(u) \rho^{\prime}(u) \rho^{\prime \prime}(u)\right\} \mathrm{d} u \\
& b_{2}=\int_{-\infty}^{+\infty}\left\{u^{2} \hat{\mathscr{F}}(u)+2 u \rho^{\prime}(u)\left[\mathscr{G}(u)+2 \mathscr{H}(u) \rho^{\prime \prime}(u)\right]+4 \mathscr{H}(u)\left[\rho^{\prime}(u)\right]^{2}\right\} \mathrm{d} u \\
& \hat{\mathscr{F}}(u)=\mathscr{F}(u)+\mathscr{G}(u) \rho^{\prime \prime}(u)+\mathscr{H}(u)\left[\rho^{\prime \prime}(u)\right]^{2}-a_{\mathrm{v}} \rho(u)
\end{aligned}
$$

In eqs. (5.9), the primes on $\rho$ denote derivation with respect to $u$. In arriving at this result, we have neglected a term

$$
2 \pi \int_{v_{1}}^{v_{2}} \mathrm{~d} v \frac{1}{R_{1}} \int_{-R_{2}}^{\infty} \mathrm{d} u \mathscr{H}(u)\left[\rho^{\prime}(u)\right]^{2}\left(\frac{\boldsymbol{R}_{1}-\boldsymbol{R}_{2}}{u+R_{2}}+\frac{\boldsymbol{R}_{2}-\boldsymbol{R}_{1}}{u+R_{1}}\right)
$$

whose deformation dependence is more complicated than that of the above simple invariants $C, S$ or a constant. However, this term is seen to be exactly zero in the spherical case and negligibly small otherwise.

The coefficients $b_{n}$ (eq. (5.9)) depend on the density parameters $\rho_{0}, \alpha$ and $\gamma$. To the extent that these depend only negligibly on the deformation - as we have found it in section 4.3 to be the case, at least up to saddle point deformations in heavy nuclei-the entire deformation dependence of the energy is contained in the quantities $S$ and $C$.

We have checked the validity of the leptodermous approximation $\Delta E_{\text {lept }}$ (eq. (5.7)) numerically. Figure 10 shows for the case $N=Z=120$ the error

$$
\Delta E_{\text {non-lept }}=\Delta E_{\text {lept }}-\Delta E_{\text {exact }} \text {, }
$$

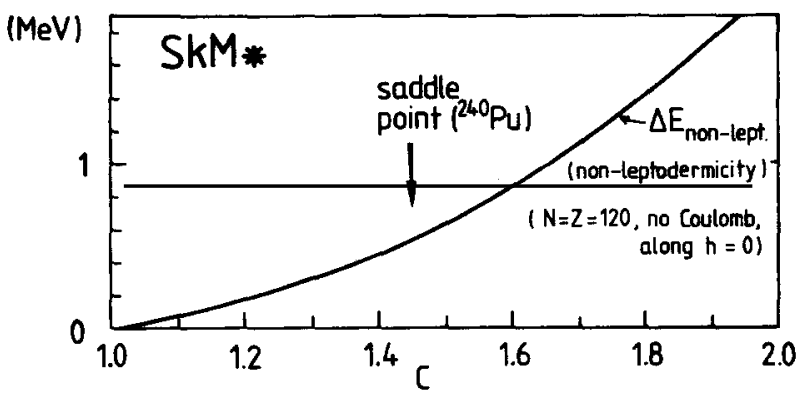

Fig. 10. Error $\Delta E_{\text {non-lept }}$ (eq. (5.11)) of the leptodermous approximation to the total energy of a heavy symmetric nucleus (without Coulomb interaction) versus elongation $c$. 
where $\Delta E_{\text {exact }}$ is the exactly integrated quantity (eq. (5.5)). The error is seen to be surprisingly small even at deformations corresponding to the exit point region of actinide nuclei (see the discussion at the end of section 4.3); at the typical saddle point deformations $(c \approx 1.4-1.5)$, it is $\sim 0.5 \mathrm{MeV}$. Note that for the spherical shape, the error of the leptodermous approximation is less than $0.01 \mathrm{MeV}$ and thus completely negligible! (This shows that the main error in $\Delta E_{\text {lept }}$ comes from neglecting the quantity in eq. (5.10).)

We now go on to sort out the dependence of the energy on the nucleon number. Its leptodermous expansion is given in appendix $\mathrm{C}$ :

$$
A=\int \mathrm{d}^{3} r \rho(r)=\rho_{0}\left[V+c_{0} \alpha S+2 c_{1} \alpha^{2} C+4 \pi c_{2} \alpha^{3}\right]+\mathcal{O}\left(\exp \left(-R_{2} / \alpha\right)\right)
$$

where the $c_{n}$ are constants which only depend on the parameter $\gamma$ of eq. (5.3). The contributions of order $\exp \left(-R_{2} / \alpha\right)$ are practically negligible for not too light nuclei $(A \geq 20)$. In the spherical case we have $V=(4 \pi / 3) R_{0}^{3}, S=4 \pi R_{0}^{2}$ and $C=4 \pi R_{0}$, where $R_{0}$ is the "sharp" (or half-density) radius which is adjusted to keep $A$ constant. For the deformed case we introduce in the usual manner the shape functions $B_{\mathrm{s}}$ and $B_{\mathrm{c}}$, which are normalized to unity for the spherical case, by defining

$$
S=4 \pi R_{0}^{2} B_{\mathrm{s}} ; \quad C=4 \pi R_{0} B_{\mathrm{c}}
$$

with which we get

$$
A \simeq \frac{4 \pi}{3} \rho_{0} R_{0}^{3}\left\{1+3 c_{0} B_{\mathrm{s}}\left(\frac{\alpha}{R_{0}}\right)+6 c_{1} B_{\mathrm{c}}\left(\frac{\alpha}{R_{0}}\right)^{2}+3 c_{2}\left(\frac{\alpha}{R_{0}}\right)^{3}\right\}
$$

This equation can be inverted to obtain the following expansion for $\boldsymbol{R}_{0}$

$$
R_{0}=r_{0} A^{1 / 3}-c_{0} \alpha B_{\mathrm{s}}+\left(c_{0}^{2} B_{\mathrm{s}}^{2}-2 c_{1} B_{\mathrm{c}}\right) \frac{\alpha^{2}}{r_{0}} A^{-1 / 3}+\cdots
$$

with

$$
r_{0}=\left(3 / 4 \pi \rho_{0}\right)^{1 / 3}
$$

For heavy nuclei $(A \geq 200)$ one can verify, using realistic values of $\alpha(\sim 0.5)$ and $\gamma(\leq 2)$, that the first three terms in eq. (5.15) give the correct numerical value of $R_{0}$ within less than $10^{-3}$, even at very large deformations (see ref. [86]). For pure Fermi profiles $(\gamma=1)$ we have $c_{0}=c_{2}=0, c_{1}=\pi^{2} / 6$, and one obtains the familiar expression

$$
R_{0}=r_{0} A^{1 / 3}-\frac{\pi^{2}}{3} \frac{\alpha^{2}}{r_{0}} B_{c} A^{-1 / 3}
$$

We now insert the expansion of $R_{0}$ (eq. (5.15)) into eq. (5.13) and obtain with eqs. $(5.4,5.7)$ the leptodermous expansion of the total energy 


$$
E=a_{\mathrm{v}} A+a_{\mathrm{s}} A^{2 / 3}+a_{\mathrm{c}} A^{1 / 3}+a_{0}+\mathcal{O}\left(A^{-1 / 3}\right)
$$

where $a_{\mathrm{v}}$ is defined in eq. (5.4) and

$$
\begin{aligned}
& a_{\mathrm{s}}=4 \pi r_{0}^{2} b_{0} B_{\mathrm{s}}, \\
& a_{\mathrm{c}}=8 \pi r_{0}\left(b_{1} B_{\mathrm{c}}-b_{0} c_{0} \alpha B_{\mathrm{s}}^{2}\right), \\
& a_{0}=4 \pi b_{2}-8 \pi c_{0} \alpha b_{1} B_{\mathrm{s}} B_{\mathrm{c}}+4 \pi b_{0} \alpha^{2} B_{\mathrm{s}}\left(3 c_{0}^{2} B_{\mathrm{s}}^{2}-4 c_{1} B_{\mathrm{c}}\right) .
\end{aligned}
$$

The terms of order $A^{-1 / 3}$ and lower contribute less than $1 \mathrm{MeV}$ to the total energy of heavy nuclei and are thus practically unimportant. The deformation dependence of the energy (5.18) is entirely contained in the shape functions $B_{\mathrm{s}}$ and $B_{\mathrm{c}}$. The main $A$ dependence of the energy is also separated out in eq. (5.18); a very smooth variation is, however, still coming from the $b_{n}$ and $r_{0}$ through their dependence on $\rho_{0}, \gamma$ and $\alpha$ which are determined for each nucleus by the variational equation (1.1). This dependence cannot be determined analytically in the general case. When pure Fermi functions are used for the densities $(\gamma=1)$, the analytical dependence of $E$ (eq. (5.18)) can be worked out (the result for the surface energy $a_{\mathrm{s}}$ is given in ref. [16]); it is however too complicated for doing the variation eq. (1.1) analytically.

We can come one step further by neglecting the variation of $\alpha$ and $\gamma$ with $A$ and only determining $\rho_{0}(A)$, to which the energy is most sensitive. Proceeding as in the development of the droplet model [19], we expand $E$ in powers of the small quantity

$$
\varepsilon=-\frac{1}{3}\left(\rho_{0}-\rho_{\infty}\right) / \rho_{\infty}
$$

Expanding the first three terms in eq. (5.18) - the constant term $a_{0}$ will be seen below to be unimportant in this context - in powers of $\varepsilon$, we write

$$
\begin{aligned}
& a_{\mathrm{v}}=a_{\mathrm{v}}^{\infty}+\frac{1}{2} K_{\infty} \varepsilon^{2}-\frac{9}{2} \ddot{a}_{\mathrm{v}} \varepsilon^{3}, \\
& a_{\mathrm{s}}=a_{\mathrm{s}}^{\infty}-3 \dot{a}_{\mathrm{s}} \varepsilon+\frac{9}{2} \ddot{a}_{\mathrm{s}} \varepsilon^{2}, \\
& a_{\mathrm{c}}=a_{\mathrm{c}}^{\infty}-3 \dot{a}_{\mathrm{c}} \varepsilon+\frac{9}{2} \ddot{a}_{\mathrm{c}} \varepsilon^{2}
\end{aligned}
$$

Hereby we have used the dots for denoting reduced derivatives with respect to $\rho_{0}$,

$$
\dot{a}_{\mathrm{s}}=\left.\rho_{\infty} \frac{\mathrm{d} a_{\mathrm{s}}}{\mathrm{d} \rho_{0}}\right|_{\rho_{\infty}}, \quad \ddot{a}_{\mathrm{s}}=\left.\rho_{\infty}^{2} \frac{\mathrm{d}^{2} a_{\mathrm{s}}}{\mathrm{d} \rho_{0}^{2}}\right|_{\rho_{\infty}},
$$

etc. In eq. (5.23) $a_{\mathrm{v}}^{\infty}$ and $K_{\infty}$ are the infinite nuclear matter binding energy and incompressibility given by eqs. $(2.12,2.16)$. In eqs. $(5.24,5.25) a_{\mathrm{s}}^{\infty}$ and $a_{\mathrm{c}}^{\infty}$ are the surface and curvature energies, which will be evaluated in the limit of semi-infinite nuclear matter in the next section. They as well as their derivatives $\dot{a}_{\mathrm{s}}$ etc. depend on $\alpha$ and $\gamma$. Inserting the expansions (5.23-5.25) into $E$ (eq. (5.18)), we find from the stationary condition $\mathrm{d} E / \mathrm{d} \varepsilon=0$ the following expression [86] ${ }^{*}$

* Neglecting the cubic term in eq. (5.23), since $\bar{a}_{\mathrm{v}}$ is rather small (cf. table 2). 


$$
\varepsilon(A)=\frac{\left(\dot{a}_{\mathrm{s}} A^{-1 / 3}+\dot{a}_{\mathrm{c}} A^{-2 / 3}\right)}{\left(K_{\infty} / 3+3 \ddot{a}_{\mathrm{s}} A^{-1 / 3}+3 \ddot{a}_{\mathrm{c}} A^{-2 / 3}\right)}
$$

The result (eq. (5.27)) for $\varepsilon(A)$ can now be inserted into eqs. (5.23-5.25) and with those the full $A$ dependence of the energy $E$ (eq. (5.18)) is obtained (assuming $\alpha$ and $\gamma$ constant). This dependence is, however, not a simple one if the analytical form of $\varepsilon(A)$ (eq. (5.27)) cannot be simplified. We may try to expand it in powers of $A^{-1 / 3}$, although the experience (and the numerical result below) shows that this is not allowed, because $A^{-1 / 3}$ in itself is not a small quantity for real finite nuclei. Doing so nevertheless, we would obtain

$$
\varepsilon(A) \simeq \frac{3 \dot{a}_{\mathrm{s}}}{K_{\infty}} A^{-1 / 3}\left[1+\left(\frac{\dot{a}_{\mathrm{c}}}{\dot{a}_{\mathrm{s}}}-\frac{9 \ddot{a}_{\mathrm{s}}}{K_{\infty}}\right) A^{-1 / 3}+\cdots\right]
$$

In the droplet model [19] one only keeps the lowest order term:

$$
\varepsilon_{\mathrm{DM}}=\frac{3 \dot{a}_{\mathrm{s}}}{K_{\infty}} A^{-1 / 3}=-\frac{2 a_{\mathrm{s}}^{\infty}}{K_{\infty}} A^{-1 / 3}
$$

(The second expression in eq. (5.29) is obtained using the relation $3 \dot{a}_{\mathrm{s}}=-2 a_{\mathrm{s}}^{\infty}$ discussed in the next section.)

Using an expansion of the type of eq. (5.28), one finally arrives at the expansion of the energy

$$
E \approx a_{\mathrm{v}}^{\infty} A+a_{\mathrm{s}}^{\infty} A^{2 / 3}+a_{\mathrm{c}}^{*} A^{1 / 3}+a_{0}^{*}+\cdots
$$

in which now the coefficients are independent of $A$ (except very weakly through $\alpha$ and $\gamma$; this dependence can, however be neglected as the following numerical example will show). The first two terms have not changed in going from eq. (5.18) to eq. (5.30); the curvature energy, however, gets a contribution from the leading term of $\varepsilon(A)$, which usually is called the compression energy [19]:

$$
a_{\mathrm{c}}^{*}=a_{\mathrm{c}}^{\infty}-9 \dot{a}_{\mathrm{s}}^{2} / 2 K_{\infty}
$$

Similar contributions to $a_{0}^{*}$ can be obtained analogously from eq. (5.28).*

We have gone through this rather lengthy derivation of the leptodermous expansion of the energy, eq. (5.30), in order to demonstrate which kind of assumptions and expansions have to be made when one starts from a selfconsistent semiclassical model. Evidently, the usefulness of the expansion eq. (5.30) depends on the validity of each of these various steps. We have already, in passing, mentioned that most of them are well justified. The most crucial step, however, is the expansion of the function $\varepsilon(A)$ (eq. (5.27)). At the time of the development of the droplet model, the incompressibility $K_{\infty}$ of nuclear matter was not known and believed to be of the order of $300-400 \mathrm{MeV}$. Today we know it through the measurements of the nuclear breathing mode to be of the order of $\sim 220 \mathrm{MeV}$. It is quite obvious from looking at eq. (5.27) that the magnitude of $K_{\infty}$ has an influence both on the magnitude of $\varepsilon(A)$ and on the quality of its Taylor expansion eq. (5.28).

\footnotetext{
*Note that our definition of $a_{c}^{\infty}$ through eqs. (5.8), (5.9) and (5.20) contains both the "geometrical" and the "dynamical" contributions discussed in ref. [87].
} 
In fig. 11 we discuss the quantity $\varepsilon(A)$ as a function of $A^{-1 / 3}$. Using the realistic Skyrme force SkM*, we have calculated a series of symmetric nuclei $(N=Z)$ without Coulomb interaction. The crosses show the numerical results of the quantity $\varepsilon$ defined by eq. (5.22) using the variational density and making none of the above approximations. The dashed-dotted line shows the droplet model approximation eq. (5.29). We see that it drastically overestimates the magnitude of $\varepsilon$; in medium and light nuclei the error is more than $100 \%$. The solid line shows the result derived in eq. (5.27) above. Hereby we had to make a choice which values of $\alpha$ and $\gamma$ to use upon whom the coefficients $\dot{a}_{\mathrm{s}}, \ddot{a}_{\mathrm{s}}, \dot{a}_{\mathrm{c}}$ and $\ddot{a}_{\mathrm{c}}$ in eq. (5.27) depend smoothly. We chose the values corresponding to $\bar{A} \approx 170$ which lies roughly in the middle of the region of real nuclei in this plot. (The exact choice of this number does not affect the curve $\varepsilon(A)$ very much.) We see that with this, eq. (5.27) gives an almost quantitative agreement with the exact values of $\varepsilon$ over the whole region, down to $A \sim 40$. If we had used the Taylor expanded form of $\varepsilon(A)$ (eq. (5.28)), we would have obtained the dashed line in fig. 11 which quickly becomes bad for medium and lighter nuclei.

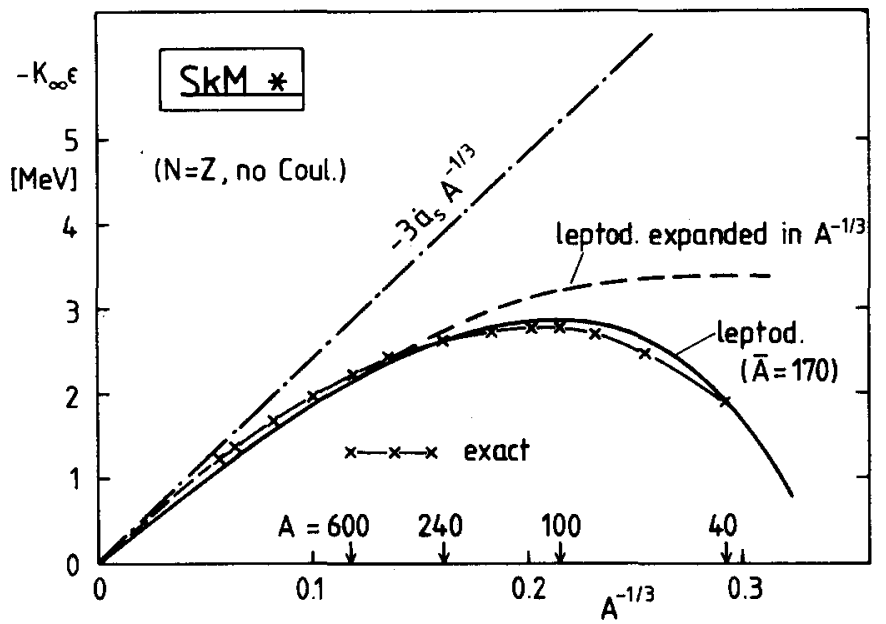

Fig. 11. Compression parameter $\varepsilon$ times $-K_{\infty}$ versus $A^{-1 / 3}$ for symmetric nuclei (no Coulomb). Crosses: exact numerical results of variational ETF calculation. Solid curve: leptodermous expansion result (5.27); dashed curve: Taylor expansion of the latter up to order $A^{-1}$. Dashed-dotted line: droplet model prediction (5.29).

We learn from this that the analytical form of eq. (5.27) is essential and cannot be expanded in powers of $A^{-1 / 3}$, and neither can the total energy be expanded in the form of eq. (5.30). We further learn that the droplet model approximation eq. (5.29) for $\varepsilon(A)$ is very bad, especially for less heavy nuclei, which is essentially due to the low incompressibility of nuclear matter. Indeed, if the same calculation is done with a force with a higher value of $K_{\infty}$, the discrepancy becomes much smaller (see ref. [86]).

From the above derivation it should be rather obvious that its extension to real nuclei with $N \neq Z$ and including the Coulomb interaction would become extremely tedious, if not impossible, if a reliable expansion of the energy has to be obtained. We shall turn back to the discussion of the usefulness and the reliability of the leptodermous expansion of the total energy in section 5.3 in connection with a discussion of the droplet model. 


\subsection{Semi-infinite nuclear matter calculations}

In the asymptotic limit of a very heavy nucleus with $A \rightarrow \infty$, the curvature effects in the surface become negligible and the density profile across the surface is that of so-called semi-infinite nuclear matter. This system has a one-dimensional geometry; the density varies only along one axis (say, the $z$-axis) and is constant as a function of $x$ and $y$. One half of the space (say, for $z<0$ ) is filled with saturated nuclear matter, the other half is empty, and in between (around $z=0$ ), a surface profile $\rho(z)$ develops. Since the volume energy of this system diverges, one can only define the surface energy by

$$
a_{\mathrm{s}}=4 \pi r_{0}^{2} \int_{-\infty}^{+\infty}\left\{\mathscr{E}[\rho(z)]-a_{\mathrm{v}} \rho(z)\right\} \mathrm{d} z
$$

where

$$
a_{\mathrm{v}}=\mathscr{E}\left[\rho_{0}\right] / \rho_{0}, \quad r_{0}=\left(\frac{4}{3} \pi \rho_{0}\right)^{-1 / 3},
$$

and $\rho_{0}$ is the limiting value of $\rho(z)$ for $z \rightarrow-\infty$. Applying the variational principle to $a_{\mathrm{s}}$ (eq. (5.32)) with respect to arbitrary changes in the function $\rho(z)$ (keeping its limiting values $\rho_{0}$ and 0 on both sides) leads to an Euler variational equation which is identical to eq. (3.23) with $\lambda$ replaced by $a_{v}$ and the gradients $\nabla$ replaced by one-dimensional derivatives with respect to $z$. Since in this equation the variable $z$ does not appear explicitly, one can integrate the Euler equation once using the substitution $\rho^{\prime}(z) \mathrm{d} z=\mathrm{d} \rho$, which leads to a third order differential equation if the term $D_{4}[\rho]$ in eq. (3.23) is kept. ${ }^{*}$

We have already discussed the asymptotic solutions of the Euler equation in section 3.3 and also mentioned there that it appears practically impossible to solve it numerically. In the following, we shall discuss numerical variational calculations where we have again used parametrizations of the surface profile $\rho(z)$ and minimized the surface energy with respect to the variational parameters.

\subsubsection{Symmetric case}

We shall first treat the symmetric case with $\rho_{\mathrm{n}}=\rho_{\mathrm{p}}=\frac{1}{2} \rho$ (without Coulomb interaction). In order to develop a feeling for the form of the surface profile, we have tried 4 types of functions $\rho(z)$, minimizing in each case the surface energy eq. (5.32) with respect to the variational parameter(s):

1. Fermi function (one parameter):

$$
\rho(z)=\frac{\rho_{\infty}}{1+\exp \left(z / \alpha_{1}\right)}
$$

2. Error function (one parameter):

$$
\rho(z)=\frac{1}{2} \rho_{\infty}\left[1-\operatorname{erf}\left(z / \alpha_{2}\right)\right]
$$

\footnotetext{
* See section 6.3 and ref. [112] for a more detailed discussion of this point.
} 
3. Asymmetric Fermi function (two parameters):

$$
\rho(z)=\frac{\rho_{\infty}}{\left[1+\exp \left(z / \alpha_{3}\right)\right]^{\gamma_{3}}} .
$$

4. A three-parameter function which has the correct mathematical asymptotic behaviour both for $z \rightarrow-\infty$ and $z \rightarrow+\infty$ (see section 3.3):

$$
\rho(z)=\rho_{\infty}\left[A(z) \frac{1}{1+\exp \left(z / \alpha_{4}\right)}+(1-A(z)) \frac{c}{z^{6}+\beta_{4}}\right]
$$

Hereby $A(z)$ is given by

$$
A(z)=\frac{1}{1+\exp \left(z / \gamma_{4}\right)}
$$

and the constant $c$ by eq. (3.31).

In table 7 we list the solutions of the variational calculation; for each form of $\rho(z)$ the minimizing parameters and the corresponding surface energy are given for four of the Skyrme forces listed in table 1. Of the two one-parameter profiles, the Fermi function is clearly favoured as compared to the error function which has recently been discussed in the analysis of nuclear density profiles [88]. Introducing an asymmetry of the surface around the inflection point by the profile eq. (5.36) lowers the surface energy by about $0.2-0.3 \mathrm{MeV}$; the effect is smallest for the force SIII which has the largest incompressibility $K_{\infty}$. It is rather interesting to note now that the introduction of one more variational parameter in eq. (5.37) hardly lowers the surface energy any more. In particular, the seemingly unphysical asymptotic behaviour $\rho(z) \sim z^{-6}$ is shown hereby to play no role for the quantity of physical interest, namely the surface energy $a_{\mathrm{s}}^{\infty}$. This strongly confirms our conclusions drawn at the end of section 3.3 and encourages us to use the ETF functionals including the 4th order terms for variational calculations with trial functions. We learn furthermore from table 7 that the asymmetric Fermi functions eq. (5.36) minimize the surface energy to within $0.02-0.03 \mathrm{MeV}$ for forces with realistic incompressibility (i.e. SkM and SkM*) as compared to the profiles with one more parameter; this corresponds to about $1 \mathrm{MeV}$ in a heavy nucleus. This gives a confirmation of the density profiles we have used in the calculations of finite nuclei, see eq. (4.1).

Table 7

\begin{tabular}{|c|c|c|c|c|c|c|c|c|c|c|c|}
\hline \multirow[b]{2}{*}{ Force } & \multicolumn{2}{|c|}{ eq. (5.34) } & \multicolumn{2}{|c|}{ eq. (5.35) } & \multicolumn{3}{|c|}{ eq. (5.36) } & \multicolumn{4}{|c|}{ eq. (5.37) } \\
\hline & $\alpha_{1}$ & $a_{s}^{\infty}$ & $\alpha_{2}$ & $a_{3}^{\infty}$ & $\alpha_{3}$ & $\gamma_{3}$ & $a_{z}^{\infty}$ & $\alpha_{4}$ & $\beta_{4}$ & $\gamma_{4}$ & $a_{s}^{\infty}$ \\
\hline SIII & 0.425 & 18.13 & 1.14 & 18.69 & 0.485 & 1.34 & 18.04 & 0.553 & 70.1 & 0.553 & 18.09 \\
\hline Ska & 0.484 & 18.79 & 1.27 & 18.99 & 0.602 & 1.70 & 18.52 & 0.746 & 69.8 & 0.530 & 18.49 \\
\hline SkM & 0.486 & 16.85 & 1.28 & 17.03 & 0.605 & 1.69 & 16.60 & 0.731 & 69.8 & 0.548 & 16.58 \\
\hline SkM* & 0.501 & 17.51 & 1.31 & 17.64 & 0.634 & 1.78 & 17.22 & 0.795 & 69.8 & 0.534 & 17.19 \\
\hline
\end{tabular}

Minimal surface energies $a_{k}$ (in $\mathrm{MeV}$ ) and optimal variational parameters obtained for semi-infinite nuclear matter with different parametrisations (see text) of the density profile, using various forces 
Another measure for the convergence of this search of profiles is given in the following. The surface tension $\sigma$ defined by

$$
a_{\mathrm{s}}^{\infty}=4 \pi r_{0}^{2} \sigma
$$

can be shown to be stationary (at $\rho_{0}=\rho_{\infty}$ )

$$
\dot{\sigma}=\left.\rho_{\infty} \frac{\mathrm{d} \sigma}{\mathrm{d} \rho_{0}}\right|_{\rho_{\infty}}=0,
$$

if the exact solution $\rho(z)$ of the Euler equation has been used (see refs. $[19,89])$. The value of the quantity $\dot{\sigma}$ found in numerical calculations is thus a measure for the quality of the profile $\rho(z)$ used (or found). For the force SkM* we found for

Fermi function eq. (5.34): $\quad \dot{\sigma} / \sigma=0.21$,

asymm. Fermi fct. eq. (5.36): $\quad \dot{\sigma} / \sigma=0.02$;

thus clearly indicating the improvement gained by introducing the asymmetry of the surface profile. The relative inaccuracy of $2 \%$ of the violation of the theorem eq. (5.40) is found to have no practical influence on the calculation of the droplet model parameters and their relations discussed in the next section. A consequence of eqs. (5.39) and (5.40) is that

$$
\dot{a}_{\mathrm{s}}=-\frac{2}{3} a_{\mathrm{s}}^{\infty},
$$

which we have already used in eq. (5.29).

Encouraged by these results, we can go ahead using the asymmetric Fermi type profiles eq. (5.36) and calculate the asymptotic parameters of the leptodermous expansion of the energy discussed in the last section. As eqs. (5.9), $(5.20,5.21)$ tell us, the curvature energy $a_{c}^{\infty}$ and the next coefficient $a_{0}^{\infty}$, as well as their derivatives defined as in eq. (5.26), can be obtained from the semi-infinite density profile $\rho(z)$ in the limit $\rho_{0}=\rho_{\infty}$. In table 8 we list these quantities for the various Skyrme forces and also for the Tondeur energy density discussed earlier. Their significance for LDM type or droplet model mass formulae shall be discussed in the section 5.3 below.

Some of the surface energy coefficients listed in table 8 have also been calculated in the Hartree-

Table 8

Symmetric LDM parameters (in MeV) obtained for various forces with parametrisation (5.36) of the semi-infinite density profile. (For $\dot{\sigma} / \sigma$, see eq. (5.40) and text thereafter.)

\begin{tabular}{lllllllllll}
\hline Force & \multicolumn{1}{c}{$a_{\mathrm{s}}^{\infty}$} & \multicolumn{1}{c}{$\dot{a}_{\mathrm{s}}$} & \multicolumn{1}{c}{$\ddot{a}_{\mathrm{s}}$} & \multicolumn{1}{c}{$a_{\mathrm{c}}^{\infty}$} & \multicolumn{1}{c}{$\dot{a}_{\mathrm{c}}$} & \multicolumn{1}{c}{$\vec{a}_{\mathrm{c}}$} & \multicolumn{1}{c}{$a_{\mathbf{0}}^{\infty}$} & $a_{\mathrm{c}}^{*}$ & $a_{0}^{*}$ & $\dot{\sigma} / \sigma$ \\
\hline SIII & 18.04 & -12.40 & -88.11 & 9.52 & 26.02 & 49.32 & -8.66 & 7.57 & -4.8 & -0.02 \\
Ska & 18.52 & -11.60 & -71.29 & 12.15 & 30.20 & 47.28 & -13.88 & 9.86 & -7.6 & 0.04 \\
SkM & 16.60 & -11.06 & -58.64 & 12.19 & 27.26 & 37.27 & -12.31 & 9.65 & -6.2 & 0.003 \\
SkM & 17.22 & -11.15 & -60.37 & 12.82 & 29.27 & 40.76 & -14.13 & 10.24 & -7.3 & 0.02 \\
Tond. & 18.11 & -11.30 & -65.19 & 12.74 & 30.25 & 44.23 & -14.73 & 10.32 & -7.9 & 0.04 \\
RATP & 18.48 & -11.38 & -70.11 & 12.99 & 30.36 & 42.05 & -15.70 & 10.56 & -8.8 & 0.06 \\
\hline
\end{tabular}


Fock approximation [90]. The HF results are typically $\sim 0.5-0.8 \mathrm{MeV}$ higher than our semiclassical results. (For a more detailed comparison, see ref. [72].) A part of this discrepancy is related to the systematic overbinding found in the ETF model, as discussed in section 4.2 above. This explains, however, only about one half of the difference between the HF and the ETF results for $a_{s}^{\infty}$. It should be noted that the numerical uncertainties in the semi-infinite HF results, which are mainly due to the so-called Friedel oscillations, easily amount to $\sim 0.5 \mathrm{MeV}$ or more [90]. It also is not clear whether these oscillations - which by construction are absent in the ETF calculations - do not contribute to $a_{s}^{\infty}$. They are known to contribute immensely to the curvature energy coefficient $a_{c}^{\infty}$ and make it, in fact, impossible to extract unique values of $a_{\mathrm{s}}^{\infty}$ from the semi-infinite HF density profiles [91].

Before going to the asymmetric case, we want to mention that for a number of simplified energy density functionals using only second order corrections to the kinetic energy density functional $\tau[\rho]$, it is possible to solve analytically the Euler equation for the semi-infinite case and interesting relations between the basic constants and the principal LDM parameters can be derived $[61,89,92,121]$.

\subsubsection{Asymmetric case}

We shall now consider semi-infinite nuclear matter, treating the neutrons and protons separately (still leaving out the Coulomb interaction). In the saturated limit $z \rightarrow-\infty$ their densities shall be $\rho_{0 p}$ and $\rho_{0 n}$. As a measure for the asymmetry we introduce the quantity $\delta$ of the droplet model [19]:

$$
\delta=\left(\rho_{0 \mathrm{n}}-\rho_{0 \mathrm{p}}\right) / \rho_{0} ; \quad \rho_{0}=\rho_{0 \mathrm{n}}+\rho_{0 \mathrm{p}}
$$

The profiles $\rho_{\mathrm{q}}(z)$ are chosen to be asymmetric Fermi functions, based on our results of the symmetric semi-infinite case:

$$
\rho_{\mathrm{q}}(z)=\rho_{0 \mathrm{q}} /\left[1+\exp \left(\frac{z-Z_{\mathrm{q}}}{\alpha_{\mathrm{q}}}\right)\right]^{\gamma_{\mathrm{q}}} .
$$

Since the relative position of the two surfaces along the $z$-axis is free, we have introduced the variables $Z_{\mathbf{q}}$. However, because of the translational invariance of the variational equation, only their difference is of interest; we define thus the "neutron skin parameter" $\tau$ by

$$
\tau=\frac{1}{r_{0}}\left(Z_{\mathrm{n}}-Z_{\mathrm{p}}\right) .
$$

The variational calculation is thus in principle done by minimizing the surface energy eq. (5.32) - with $\rho(z)=\rho_{\mathrm{n}}(z)+\rho_{\mathrm{p}}(z)$ - with respect to the five parameters $\alpha_{\mathrm{q}}, \gamma_{\mathrm{q}}$ and $\tau$ for a fixed value of the asymmetry parameter $\delta$.

Our main interest here is to determine the parameters of the droplet model [19]. We expand thus the surface energy as in ref. [19]:

$$
a_{\mathrm{s}}=a_{\mathrm{s}}^{\infty}+H \tau^{2}+2 P \tau \delta-G \delta^{2} .
$$

The corresponding expansion of the volume energy $a_{v}$ (eq. (5.33)) is

$$
a_{\mathrm{v}}=a_{\mathrm{v}}^{\infty}+\frac{1}{2} K_{\infty} \varepsilon^{2}+J \delta^{2}-L \varepsilon \delta^{2}+\frac{1}{2} M \delta^{4},
$$


where $\varepsilon$ is defined in eq. (5.22). Here we have added also a 4th order term in $\delta$, as it was introduced in ref. [19]. It turns out, however, that the coefficient $M$ is so small for all realistic effective forces (see table 2), that the $\delta^{4}$ term in eq. (5.46) and the corresponding contributions to the surface energy, eq. (5.45), can be safely neglected in applications to finite nuclei, where $\delta$ is rarely larger than $\sim 0.2$.

From the saturation condition $\mathrm{d} a_{\mathrm{v}} / \mathrm{d} \varepsilon=0$, one finds then from eq. (5.46)

$$
\varepsilon=\frac{L}{K_{\infty}} \delta^{2}
$$

This value has been imposed (thus determining $\rho_{0}$ ) in all the calculations discussed in the following. (Therefore no terms containing $\varepsilon$ appear in eq. (5.45).) Fixing selected values of $\tau$ and $\delta$ and varying the remaining parameters of the density profiles, the constants $H, P$ and $G$ are easily found.

Since the semi-infinite system under discussion here is to be thought of as the limiting case of the surface profile of a very heavy nucleus, the quantities $\tau$ and $\delta$ are, in fact, not independent. Using the same profiles as in eq. (5.43) for spherical nuclei, replacing $z$ by the radial variable $r$ and $Z_{\mathrm{q}}$ by the radii $R_{\mathrm{q}}, \tau$ in eq. (5.44) becomes the "neutron skin" parameter of a finite nucleus

$$
\tau=t / r_{0}=\left(R_{\mathrm{n}}-R_{\mathrm{p}}\right) / r_{0}
$$

It is now a simple matter to use the particle conservation in the leptodermous approximation eq. (5.14) - but separately for $N$ neutrons and $Z$ protons - and to show that

$$
\tau=\frac{2}{3}(I-\delta) A^{1 / 3}-\left(c_{0}^{\mathrm{n}} \alpha_{\mathrm{n}} / r_{0}-c_{0}^{\mathrm{p}} \alpha_{\mathrm{p}} / r_{0}\right)+\mathcal{O}\left(A^{-1 / 3}\right),
$$

where the $c^{g}$ are defined in eq. (C.20) of appendix C and

$$
I=(N-Z) / A
$$

The second term, of order $A^{0}$ in eq. (5.49), comes from the asymmetry of the surface profiles $\left(\gamma_{q} \neq 1\right)$; it is missing in the droplet model [19]. This term is not negligible in realistic cases $\left(c_{0}^{\mathrm{g}} \simeq-0.6\right.$ to -0.9$)$. However, in order to make contact with the droplet model [19] and its relations, we have restricted our following analysis to the case of pure Fermi function profiles $\left(\gamma_{q}=1\right)$, when the $c_{0}^{q}$ are equal to zero.

\begin{tabular}{|c|c|c|c|c|c|c|c|}
\hline Force & $a_{s}^{\infty}$ & $H$ & $P$ & $G$ & $J$ & $Q$ & $\tilde{J}$ \\
\hline$M \& S^{*}$ & 18.56 & 9.42 & 17.55 & 45.4 & 28.06 & 16.1 & 27.94 \\
\hline SIII & 18.13 & 13.55 & 31.76 & 26.52 & 28.16 & 54.6 & 28.72 \\
\hline Ska & 18.79 & 10.66 & 31.69 & 47.47 & 32.91 & 29.8 & 31.77 \\
\hline SkM & 16.85 & 11.11 & 31.99 & 38.94 & 30.75 & 36.3 & 30.34 \\
\hline SkM* & 17.51 & 10.56 & 31.61 & 39.04 & 30.03 & 35.4 & 29.77 \\
\hline Tondeur & 18.41 & 11.46 & 33.24 & 39.83 & 32.12 & 37.0 & 31.32 \\
\hline RATP & 18.80 & 11.73 & 32.58 & 33.05 & 29.26 & 45.6 & 29.65 \\
\hline
\end{tabular}

Table 9

Surface energy and volume asymmetry energy parameters (in MeV) obtained for various forces

${ }^{*}$ See ref. [19]. 
Also, keeping the second term in eq. (5.49) and consistently all other lowest order contributions of the diffuseness parameters $\alpha_{\mathrm{q}}$ in the relations to be discussed below would complicate the analysis appreciably. We believe that this restriction is not serious, having seen that the total binding energy of real heavy nuclei (including Coulomb) is lowered only by about $5 \mathrm{MeV}$ when the parameters $\gamma_{q}$ are varied away from $\gamma_{\mathrm{q}}=1$ (see section 4.2).

In table 9 we have listed the constants $H, P$ and $G$ of eq. (5.45) for various forces, including also the values of Myers and Swiatecki [19]. Also shown in the table are the volume asymmetry $J$ (eq. (2.18)), the so-called "surface stiffness coefficient" $Q$ of the droplet model defined by

$$
Q=H /\left(1-\frac{2}{3} P / J\right)
$$

as well as the quantity $\tilde{J}$

$$
\tilde{J}=\frac{2}{3}(P+G H / P)
$$

of which a theorem proved in ref. [19] says that it is equal to $J$. We see from table 9 that, indeed, this theorem is fulfilled in all cases within less than $3 \%$. Our values of $Q$ agree well (within $\$ 10 \%$ ) with those reported from semi-infinite HF calculations [90], considering the rather large numerical uncertainties of the latter.

\subsection{Discussion of the droplet model}

Our semiclassical variational calculations presented above give an ideal starting point for a discussion of the droplet model (DM) by Myers and Swiatecki [19]. Ideal in the sense that the use of parametrized density profiles allows to directly perform the leptodermous expansion of the energy shown in section 5.1 above. Myers and Swiatecki performed density variational calculations with the Seyler-Blanchard force [93] to determine some of the DM parameters. Our calculations represent a "modernization" of this procedure in two respects: first, we have now effective forces at hand which reproduce the binding energies and many other bulk properties of nuclei very. well and second, we have included the semiclassical corrections to the functional $\tau[\rho]$ which we have shown in section 3.2 to be important to obtain the correct kinetic energies (in ref. [19] only the TF relation $\tau_{\mathrm{TF}}[\rho]$ was used). For a series of effective forces, we have given the infinite nuclear matter properties in table 2 . The coefficients appearing in the leptodermous expansion of the energy of symmetric nuclei have been given in table 8 and the surface asymmetry coefficients in table 9 . We have thus all the ingredients ready to discuss some of the DM relations and to compare the DM parameters to those found above.

\subsubsection{Curvature energy}

The curvature energy coefficient $a_{c}^{\infty}$ is found to be of the order of $\sim 9-12 \mathrm{MeV}$ for all forces. This is in agreement with the result found in the original TF calculations in ref. [19]. However, in all succeeding DM applications and in most mass table calculations using the DM or similar relations (see refs. $[94,95])$, the coefficient $a_{c}^{\infty}$ has been put equal to zero.

It should be said that $a_{c}^{\infty}$ (or $a_{c}^{*}$ in eq. (5.30)) is not easily determined from fits to experimental nuclear masses. In any mass fit to a truncated expansion of the type of eq. (5.30)-including asymmetry and Coulomb terms - the coefficients found by the fit will depend on the number of terms of the expansion included and on the precise analytical form chosen for the dependence of the masses on the 
nucleon numbers; of course, they will also depend on the number of experimental data included. In ref. [86] we showed with a schematic Skyrme force that the curvature energy $a_{c}^{*}$ easily can vary by $2-4 \mathrm{MeV}$, depending on these conditions; the situation becomes even worse for the lower order terms $a_{0}^{*}$ etc. Therefore, it does not make sense to compare the values of the different parameters obtained in various mass fits. The only thing which can be discussed are the asymptotic values, $a_{\mathrm{s}}^{\infty}, a_{\mathrm{c}}^{\infty}$ etc. found in semi-infinite nuclear matter calculations.

Still, we believe that there is no reason to ignore the curvature energy term $a_{\mathrm{c}}^{*} A^{1 / 3}$ in the energy, since all realistic forces give $a_{c}^{*}$ of the order of $10 \mathrm{MeV}$ (the compression energy in eq. (5.31) is typically only $\sim-2$ to $-3 \mathrm{MeV}$ ), which corresponds to some $50-70 \mathrm{MeV}$ in the total energy of heavy nuclei.

\subsubsection{Compression effects}

The quantity $\varepsilon$ (eq. (5.22)) measures the compression of the nucleus by the surface tension; it would be zero if the nuclear matter were totally incompressible $\left(K_{\infty}=\infty\right)$, as can be seen from eq. (5.27). That expression was derived above only for the symmetric case without Coulomb interaction. The latter tends to counterbalance the surface effect and becomes dominating for heavy nuclei. If we add the lowest order Coulomb and asymmetry contributions to $\varepsilon$ (compare eq. (5.47) for the latter) as in ref. [19], eq. (5.27) would be modified to

$$
\varepsilon(A)=\frac{3 \dot{a}_{\mathrm{s}} A^{-1 / 3}+3 \dot{a}_{\mathrm{c}} A^{-2 / 3} \pm L \delta^{2}+c_{1} Z^{2} A^{-4 / 3}}{K_{\infty}+9 \ddot{a}_{\mathrm{s}} A^{-1 / 3}+9 \ddot{a}_{\mathrm{c}} A^{-2 / 3}}
$$

where $c_{1}$ is the usual Coulomb energy coefficient

$$
c_{1}=\frac{3}{5} e^{2} / r_{0}
$$

In the DM the terms containing $\dot{a}_{\mathrm{c}}, \ddot{a}_{\mathrm{s}}$ and $\ddot{a}_{\mathrm{c}}$ are missing; as we have seen in section 5.1 this leads to large errors if $K_{\infty}$ is of the order of $\sim 220 \mathrm{MeV}$. To our opinion, this is a serious drawback of the DM in its present form. The most important term missing there is the surface compressibility $\ddot{a}_{\mathrm{s}}$ which is known to play an important role in determining the compressibility of finite nuclei $[29,31,89]$. If we define a "bulk compressibility" $K_{\mathrm{A}}^{\text {bulk }}$ of a finite nucleus by

$$
K_{\mathrm{A}}^{\text {bulk }}=9 \rho_{0}^{2} \frac{\mathrm{d}^{2}}{\mathrm{~d} \rho_{0}^{2}}\left(\frac{E}{A}\right),
$$

keeping the surface diffusivity $\alpha$ constant, - other compressibilities taking changes of $\alpha$ into account play a role, too $[89,96]$ - we find that the leading terms in $K_{\mathrm{A}}^{\text {bulk }}$ are

$$
K_{\mathrm{A}}^{\text {bulk }}=\left(\rho_{0} / \rho_{\infty}\right)^{2}\left(K_{\infty}+9 \ddot{a}_{\mathrm{s}} A^{-1 / 3}+9 \ddot{a}_{\mathrm{c}} A^{-2 / 3}\right) .
$$

We can see, using the values of $\ddot{a}_{\mathrm{s}}$ and $\ddot{a}_{\mathrm{c}}$ in table 8 , that their contribution even in heavy nuclei is by no means negligible, in particular that of $\ddot{a}_{\mathrm{s}}$. (The terms $\ddot{a}_{\mathrm{v}}$ in eq. (5.23) also gives a noticeable contribution to $K_{A}^{\text {bulk }}[89]$.) Note that the term in brackets in eq. (5.55) is identical to the denominator of $\varepsilon(A)$ (eq. (5.52)). The possibility of extending the DM definition of $\varepsilon$ was recently questioned [97], pointing out that in expressions like eq. (5.52) a pole might arise for some (small) value of $A$ (noting that $\ddot{a}_{\mathrm{s}}$ is negative). However, this conclusion was premature; it was due to the omission of $\ddot{a}_{\mathrm{c}}$ in the denominator. 
In fact, by looking at eq. (5.55) we can see that the denominator of eq. (5.52) will always be positive since for physical reasons, the compressibility $K_{A}^{\text {bulk }}$ certainly is positive.

In eq. (5.52) we have still not taken into account the asymmetry and Coulomb contributions to the compressibility; they are known to contribute noticeably to $K_{A}^{\text {bulk }}[31,96]$ in heavy nuclei and would appear in the denominator of $\varepsilon(A)$, too. This will ultimately render the expression for $\varepsilon(A)$ rather complicated and, consequently, the extended DM relations will be very cumbersome.

\subsubsection{Surface stiffness parameter}

The parameter $Q$ (eq. (5.51)) appears in the DM relation between the "microscopic" and the "macroscopic" asymmetry parameters $\delta$ and $I$, respectively:

$$
\delta=I /\left(1+\frac{9}{4} \frac{J}{Q} A^{-1 / 3}\right)
$$

(without Coulomb contribution to be discussed in paragraph 5.3.4 below). We have tested eq. (5.56) by calculating a series of finite nuclei (without Coulomb interaction) with a fixed value of $I$. Reading off the numerical values of $\delta$ (eq. (5.42)) from the variational densities, we solved eq. (5.56) for $Q$ and plotted it versus $A^{-1 / 3}$. The result is shown in fig. 12 for the forces SIII and SkM*. We see that for large values of $A$, the value of $Q$ (the crosses) form a straight line which can be rather safely extrapolated to $A^{-1 / 3}=0$. The asymptotic values found for the surface stiffness parameter $Q$ in this way agree exactly with the values found in the semi-infinite matter calculations reported in section 5.2 above (see table 9). This provides thus a nice test of the consistency of both kinds of calculations.

The coefficient $Q$ determines what is often called the "surface asymmetry energy" which we shall denote here by $k_{\mathrm{s}}$. It is defined in extending the usual LDM expression for the binding energy in the

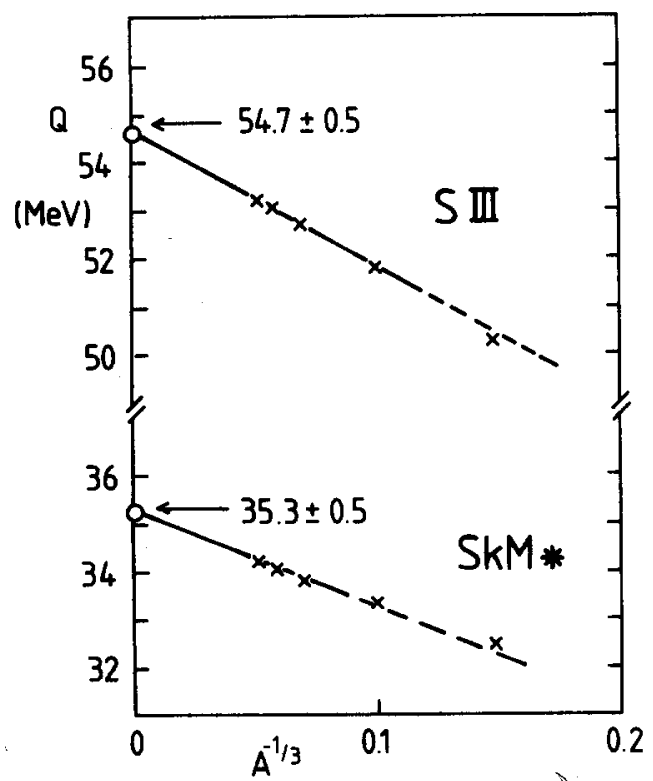

Fig. 12. Surface stiffness parameter $Q$ versus $A^{-1 / 3}$, obtained from variational ETF caleulations for asymmetric finite nuclei (no Coulomb), using the forces SIII and SkM ${ }^{*}$. The extrapolated values at $\boldsymbol{A}^{-1 / 3}=0$ are to be compared with those obtained in semi-infinite nuclear matter calculations given in table 9. 
following way:

$$
E(A, I)=\left(a_{\mathrm{v}}^{\infty}+J I^{2}\right) A+\left(a_{\mathrm{s}}^{\infty}+k_{\mathrm{s}} I^{2}\right) A^{2 / 3}+\cdots .
$$

In the DM the expression for the total asymmetry energy is

$$
E_{\mathrm{as}}=J A I \delta=J A I^{2} /\left(1+\frac{9}{4} \frac{J}{Q} A^{-1 / 3}\right) \quad\left[+\mathcal{O}\left(I^{4}\right)\right]
$$

which we have verified numerically to be valid within $<10^{-3}$ for $I \leqslant 0.2$. As emphasized by Myers and Swiatecki [19], the expression (5.58) may not be Taylor-expanded in powers of $A^{-1 / 3}$, since the second term in the denominator is all but small for real nuclei $\left(\frac{9}{4} \mathrm{~J} / Q=1-3\right.$ depending on the force, see table 9 , and $A^{-1 / 3} \sim \frac{1}{6}$ for heavy nuclei). Expanding nevertheless, one gets

$$
E_{\mathrm{as}} \approx I^{2}\left(J A-\frac{9}{4} \frac{J^{2}}{Q} A^{2 / 3}+\cdots\right)
$$

implying that

$$
k_{\mathrm{s}}=-\frac{9}{4} J^{2} / Q
$$

(A small contribution $2 a_{s}^{\infty} L / K_{\infty}$ to $k_{\mathrm{s}}$, given in ref. [19], is contained in our definition eq. (5.60) due to the way in which we have calculated the values of $Q$ given in table 9 by including the contribution of eq. (5.47) into the coefficient $G$ of eq. (5.45).)

We note again that the expansion eq. (5.59) is only legitimate asymptotically, i.e. for very large values of $A$. In mass fits to finite nuclei, the form (5.58) must be kept. The convergence of eq. (5.59) depends on the value of $Q$ which is not well known. A look at table 9 reveals that $Q$ varies appreciably between different forces. Still, it seems that all realistic forces give larger values than that Myers and Swiatecki [19] by a factor of 2-3. Their low value of $Q$ tends to overestimate the "neutron skin thickness"; this has been observed in systematic comparisons with HF results [32,98].

The variation of $Q$ seen in table 9 reflects the fact that this parameter is not easy to determine in mass fits. A recent analysis [72], taking into account fission barrier heights (which are very sensitive to the surface asymmetry energy) and the available data on the "neutron skin thickness" $t$ (see section 4.2), leads to the conclusion that, indeed, values of $Q \geq 40$ seem to be indicated. The surface asymmetry properties of the present-day effective forces, reflected in $Q$, are still a weak point. They play a crucial role in astrophysics where one e.g. needs extrapolations to isotopes far away from the $\beta$-stability line in $r$-process calculations.*

\subsubsection{Coulomb energy contributions}

In the leptodermous expansion discussed in section 5.1 we have left out the Coulomb energy. Its expansion will be discussed in appendix D. In the DM relations, it is sufficient to take into account only the lowest order Coulomb contributions corresponding to a sharp LDM surface. The DM expression for

* Note that the Skyrme force RATP, which was adjusted especially to the properties of neutron matter [120], also predicts a value $Q \approx 45 \mathrm{MeV}$. 
$\delta$ then becomes [19]:

$$
\delta=\left(I+\frac{3}{8} \frac{c_{1}}{Q} Z^{2} A^{-5 / 3}\right) /\left(1+\frac{9}{4} \frac{J}{Q} A^{-1 / 3}\right)
$$

We have tested this relation numerically from the variational results of realistic finite nuclei. We found it not to work well. Taking the Coulomb-free part of eq. (5.61) for granted with the parameters checked above, we found that the Coulomb contribution to $\delta$ is underestimated in the DM relation eq. (5.61) by a factor of $\sim 2$ in light nuclei and up to $\sim 4$ in heavy nuclei. (Similarly, we found the so-called Coulomb redistribution coefficient $c_{2}$ to be larger by factors $\sim 2-3$ than the DM value.)

We have not been able to trace down the origin of this discrepancy. In order to come up with a more satisfactory relation for $\delta$, we rederive it in the following in a very simple way.

Let us solve the variational equation (3.22) locally at the centre of a heavy nucleus. There, we may neglect the contributions from all the gradient terms since the density is flat. We thus can replace $\mathscr{E}\left[\rho_{\mathrm{n}}, \rho_{\mathrm{p}}\right]$ by

$$
\mathscr{E}\left[\rho_{0 \mathrm{n}}, \rho_{\mathrm{op}}\right]=\rho_{0} a_{\mathrm{v}}+\frac{1}{2} V_{\mathrm{c}}(0) \rho_{0 \mathrm{p}}
$$

where $a_{v}$ is given by eq. (5.46) and $V_{c}(0)$ is the Coulomb potential at the origin, which for a sharp-surface proton distribution is

$$
V_{\mathrm{c}}(0)=\frac{3}{2} Z e^{2} / R_{\mathrm{p}} \simeq \frac{5}{2} c_{1} Z A^{-1 / 3}(1-\varepsilon) .
$$

Doing the variation of the $\rho_{0 \mathrm{q}}$ at the origin, we get an expression for the Fermi energies $\lambda_{\mathrm{q}}$ as functions of $\varepsilon$ and $\delta$ :

$$
\lambda_{\mathrm{q}}=\partial \mathscr{E} / \partial \rho_{0 \mathrm{q}}=a_{\mathrm{v}}^{\infty}-\frac{1}{3} K_{\infty} \varepsilon-J \delta^{2}+\frac{1}{3} L \delta^{2} \pm 2 \delta J \mp 2 L \varepsilon \delta+V_{\mathrm{c}}(0) \delta_{\mathrm{q}, \mathrm{p}},
$$

where the upper signs are for $q=n$ and the lower for $q=p$. The Fermi energies can also be found from the total energy which we write, based on the above results, as

$$
E(A, I)=a_{\mathrm{v}}^{\infty} A+a_{\mathrm{s}}^{\infty} A^{2 / 3}+a_{\mathrm{c}}^{*} A^{1 / 3}+c_{1} Z^{2} A^{-1 / 3}+A J I^{2} /\left(1+\frac{9}{4} \frac{J}{Q} A^{-1 / 3}\right) .
$$

Using the definitions $\partial E / \partial N=\lambda_{n}, \partial E / \partial Z=\lambda_{p}$, we find from eq. (5.65)

$$
\lambda_{\mathrm{q}}=a_{\mathrm{v}}^{\infty}+\frac{2}{3} a_{\mathrm{s}}^{\infty} A^{-1 / 3}+\frac{1}{3} a_{\mathrm{c}}^{*} A^{-2 / 3}-\frac{1}{3} c_{1} Z^{2} A^{-4 / 3}+2 c_{1} Z A^{-1 / 3} \delta_{\mathrm{q}, \mathrm{p}}+\left( \pm 2 I J-J I^{2}\right) /\left(1+\frac{9}{4} \frac{J}{Q} A^{-1 / 3}\right) .
$$

Equating the expressions (5.64) and (5.66) for $\lambda_{q}$, we obtain two new equations. Taking their difference, we find

$$
\delta=I /\left(1+\frac{9}{4} \frac{J}{Q} A^{-1 / 3}\right)+\frac{c_{1}}{8 J} Z A^{-1 / 3}
$$


Hereby the symmetric part of the neglected surface terms in the variational equation have cancelled, so that we are sure to have all leading terms in $\delta$ included in (5.67). (Had we taken the sum of the above equations, we would have obtained the leading terms of $\varepsilon(A)$; there, however, the surface terms such as $\dot{a}_{c}, \ddot{a}_{s}$, etc. would have been important as discussed above.)

Equation (5.67) was found in our numerical calculations to reproduce the Coulomb part of $\delta$ within $\leqslant 10 \%$, thus bringing a considerable improvement over the DM relation (5.61). Note that apart from terms of order $A^{-2 / 3}$, the main differences are the appearance of $J$ in our relation compared to $Q / 3$ in the DM relation, and the different power-law dependences on $Z$ and $A$ of the Coulomb term.

\subsubsection{Conclusions}

In conclusion we can say that our calculations confirm the structure of the DM relations, as must be expected. However, we have seen that at some places the expansions made in the DM have not been carried far enough to give a quantitative agreement with numerical results. We further note the absence in the DM of all the curvature contributions containing $a_{c}^{\infty}, \dot{a}_{c}$ and $\ddot{a}_{\mathrm{c}}$ which we have seen to be non-negligible; there also is a discrepancy with the Coulomb contributions.

In two cases we have shown ways of improving or extending the DM relations. To do this consistently including simultaneously all effects of compressibility, asymmetry and Coulomb would, however, be very tedious; even more so if we would take the surface diffuseness parameters $\alpha_{q}$ (and $\gamma_{q}$ ) explicitly into account which, as we have seen in section 4.2 , seem indispensable to obtain a flexible enough description of the "neutron skin".

There is also a limit to the usefulness of such extensions. From the above results, it can easily be estimated that some 3-6 (or more) new phenomenological parameters (such as $\ddot{a}_{s}, \dot{a}_{\mathrm{c}}, \ddot{a}_{\mathrm{c}}$ and their analogs for the asymmetric part of the energy) would have to be introduced in order to describe quantitatively the compression effects obtained with realistic forces. This would easily lead to a lot of 20 or more phenomenological DM parameters. More than half of them are redundant in the sense that the whole model can be derived by ETF calculations using a Skyrme force with less than 10 parameters. Their interrelations are implicitly given by the force, but not explicitly known (unless some more theorems are derived or simple soluble models are used such as in refs. [61, 89, 92]).

We therefore would advocate, instead of performing all the expansions and approximations necessary to extend and improve the droplet model, to use the numerical results of the variational ETF calculations which, as we believe to have demonstrated, are easily and efficiently obtained and reproduce quantitatively the average results of $\mathrm{HF}$ calculations using the same effective interactions.

\section{Extension to nuclear systems at finite temperature}

In this section we shall discuss extensions of semiclassical methods to nuclear systems at finite temperature. We have already in the introduction mentioned that the newest developments in heavy ion and astrophysics strongly motivate the investigation of highly excited nuclear systems. These are conveniently described in the statistical approach in terms of a finite temperature $T$. In the mean field (HF) theory one then minimizes no longer the total intrinsic energy $E$ but the Helmholtz free energy $F$ (at constant temperature)

$$
F=E-T S
$$


The entropy $S$ is given by

$$
S=-\sum_{\mathfrak{q} \nu}\left[n_{\nu}^{\mathfrak{q}} \ln n_{\nu}^{\mathfrak{q}}+\left(1-n_{\nu}^{\mathfrak{q}}\right) \ln \left(1-n_{\nu}^{\mathfrak{q}}\right)\right]
$$

in terms of the Fermi occupations numbers

$$
n_{\nu}^{\mathfrak{q}}=1 /\left\{1+\exp \left(\frac{\varepsilon_{\nu}^{\mathfrak{q}}-\lambda_{\mathbf{q}}}{T}\right)\right\}
$$

(We put the Boltzmann constant $k$ equal to unity and measure the temperature $T$ in units of $\mathrm{MeV}$.)

HF calculations at finite temperatures are relatively easy to perform; it is sufficient to replace the HF occupation numbers $n_{\nu}^{q}$ in eqs. (2.2)-(2.4) by the finite-T occupation numbers (eq. (6.3)). Such calculations were performed with Skyrme forces by different groups $[23,99,100]$. Hereby it must be assumed that the parameters of the Skyrme force themselves do not depend on $T$. This could in principle be checked by performing a Brückner $G$-matrix calculation at finite temperature; such a calculation has, however, not been endeavoured so far.

A well-known effect of the smoothing of the Fermi surface brought about by the occupation numbers eq. (6.3) is the washing-out of the shell effects; the above-mentioned HF results showed that beyond a critical temperature $T_{\mathrm{c}} \simeq(2.5-3) \mathrm{MeV}$ (which is roughly the same for all systems), the shell effects have disappeared. Systems at such temperatures are thus ideal objects for studies within a semiclassical framework. It is therefore obvious to try to apply the methods developed above to nuclei at $T>0$.

\subsection{Earlier approaches}

Most semiclassical calculations at $T>0$ performed until now were done in the TF approximation [101-103]. Some authors - well aware that gradient corrections are important for finite nuclei-used either of the following approximative ways to include gradient corrections to the kinetic energy density functional $\tau[\rho]$.

\subsection{1. "Hot" TF functional with "cold" gradient corrections}

One way is to add to the exact TF functional $\tau_{\text {TF }}^{T}[\rho]$, valid for $T>0$ (and given in eq. (6.29) below), the gradient corrections $\tau_{2}[\rho]$ (and $\tau_{4}[\rho]$ ) known from the $T=0$ case in an ad hoc manner:

$$
\tau_{\mathrm{ETF}}^{T}[\rho]=\tau_{\mathrm{TF}}^{T}[\rho]+\tau_{2}^{T=0}[\rho]\left(+\tau_{4}^{T=0}[\rho]\right)
$$

Along with eq. (6.4), the pure TF functional for the entropy density (see below) is used, since no gradient corrections to it can be guessed from the $T=0$ case.

This procedure, proposed originally by Barranco and Treiner [104] (but without $\tau_{4}[\rho]$ and with a readjusted Weizsäcker coefficient), is inconsistent in two ways. First, it neglects gradient (i.e. finite size) corrections to the entropy density. Second, it neglects the temperature dependence of the gradient corrections to $\tau[\rho]$, which we shall discuss below. Nevertheless, it leads to reasonable results for spherical systems [105]. However, this approximation failed to yield reasonable average deformation energies at $T>0$, which can be traced back to the absence of gradient corrections to the entropy density. 


\subsubsection{Low-temperature expansion}

If one expands the TF term in eq. (6.4) in the limit

$$
T \ll \lambda-V(r)
$$

one finds the functional $\tau[\rho]$ in the so-called "low-temperature expansion"

$$
\tau_{\mathrm{ETF}}^{T}[\rho]=\tau_{\mathrm{ETF}}^{T=0}[\rho]+\frac{2 m}{\hbar^{2}} \alpha(\rho) T^{2},
$$

in which the only $T$ dependence comes in through the quadratic term in eq. (6.6). Its coefficient $\alpha(\rho)$ is given by

$$
\alpha(\rho)=\frac{1}{12}\left(3 \pi^{2}\right)^{1 / 3}\left(2 m / \hbar^{2}\right) \rho^{1 / 3} .
$$

The spatial integral of $\alpha(\rho)$ is nothing but the TF approximation to the well-known level density parameter $a_{0}$

$$
a_{0}=\frac{\pi^{2}}{6} \tilde{g}(\lambda),
$$

where $\tilde{g}(\lambda)$ is the average single-particle level density (of one kind of particles).

The functional eq. (6.6) has been used by several authors $[62,104]$ to discuss thermal properties of nuclei. As we also shall demonstrate in the numerical results below, the low- $T$ expansion is a poor approximation when applied to finite nuclei. In the case of a variable effective mass $m^{*}(\boldsymbol{r})=m / f(\boldsymbol{r})$, two more correction terms will arise which remain finite:

$$
\alpha(\rho)=\frac{1}{12}\left(3 \pi^{2}\right)^{1 / 3}\left(\frac{2 m}{\hbar^{2}}\right) \frac{1}{f} \rho^{1 / 3}-\frac{1}{288}\left(3 \pi^{2}\right)^{-1 / 3}\left(\frac{2 m}{\hbar^{2}}\right) \frac{1}{f^{2}} \rho^{-1 / 3}\left[\frac{9}{2} \Delta f-\frac{(\nabla f)^{2}}{f}\right] .
$$

These terms have so far not been taken into account; we have verified, however, that they modify the numerical results only little.

The problem with these relations is that the low-temperature limit eq. (6.5) is only justified in the interior part of the nucleus (or in infinite nuclear matter), where $\lambda-V$ is of the order of $30-40 \mathrm{MeV}$ and the approximation holds up to fairly high temperatures. In the nuclear surface, however, $\lambda-V$ quickly becomes smaller, going through zero at the classical turning point. Thus in the very region where one is interested in going beyond the TF approximation, namely in the surface region, the low- $T$ expansion breaks down. It is therefore not surprising that unsatisfactory results are obtained with the functional (6.6) (see also the numerical results in section 6.3 below).

\subsection{The ETF model at finite temperature*}

Only recently $[106,107]$ the ETF model has been rigorously generalized to finite temperatures,

* In this section, again, we assume only one kind of nucleons. 
treating explicitly the temperature dependence of the gradient corrections up to second order. Since only relatively short presentations have been given in refs. [106,107], we shall resume here the derivation of these gradient corrections.

As in section 3.1, we start with the Wigner-Kirkwood expansion of the Bloch density. To derive the temperature dependence of the latter, we use the fact [17] that the transition from $T=0$ to $T>0$ is formally most easily done by convoluting the single-particle spectral density with the function

$$
f_{T}(E)=\frac{1}{4 T} \frac{1}{\cosh ^{2}(E / 2 T)} .
$$

Thus, due to the convolution theorem, the Bloch density at $T>0$ is a product of the form

$$
C_{T}\left(\boldsymbol{r}, \boldsymbol{r}^{\prime} ; \beta\right)=C_{0}\left(r, \boldsymbol{r}^{\prime} ; \beta\right) \tilde{f}_{T}(\beta),
$$

where $C_{0}\left(r, r^{\prime} ; \beta\right)$ is the Bloch density at $T=0$, given by eq. (3.2), and $\tilde{f}_{T}(\beta)$ is the (two-sided!) Laplace transform of $f_{T}(E)$ (eq. (6.10)):

$$
\tilde{f}_{T}(\beta)=\int_{-\infty}^{+\infty} \mathrm{e}^{-E \beta} f_{T}(E) \mathrm{d} E=\frac{\pi \beta T}{\sin (\pi \beta T)} .
$$

As in eq. (3.3), the inverse Laplace transform of $C_{T}\left(r, r^{\prime} ; \beta\right) / \beta$ now gives the $T>0$ density matrix from which $\rho(r)$ and $\tau(r)$ can be gained analogously to eqs. (3.4), (3.5).

Actually, one does not need to know the off-diagonal part of $C_{T}\left(r, r^{\prime} ; \beta\right)$ for deriving the local densities of interest, as we presently shall show. Let us first introduce a free energy density $\mathscr{F}(r)$ and an entropy density $\sigma(r)$ by

$$
\begin{aligned}
& F=\int \mathrm{d}^{3} r \mathscr{F}(r)=\sum_{\nu} \varepsilon_{\nu} n_{\nu}-T S, \\
& S=\int \mathrm{d}^{3} r \sigma(r),
\end{aligned}
$$

where $S$ is the entropy (eq. (6.2)), and $F$ the single-particle free energy (each for one kind of nucleons). It is now a direct consequence of a relation derived in ref. [17] that the free energy density $\mathscr{F}(r)$ can be obtained from the local Bloch density by

$$
\mathscr{F}(\boldsymbol{r})=\lambda \rho(r)-\mathscr{L}_{\lambda}^{-1}\left[\frac{1}{\beta^{2}} C_{T}(r, \beta)\right]
$$

The entropy density is then given by

$$
\sigma(r)=-\frac{\partial}{\partial T} \mathscr{F}(r)=\frac{\partial}{\partial T} \mathscr{L}_{\lambda}^{-1}\left[\frac{1}{\beta^{2}} C_{T}(r, \beta)\right] .
$$


Through the Schrödinger equation which defines the spectrum $\varepsilon_{i}$, we can now also obtain the kinetic energy density:

$$
\begin{aligned}
\frac{\hbar^{2}}{2 m} \tau(r) & =\frac{\hbar^{2}}{2 m} \sum_{\nu}\left|\nabla \varphi_{\nu}(r)\right|^{2} n_{\nu} \\
& =\mathscr{F}(r)-V(r) \rho(r)+T \sigma(r)+\frac{1}{2} \frac{\hbar^{2}}{2 m} \Delta \rho(r) .
\end{aligned}
$$

(Here we have made use of the relation

$$
\tau(r)=-\sum_{\nu} \varphi_{\nu}^{*} \Delta \varphi_{\nu} n_{\nu}+\frac{1}{2} \Delta \rho
$$

which holds for systems with time reversal symmetry.) Equations (6.15)-(6.17) together with the expression for the local density

$$
\rho(r)=\sum_{\nu}\left|\varphi_{\nu}(r)\right|^{2} n_{\nu}
$$

allow to calculate all single-particle quantities of interest from the local Bloch density. Note that these equations are exact, as long as the exact Bloch density $C_{0}(r, \beta)$ according to eq. (3.2) is used in eq. (6.11).

To obtain the semiclassical ETF expressions for these densities, it is sufficient now to replace $C_{0}$ in eq. (6.11) by its Wigner-Kirkwood expansion, eq. (3.8), and to leave the factor $\tilde{f}_{T}(\beta)$ untouched. (If we would expand the latter in powers of $\beta T$, we would obtain the low- $T$ expansion.) After the Laplace inversions term by term, we obtain for a local potential $V(\boldsymbol{r})$ the following expressions up to order $\hbar^{2}$ :

$$
\begin{aligned}
\rho_{\mathrm{ETF}}^{T}(\boldsymbol{r})= & \frac{1}{2 \pi^{2}}\left(\frac{2 m}{\hbar^{2}}\right)^{3 / 2}\left\{T^{3 / 2} J_{1 / 2}(\eta)+\frac{1}{24} \frac{\hbar^{2}}{2 m}\left[\frac{3}{4} T^{-3 / 2} J_{-5 / 2}(\eta)(\nabla V)^{2}+T^{-1 / 2} J_{-3 / 2}(\eta) \Delta V\right]\right\} \\
\tau_{\mathrm{ETF}}^{T}(r)= & \frac{1}{2 \pi^{2}}\left(\frac{2 m}{\hbar^{2}}\right)^{5 / 2}\left\{T^{5 / 2} J_{3 / 2}(\eta)-\frac{1}{4} \frac{\hbar^{2}}{2 m}\left[\frac{3}{8} T^{-1 / 2} J_{-3 / 2}(\eta)(\nabla V)^{2}+\frac{5}{6} T^{1 / 2} J_{-1 / 2}(\eta) \Delta V\right]\right\} \\
\mathscr{F}_{\mathrm{ETF}}(r)= & \lambda \rho_{\mathrm{ETF}}^{T}(\boldsymbol{r})-\frac{1}{2 \pi^{2}}\left(\frac{2 m}{\hbar^{2}}\right)^{3 / 2}\left\{\frac{2}{3} T^{5 / 2} J_{3 / 2}(\eta)-\frac{1}{12} \frac{\hbar^{2}}{2 m}\right. \\
& \left.\times\left[\frac{1}{4} T^{-1 / 2} J_{-3 / 2}(\eta)(\nabla V)^{2}+T^{1 / 2} J_{-1 / 2}(\eta) \Delta V\right]\right\} \\
\sigma_{\mathrm{ETF}}(r)= & \eta \rho_{\mathrm{ETF}}^{T}(r)+\frac{1}{2 \pi^{2}}\left(\frac{2 m}{\hbar^{2}}\right)^{3 / 2}\left\{\frac{5}{3} T^{3 / 2} J_{3 / 2}(\eta)\right. \\
& \left.+\frac{1}{24} \frac{\hbar^{2}}{2 m}\left[\frac{1}{4} T^{-3 / 2} J_{-3 / 2}(\eta)(\nabla V)^{2}-T^{-1 / 2} J_{-1 / 2}(\eta) \Delta V\right]\right\}
\end{aligned}
$$


where

$$
\eta=\{\lambda-V(\boldsymbol{r})\} / T
$$

$J_{\mu}(\eta)$ are generalizations of the so-called Fermi integrals, which for $\mu>-1$ are defined by

$$
J_{\mu}(\eta)=\int_{0}^{\infty} \frac{x^{\mu}}{1+\exp (x-\eta)} \mathrm{d} x \quad(\mu>-1) .
$$

For $\mu<-1$, where the integral (6.25) does not exist, we use the derivative recursion relation

$$
J_{\mu}(\eta)=\frac{1}{(\mu \pm 1)} \frac{\mathrm{d}}{\mathrm{d} \eta} J_{\mu+1}(\eta) \quad(\mu \neq-1)
$$

as a definition. This leads to well-behaved, continuous functions $J_{\mu}(\eta)$ for all values of $\mu$ occurring in this context. We do therefore not encounter here the turning point problem known in the $T=0$ case (see section 3): all the densities, eqs. (6.20)-(6.23), are continuous and finite everywhere in space. Only in the limit $T=0$, the semiclassical corrections diverge as one approaches the classical turning points from the inside region (i.e. in the limit $\eta \rightarrow+\infty)$. The leading order terms in eqs. (6.20)-(6.23) are, of course, the well-known TF relations [101, 102, 108].

As in the $T=0$ case, we now eliminate the gradients of $V$ from the above expressions. This procedure is described in ref. [106]. As a result, we obtain the following kinetic energy and entropy density functionals:*

$$
\begin{aligned}
& \tau_{\text {TETF }}[\rho]=\tau_{\mathrm{TF}}^{T}[\rho]+\tau_{2}^{T}[\rho], \\
& \sigma_{\mathrm{TETF}}[\rho]=\sigma_{\mathrm{TF}}[\rho]+\sigma_{2}[\rho] .
\end{aligned}
$$

The TF terms have the familiar form $[101,102,108]$

$$
\begin{aligned}
& \tau_{\mathrm{TF}}^{T}[\rho]=\frac{2 m}{\hbar^{2}} A_{T} T J_{3 / 2}(\tilde{\eta}), \\
& \sigma_{\mathrm{TF}}[\rho]=\frac{5}{3} A_{T} J_{3 / 2}(\tilde{\eta})-\tilde{\eta} \rho,
\end{aligned}
$$

in terms of the quantity $\tilde{\eta}$ which for a given $T$ is the (unique) solution of the equation

$$
\rho=A_{T} J_{1 / 2}(\tilde{\eta})
$$

with

$$
A_{T}=\frac{1}{2 \pi^{2}}\left(\frac{2 m}{\hbar^{2}}\right)^{3 / 2} T^{3 / 2}
$$

Note that $\tilde{\eta}$ is an explicit function of $T$ and $\rho: \tilde{\eta}(\rho, T)$.

* See ref. [112] for the derivation of the fourth order gradient terms. 
The second order corrections are

$$
\begin{aligned}
& \tau_{2}^{T}[\rho]=\gamma(\tilde{\eta}) \frac{(\nabla \rho)^{2}}{\rho}+\frac{1}{3} \Delta \rho \\
& \sigma_{2}[\rho]=-\nu(\tilde{\eta}) \frac{(\nabla \rho)^{2}}{\rho} \frac{1}{T} \frac{\hbar^{2}}{2 m}
\end{aligned}
$$

where

$$
\begin{aligned}
& \gamma(\tilde{\eta})=\zeta(\tilde{\eta})-\nu(\tilde{\eta}) \\
& \zeta(\tilde{\eta})=-\frac{J_{1 / 2}(\tilde{\eta}) J_{-3 / 2}(\tilde{\eta})}{12 J_{-1 / 2}^{2}(\tilde{\eta})} \equiv-\frac{1}{6} J_{1 / 2}(\tilde{\eta}) \frac{\mathrm{d}}{\mathrm{d} \tilde{\eta}}\left[\frac{1}{J_{-1 / 2}(\tilde{\eta})}\right] \\
& \nu(\tilde{\eta})=-\frac{3}{2} \zeta(\tilde{\eta})+36[\zeta(\tilde{\eta})]^{2}-\left.\frac{3}{8} \frac{J_{1 / 2}^{2}(\tilde{\eta}) J_{-5 / 2}(\tilde{\eta})}{J_{-1 / 2}^{3}(\tilde{\eta})} \equiv T \frac{\partial}{\partial T} \zeta(\tilde{\eta})\right|_{\rho}
\end{aligned}
$$

The form of the Weizsäcker term is thus still the same but its coefficient depends on $T$ and $\rho$ (see fig. 13 below).

The inclusion of a variable effective nuclear mass and a spin-orbit potential does not create any new problems and can, with some algebraic effort, be done straightforwardly up to second order [106]. Starting from a Skyrme type one-body Hamiltonian, eq. (3.19), one finds that the TF relations eqs. (6.29)-(6.32) remain the same if the mass $m$ is replaced everywhere by the effective mass $m^{*}(r)=$ $m / f(r)$. The total TF free energy density then becomes (for one kind of particles)

$$
\mathscr{F}_{\mathrm{TF}}[\rho]=-\frac{2}{3} T A_{T}^{*} J_{3 / 2}(\tilde{\eta})+T \tilde{\eta} \rho+\mathscr{E}_{\mathrm{pot}}^{\prime}[\rho]
$$

with

$$
A_{T}^{*}=\frac{1}{2 \pi^{2}}\left(\frac{2 m}{\hbar^{2}}\right)^{3 / 2} f^{-3 / 2} T^{3 / 2}
$$

where $f$ is, of course, a functional of $\rho$, too (section 2.1). The quantity $\tilde{\eta}$ must thus be calculated from eq. (6.31) with $A_{T}$ replaced by $A_{T}^{*}$ and taking the $\rho$ dependence of $f$ into account. The quantity $\mathscr{E}_{\text {pot }}^{\prime}[\rho]$ in eq. (6.38) is the potential energy density $V \rho$ in the case of a fixed external potential. In the selfconsistent case with a Skyrme interaction, it is to be taken as the sum of all potential energy contributions except those proportional to $\tau \rho$; these are already included in the kinetic energy term in eq. (6.38).

The second-order gradient correction to the free energy density (including all spin-orbit contributions) becomes

$$
\begin{aligned}
\mathscr{F}_{2}[\rho]= & \frac{\hbar^{2}}{2 m}\left\{\zeta(\tilde{\eta}) f \frac{(\nabla \rho)^{2}}{\rho}+\left[\frac{9}{4} \zeta(\tilde{\eta})-\frac{7}{48}\right] \rho \frac{(\nabla f)^{2}}{f}\right. \\
& \left.+\frac{1}{6}(\rho \Delta f-f \Delta \rho)+\left[3 \zeta(\tilde{\eta})-\frac{5}{12}\right] \nabla \rho \cdot \nabla f\right\}-\frac{1}{2}(W)^{2} \frac{2 m}{\hbar^{2}} \frac{1}{f} \rho
\end{aligned}
$$


(Note that the spin-orbit contributions, to this order, keep the same form as in the $T=0$ case.) The functionals $\tau_{2}^{T}[\rho]$ and $\sigma_{2}[\rho]$ can be obtained from eq. (6.40) using the canonical relation $\sigma_{2}[\rho]=$ $-(\partial / \partial T) \mathscr{F}_{2}[\rho]$ and an expression analogous to eq. (6.17). Hereby it is useful to note that

$$
\frac{\partial}{\partial T} \zeta(\tilde{\eta})=\frac{1}{T} \nu(\tilde{\eta}), \quad(T>0)
$$

where the partial derivative $\partial / \partial T$ is taken at $\rho=$ const. The coefficient $\zeta(\tilde{\eta})$ which appears in $\mathscr{F}_{2}[\rho]$ (eq. (6.40)) can be approximated to within less than $3 \%$ for all values of $\tilde{\eta}$ by the simple form

$$
\zeta(\tilde{\eta}) \approx \frac{1}{36}\left[1+2 / \sqrt{1+\mathrm{e}^{\bar{\eta}}}\right] .
$$

It is now of special interest to discuss the temperature dependence of the coefficients $\zeta, \gamma$ and $\nu$, and in particular to study the limit $T \rightarrow 0$ of the above functionals. The key quantity for this investigation is the parameter $\tilde{\eta}$, defined through eq. (6.31) by the density $\rho$. If $\tilde{\eta}$ is much larger than unity, one can use the following asymptotic expansion of the $J_{\mu}(\eta)$ [108]:

$$
J_{\mu}(\eta)=\frac{1}{\mu+1} \eta^{\mu+1}\left[1+\mu(\mu+1) \frac{\pi^{2}}{6} \eta^{-2}+\mathcal{O}\left(\eta^{-4}\right)+\cdots\right] \quad(\eta \gg 1)
$$

We then get for the density from eq. (6.31)

$$
\rho=\frac{1}{3 \pi^{2}}\left(\frac{2 m}{\hbar^{2}}\right)^{3 / 2} T^{3 / 2} \tilde{\eta}^{3 / 2}\left[1+\frac{\pi^{2}}{8} \tilde{\eta}^{-2}+\cdots\right] \quad(\tilde{\eta} \gg 1) .
$$

In the nuclear interior, where (for one kind of nucleons) $\rho \geq 0.06 \mathrm{fm}^{-3}$, we find at $T=1 \mathrm{MeV}$ that $\tilde{\eta} \geq 30$, so that the expansion (6.43) is well justified. For a fixed value of $\rho, \tilde{\eta}$ will become even larger if $T$ decreases, and in the limit $T \rightarrow 0, \tilde{\eta}$ will go to infinity like $1 / T$.

The crucial point is now to realize [109] that this happens for any finite value of $\rho$. This is so, because $J_{1 / 2}(\tilde{\eta})$ is a monotonously increasing function of $\tilde{\eta}$. Thus, even if we choose a small value of $\rho$ so that for an initial value of $T, \tilde{\eta}$ turns out to be of order unity or even negative and the expansion (6.43) cannot be used, $\tilde{\eta}$ will increase with decreasing $T$ (at fixed $\rho$ ), until it eventually becomes so large that eq. (6.44) becomes valid. Thus, in the limit $T \rightarrow 0$, the first term of eq. (6.44) will always be dominant with $\tilde{\eta}$ tending towards infinity. At the same time, $\tau_{\mathrm{TF}}^{T}[\rho]$ (eq. (6.29)) will go like $(T \tilde{\eta})^{5 / 2}$ and eliminating $T \tilde{\eta}$, we recover the old TF relation eq. (3.14). In the same way $\sigma_{\mathrm{TF}}[\rho]$ is found to go to zero like $T$. For the coefficients of the gradient corrections, we find the limits

$$
\left.\begin{array}{r}
\gamma(\tilde{\eta}) \text { and } \zeta(\tilde{\eta}) \underset{T \rightarrow 0}{\longrightarrow} \frac{1}{36}, \\
\nu(\tilde{\eta}) \underset{T \rightarrow 0}{\longrightarrow} 0 \text { like } T^{2}
\end{array}\right\}(\tilde{\eta} \rightarrow \infty)
$$

We thus recover also the familiar coefficient $1 / 36$ of the Weizsäcker term in $\tau_{2}^{T}[\rho]$ (eq. (6.33)), and $\sigma_{2}[\rho]$ goes to zero as it should. 
Note that the above arguments do not depend on the point in space where we picked our density $\rho$. We thus come to the surprising result [109] that for $T=0$, the old functional $\tau_{\mathrm{ETF}}[\rho]$ is correct everywhere in space, even in the classically forbidden region! This result holds also if the effective mass is included as in eqs. (6.38)-(6.40). It can also be extended to the fourth order corrections $\tau_{4}[\rho]$ (see section 3.2 and appendix A) even though the explicit form of $\tau_{4}[\rho]$ at $T>0$ has not been derived yet, ${ }^{*}$ since in the limit $\tilde{\eta} \rightarrow \infty$ the way of constructing these functionals becomes identical to that at $T=0$ inside the classically allowed region; the essential relationship (6.31) between $\tilde{\eta}$ and $\rho$ is not changed if the 4th order terms are added.

In order to illustrate the above points, we show in fig. 13 the coefficients $\gamma$ and $\nu$ for various temperatures, plotted along with typical density profiles of a spherical nucleus, as functions of the radial variable $r$. (The profiles $\rho(r)$ are those obtained selfconsistently for the $N=146$ neutrons of ${ }^{240} \mathrm{Pu}$; below $T=1 \mathrm{MeV}$, their parameters do not change with decreasing $T$.) We see that already for $T \leqslant 1 \mathrm{MeV}$, the limiting values of eq. (6.45) are approximately reached throughout the major part of the nucleus. Only in the outer surface, $\gamma$ and $\nu$ start varying. After reaching a maximum value of $\nu_{0}=0.02494$ which is independent of $T$ (since $\nu(\tilde{\eta})$ is a universal function, see eq. (6.37)), $\nu$ drops again to zero at even larger distances. On the other hand, $\gamma$ approaches a new constant value $1 / 12$ in the extreme outer surface. These limiting values can be found from eqs. (6.35)-(6.37) taking the limit $\tilde{\eta} \rightarrow-\infty$ and using the asymptotic series of the $J_{\mu}(\eta)[108]$

$$
J_{\mu}(\eta)=\Gamma(\mu+1) \sum_{k=1}^{\infty}(-1)^{k-1} \frac{1}{k^{\mu+1}} \mathrm{e}^{k \eta} \quad(\eta<0)
$$

The entropy density correction $\sigma_{2}[\rho]$ (eq. (6.34)), which is proportional to $\nu$, picks up contributions only from the outer surface of the nucleus (except at temperatures $T \geq 1-2 \mathrm{MeV}$ ). The maximum in the

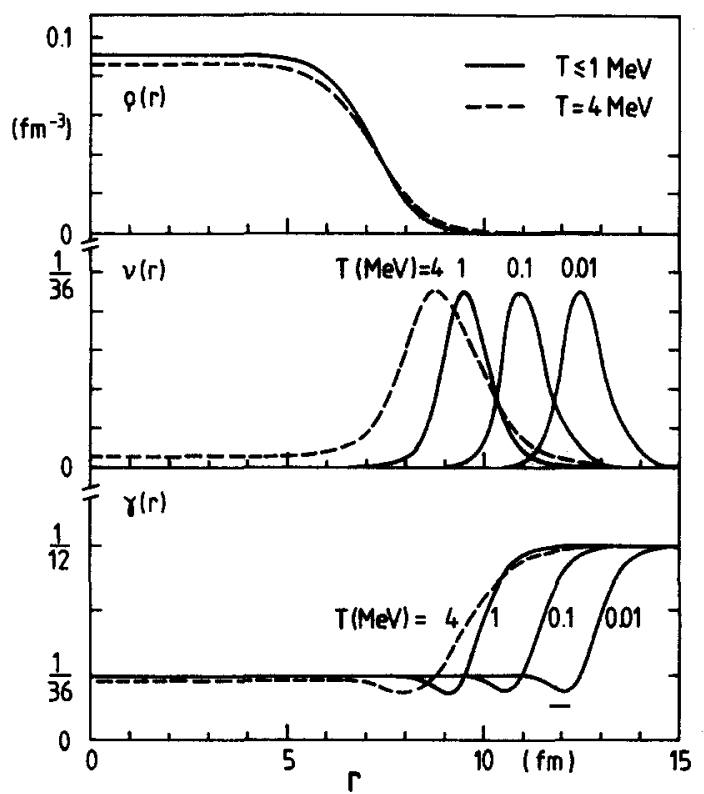

Fig. 13. Radial dependence of a typical density profile and the coefficients $\nu(6.37)$ and $\gamma(6.35)$ at various temperatures $T$. Dashed curves are for $T=4 \mathrm{MeV}$; the solid curves for $\rho(r)$ hold for all temperatures $T \leqq 1 \mathrm{MeV}$.

* See, however, ref. [112]. 
curve $\nu(r)$-which is centred near $\tilde{\eta} \approx 0^{*}$ - thus divides the space into two regions: the one inside the nucleus, where $\tilde{\eta} \gg 1$ and the expansion (6.43) holds, and the outer region where $\tilde{\eta}<0$ and the expansion (6.46) may be used. In the latter domain, we get for the density

$$
\rho \simeq A_{T} \frac{\sqrt{\pi}}{2} \mathrm{e}^{\tilde{\eta}} \quad(\tilde{\eta} \ll 0),
$$

so that $\tilde{\eta}$ goes to $-\infty$ like $\ln \rho$ with $\rho \rightarrow 0$ for a finite fixed temperature $T$. This might seem to contradict the above discussion about the $T \rightarrow 0$ limit. However, one should be very careful not to confuse the two limiting processes: $T \rightarrow 0$ at fixed $\rho>0$ on one hand, leading to the proof of the validity of the $T=0$ functional $\tau_{\text {ETF }}[\rho]$, and $\rho \rightarrow 0$ at fixed $T>0$ on the other hand. This latter limit is rather academic, since eq. (6.47) only becomes valid at far distances from the nuclear surface which are of little physical interest. (The fact that in an equilibrium situation under pressure at $T>0$, the density $\rho$ will never become zero but takes a finite gas value, will be discussed in section 6.3 below.)

In refs. $[106,107]$, the point where $\tilde{\eta}=0$ was erroneously identified with the classical turning point (the two points happen to be close to each other for $T \approx 1-2 \mathrm{MeV}$ !), misleading us to the statement that the functional $\tau_{\text {ETF }}[\rho]$ is not analytical at the turning point for $T=0$. This is however not true, as we have explained above (see also ref. [109]).**

Let us summarize the two important points of this section:

1) The ETF functionals $\tau[\rho]$ and $\sigma[\rho]$ have been derived up to second order gradient terms for finite temperatures $T$. The gradient corrections contain the functions $\nu(\tilde{\eta}), \zeta(\tilde{\eta})$ and $\gamma(\tilde{\eta})$, which can be computed once for all in terms of the functions $J_{\mu}(\tilde{\eta})$, and which by means of eq. (6.31) introduce implicitly a temperature dependence. The fourth order terms are found in ref. [112].

2) By taking a careful limit $T \rightarrow 0$ for a fixed $\rho$, one can show that the old functional $\tau_{E T F}[\rho]$ at $T=0$, which so far only could be derived inside the classically allowed region, actually is correct in the whole space.

\subsection{Discussion of the ETF Euler equation at $T>0$}

In this section we shall discuss the Euler equation derived from the new ETF functionals at $T>0$, given by eqs. (6.27)-(6.37). We shall again simplify the discussion assuming only one kind of nucleons and neglecting effective mass, spin-orbit and Coulomb contributions.

A hot, isolated nucleus is not stable since it evaporates nucleons, and cannot therefore be described in a static variational calculation. However, assuming the evaporation time to be much larger than the equilibration time, we may consider such a nucleus as a metastable system, much like an overheated classical liquid drop under tension [110]. This case has been discussed in the TF approximation by Stocker and Burzlaff [101].

On the other hand, in the context of stellar collapse in astrophysics, one speaks of nuclei in a thermal equilibrium with a gas of nucleons and leptons at finite temperatures and pressures [22, 23]. We shall therefore discuss the Euler equation including an external pressure which later may be switched off according to the required boundary conditions.

In the presence of a constant external pressure $P_{0}$ one has to minimize the Gibbs free energy, thus

\footnotetext{
- More precisely, $\nu$ takes its maximum value $\nu_{0}$ at $\bar{\eta}_{0}=1.358$.

${ }^{* *}$ Note that in refs. $[106,107]$, the quantity $\nu(\tilde{\eta})($ eq. $(6.37))$ was erroneously quoted with the wrong overall sign.
} 
writing

$$
\delta \int \mathrm{d}^{3} r\left\{\mathscr{F}[\rho]-\lambda \rho(\boldsymbol{r})+P_{0}\right\}=0
$$

In calculations of the equation of state of hot nuclear matter pertinent to astrophysical applications, one usually divides the space into Wigner-Seitz cells, containing each one nucleus, and imposes periodic boundary conditions $[23,114]$. We shall in the following only consider one cell, assuming it to be large enough so that the nucleon density reaches always its free gas solution at the boundaries.

We may separate the free energy density $\mathscr{F}[\rho]$ in eq. (6.48) into an "infinite" and a surface part

$$
\mathscr{F}[\rho]=\mathscr{F}_{\infty}(\rho)+b(\nabla \rho)^{2}+\mathscr{F}_{2}[\rho]
$$

where $\mathscr{F}_{\infty}[\rho]$ is given by the TF expression eq. (6.38); the second term in eq. (6.49) contains the gradient contributions to the Skyrme potential energy density proportional to $t_{1}$ and $t_{2}$, see eqs. (2.5), (4.11), and the last term includes the second order gradient corrections $\tau_{2}[\rho]$ and $\sigma_{2}[\rho]$. The variational equation (6.48) then becomes (irrespectively of the value of $P_{0}$ )

$$
\frac{\mathrm{d}}{\mathrm{d} \rho} \mathscr{F}_{\infty}(\rho)-2 b \Delta \rho+\frac{\delta \mathscr{F}_{2}[\rho]}{\delta \rho}=\lambda .
$$

To be realistic, we should also include a fourth order term $\mathscr{F}_{4}[\rho]$ which, however, is not known* for $T>0$. Nevertheless we think that the discussion of eq. (6.50) is of some value, in particular also since the nature of its solutions at $T>0$ is already determined at the pure TF level and does not change qualitatively when the $\mathscr{F}_{2}[\rho]$ contribution is included (contrary to the case at $T=0$ discussed in section 3.3).

From eqs. (6.26), (6.31) and (6.38) with $m^{*}=m$ (i.e., $f \equiv 1$ ), we find explicitly for the variation of the bulk free energy

$$
\frac{\mathrm{d}}{\mathrm{d} \rho} \mathscr{F}_{\infty}(\rho)=T \tilde{\eta}+\frac{\mathrm{d}}{\mathrm{d} \rho} \mathscr{E}_{\mathrm{pot}}^{\infty}(\rho),
$$

where $\mathscr{E}_{\text {pot }}^{\infty}(\rho)$ contains only the "volume" parts proportional to $t_{0}$ and $t_{3}$ of the Skyrme energy density. Note that in deriving eq. (6.51), two terms proportional to $\mathrm{d} \tilde{\eta} / \mathrm{d} \rho$ cancel identically (also for $f \neq$ const.). Calculating the variation of $\mathscr{F}_{2}[\rho]$, we note that

$$
\left.\frac{\mathrm{d}}{\mathrm{d} \rho} \zeta(\tilde{\eta})\right|_{T=\text { const. }}=-\frac{2}{3} \frac{\nu(\tilde{\eta})}{\rho}
$$

and obtain

$$
\frac{\delta \mathscr{F}_{2}[\rho]}{\delta \rho}=\frac{\hbar^{2}}{2 m}\left[\left(\zeta+\frac{2}{3} \nu\right) \frac{(\nabla \rho)^{2}}{\rho^{2}}-2 \zeta \frac{\Delta \rho}{\rho}\right]
$$

In order to further simplify the discussion, we neglect curvature and compression effects and consider

*It now is known, see ref. [112]. 
the geometry of semi-infinite nuclear matter described by a 1-dimensional profile $\rho(z)$. (The finite size effects will be estimated later on in section 6.5 in a leptodermous expansion of the free energy, similarly as in section 5 for the $T=0$ case.) We thus arrive at the Euler equation

$$
\frac{\mathrm{d}}{\mathrm{d} \rho} \mathscr{F}_{\infty}-2 b \rho^{\prime \prime}(z)+\frac{\hbar^{2}}{2 m}\left[\left(\zeta+\frac{2}{3} \nu\right)\left(\frac{\rho^{\prime}}{\rho}\right)^{2}-2 \zeta \frac{\rho^{\prime \prime}}{\rho}\right]=\lambda
$$

Using the substitution $\rho^{\prime}(z)=y(\rho)$, eq. (6.54) can be integrated once to yield

$$
\left(\frac{\mathrm{d} \rho}{\mathrm{d} z}\right)^{2}\left[b+\frac{1}{\rho} \frac{\hbar^{2}}{2 m} \zeta(\tilde{\eta})\right]=\mathscr{F}_{\infty}(\rho)-\lambda \rho+P_{0}
$$

where we have identified the integration constant with the external pressure $P_{0}$ appearing in eq. (6.48). For a given force, eq. (6.55) can easily be integrated numerically to yield the inverse profile function $z(\rho)$ :

$$
z(\rho)=-\int_{\rho 1}^{\rho} \sqrt{\frac{b+\left(1 / \rho^{\prime}\right) \zeta\left(\tilde{\eta}^{\prime}\right)}{\Phi_{\infty}\left(\rho^{\prime}\right)-\lambda \rho^{\prime}+P_{0} / 2 m}} \mathrm{~d} \rho^{\prime} .
$$

Equation (6.56) shall be used in section 6.5 in numerical calculations of liquid-drop model parameters for the semi-infinite case.

The qualitative solution for $\rho(z)$ depends on the boundary conditions imposed by the choice of $P_{0}$. We shall discuss separately in the following the two cases $P_{0}=0$ and $P_{0} \neq 0$.

\subsubsection{Case of a free nucleus $\left(P_{0}=0\right)$}

Let us first consider the case of an isolated nucleus where $P_{0}=0$. The Euler equation (6.50) has been discussed both without and with inclusion of a Weizsäcker type gradient correction in refs. [101] and [104], respectively. (In the latter case, a constant phenomenological value of $\zeta$ was used and the entropy correction $\sigma_{2}[\rho]$ was neglected.) As shown in both cases, one cannot find any solution which goes asymptotically to a constant (finite or zero) density outside the surface; this holds for any finite temperature.

We can see this immediately from eqs. (6.50) and (6.55). Looking for solutions with constant density, eq. (6.50) becomes simply

$$
\frac{\mathrm{d}}{\mathrm{d} \rho} \mathscr{F}_{\infty}(\rho)=\lambda
$$

on the other hand, eq. (6.55) becomes (with $P_{0}=0$ )

$$
F_{\infty}(\rho)=\lambda \rho \text {. }
$$

The two above equations can, for any finite temperature $T$, only be fulfilled for one value $\rho_{0}$ of the density, namely for the saturation point where the curve $F_{\infty}(\rho) / \rho$ has its minimum. This point $\rho_{0}$ corresponds to zero pressure: 


$$
P\left(\rho_{0}\right)=\rho_{0}^{2} \frac{\mathrm{d}}{\mathrm{d} \rho}\left[\frac{F_{\infty}(\rho)}{\rho}\right]_{\rho_{0}}=\rho_{0} \mathscr{F}_{\infty}^{\prime}\left(\rho_{0}\right)-\mathscr{F}_{\infty}\left(\rho_{0}\right)=0
$$

The density $\rho_{0}$ thus corresponds to the asymptotic value reached in the nuclear interior (apart from compression effects). Note that $\rho=0$ is no solution of eqs. $(6.57,58)$ since $\mathrm{d} \mathscr{F}_{\infty} / \mathrm{d} \rho$ tends to $-\infty$ for $\rho \rightarrow 0$ at finite temperature, as follows from the discussion after eq. (6.47) and from eq. (6.51).

Integrating eq. (6.55) from the interior limit $\rho_{0}$ outwards through the surface, one finds [101, 104] a point $z_{0}$ at which the density has a zero slope, but nonvanishing zeroth and second derivatives:

$$
\rho^{\prime}\left(z_{0}\right)=0 ; \quad \rho\left(z_{0}\right)=\rho_{\mathrm{g}} ; \quad \rho^{\prime \prime}\left(z_{0}\right)=c_{0}>0
$$

Beyond this minimum, the density raises again quadratically; it has therefore to be cut at $z=z_{0}$ in order to be a physically acceptable solution, as illustrated in fig. 14. This somewhat funny behaviour of the surface may seem unsatisfactory at the first glance; it must, however, be interpreted [101] as an expression of the fact that the nuclear system is metastable at $T>0$.

The density $\rho_{\mathrm{g}}$ reached at the minimum is also a solution of eq. (6.58), as follows from eq. (6.55); it does, however, not satisfy eq. (6.57). In fact, the difference $\mathrm{d} \mathscr{F}_{\infty} /\left.\mathrm{d} \rho\right|_{\rho=\rho_{\mathrm{g}}}-\lambda$ is due to the second order derivatives $\rho^{\prime \prime}\left(z_{0}\right)$ occurring in the Euler equation (6.54), from which we can determine the value of $c_{0}$ :

$$
c_{0}=\rho^{\prime \prime}\left(z_{0}\right)=\frac{P_{\mathrm{g}}}{\left[2 b \rho_{\mathrm{g}}+2 \zeta_{\mathrm{g}} \hbar^{2} / 2 m\right]}
$$

Here $P_{\mathrm{g}}$ is the pressure of a nucleon gas at density $\rho_{\mathrm{g}}$ :

$$
P_{\mathrm{g}}=\rho_{\mathrm{g}} \mathscr{F}_{\infty}^{\prime}\left(\rho_{\mathrm{g}}\right)-\mathscr{F}_{\infty}\left(\rho_{\mathrm{g}}\right)>0
$$

and $\zeta_{\mathrm{g}}=\zeta\left(\tilde{\eta}\left(\rho_{\mathrm{g}}\right)\right)$.

This qualitative behaviour does not depend on the presence of the second order gradient corrections (the precise value of $c_{0}$ (eq. (6.61)) will, of course, depend on it). It is also found for a finite nucleus in 3 dimensions; eq. (6.61) then gives the curvature of the radial profile at the finite distance $R_{0}$ where the minimum occurs: $c_{0}=\rho^{\prime \prime}\left(R_{0}\right)=\Delta \rho\left(R_{0}\right)$.

In fig. 15 we show the limiting densities $\rho_{0}$ and $\rho_{\mathrm{g}}$ as functions of the temperature (dashed-dotted

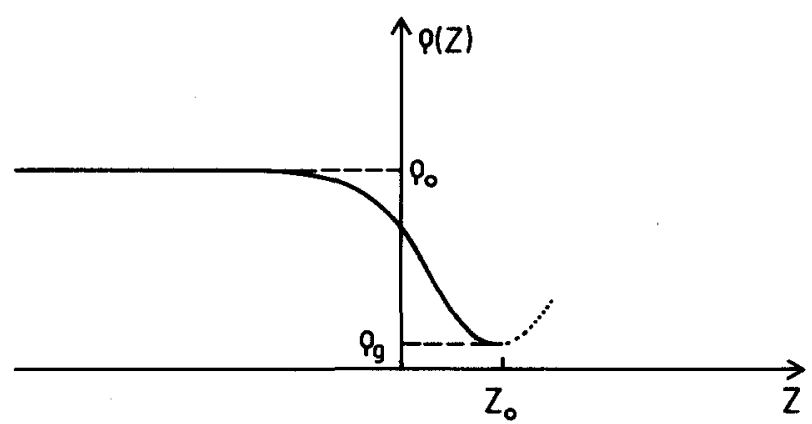

Fig. 14. Schematized density profile of a very large metastable nucleus (or semi-infinite nuclear matter) at finite temperature without external pressure. The profile $\rho(z)$ is quadratic around the minimum at $z=z_{0}$. 


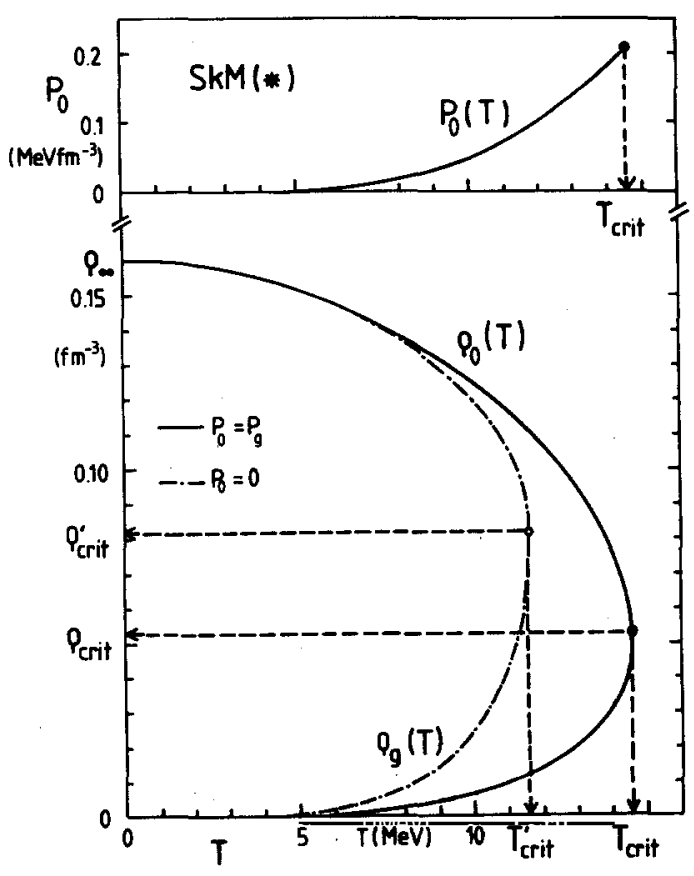

Fig. 15. Infinite symmetric nuclear matter densities for the SkM or $S k M^{*}$ force versus temperature $T$. $\rho_{0}$ : condensed (liquid) phase, $\rho_{k}$ : gas phase. Solid lines: both phases in thermal equilibrium at finite pressure $\left(P_{0}=P_{s}\right.$ shown on the top). Dashed-dotted lines: zero pressure in condensed phase $\left(P_{0}=0\right)$ but finite pressure $P_{8}>0$ in gas phase; corresponding to metastable situation illustrated in fig. 14.

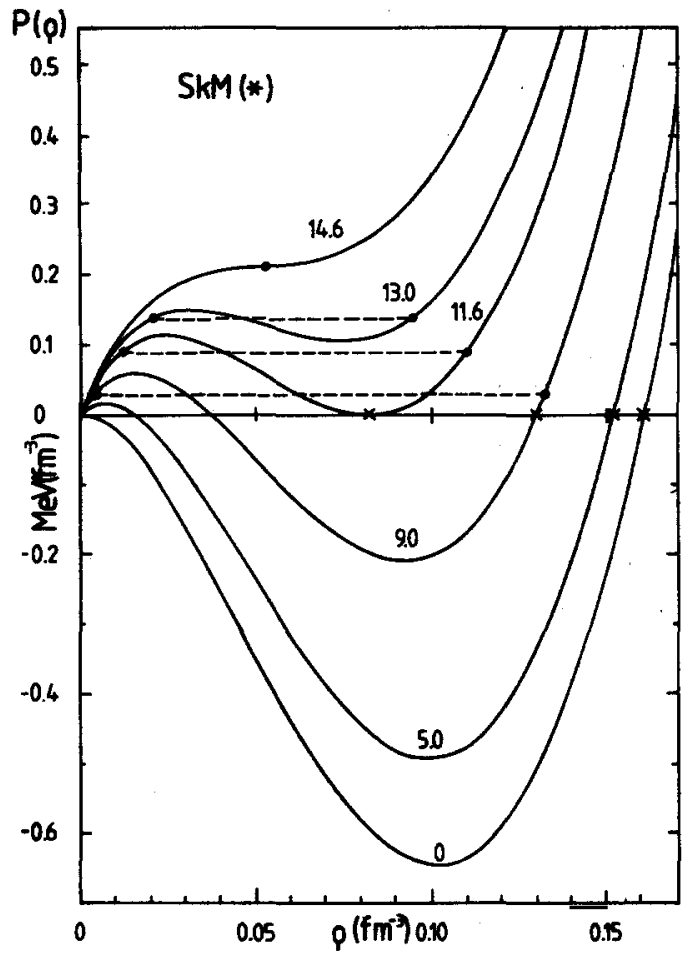

Fig. 16. Pressure versus density isotherms of symmetric infinite nuclear matter obtained with the SkM or the SkM* force. The temperature is indicated by numbers (in MeV). Crosses: saturation densities $\left(P_{0}=0\right)$. Dots connected by dashed horizontal lines: gas and liquid phase densities $\left(\rho_{\mathrm{g}}\right.$ and $\rho_{0}$, resp.) for thermodynamical equilibrium with $P_{0}=P_{2}>0$. Note in particular the curves for the critical temperatures $T_{\text {crit }}^{\prime}=11.6 \mathrm{MeV}$ and $T_{\text {crit }}=14.6 \mathrm{MeV}$.

lines, according to $P_{0}=0$ ). At a critical temperature $T_{\text {crit }}^{\prime}$, they become equal: $\rho_{0}=\rho_{\mathrm{g}}=\rho_{\text {crit }}^{\prime}$. Note that this critical temperature is lower than that $\left(T_{\text {crit }}\right)$ of two infinite nuclear phases at pressure equilibrium which we will discuss below. $T_{\text {crit }}^{\prime}$ corresponds thus to the limiting temperature for a very heavy isolated nucleus (we have neglected the curvature and compression effects!) with $N=Z$ and without Coulomb interaction. (The Coulomb interaction will of course decrease its stability and thus also $T_{\text {crit }}^{\prime}$.) Near $T=0$, the inner density $\rho_{0}(T)$ decreases quadratically from the saturation value $\rho_{\infty}$ at $T=0$. The density $\rho_{\mathrm{g}}(T)$ goes exponentially to zero with $T$ according to eq. (6.47) which turns out to be approximately true for the limiting gas density $\rho_{\mathrm{g}}$ at $T \leqslant 5-6 \mathrm{MeV}$.*

In fig. 16 we show isotherms of the pressure as function of the density of symmetric infinite nuclear matter. Both figs. 15 and 16 were obtained with the Skyrme forces SkM or SkM* (they are identical for symmetric infinite nuclear matter). The crosses on the axis $P=0$ correspond to the values of $\rho_{0}$. We see that $T_{\text {crit }}^{\prime} \simeq 11.6 \mathrm{MeV}$ is the highest temperature for which the curve $P(\rho)$ touches the abscissa for a finite value of $\rho$.

* Note, however, that the eq. (6.61) does not bold at $T=0$; see section 3.3 for this case. 


\subsubsection{Case of two nuclear phases at equilibrium}

If one allows for a finite external pressure $P_{0}$, one can always find (for $0<T<T_{\text {crit) }}$ ) two constant solutions of the Euler equation (6.50) which satisfy simultaneously the four equations

$$
\mathscr{F}_{\infty}^{\prime}\left(\rho_{0}\right)=\mathscr{F}_{\infty}^{\prime}\left(\rho_{\mathrm{g}}\right)=\lambda
$$

and

$$
P_{0}=\lambda \rho_{0}-\mathscr{F}_{\infty}\left(\rho_{0}\right)=P_{\mathrm{g}}=\lambda \rho_{\mathrm{g}}-\mathscr{F}_{\infty}\left(\rho_{\mathrm{g}}\right),
$$

the latter following from eq. (6.55). Equations (6.63, 6.64) can be numerically solved with the well-known Maxwell construction and yield uniquely $\rho_{0}, \rho_{\mathrm{g}}, \lambda$ and $P_{0}$ for a fixed temperature $T$. The solutions for $\rho_{0}, \rho_{\mathrm{g}}$ and $P_{0}$ are shown in fig. 15 by solid lines. In the pressure isotherms in fig. 16, the pairs of corresponding densities $\rho_{0}, \rho_{\mathrm{g}}$ and $P_{0}$ are indicated by dots connected by horizontal dashed lines. The critical temperature $T_{\text {crit }}$ at which $\rho_{0}$ and $\rho_{\mathrm{g}}$ become equal is $\sim 3 \mathrm{MeV}$ higher than that of the metastable situation $\left(T_{\text {crit }}^{\prime}\right)$ at $P_{0}=0$.

This phase equilibrium between condensed nuclear matter and a nucleon gas has been discussed extensively in the literature [101, 102] for various effective forces. As shown explicitly in ref. [101], the critical temperatures $T_{\text {crit }}$ and $T_{\text {crit }}^{\prime}$ become smaller with decreasing incompressibility $K_{\infty}$ of the force.

The gradient terms in eq. (6.50) determine the surface profile of $\rho$ which varies smoothly between $\rho_{0}$ and $\rho_{\mathrm{g}}$. Equation (6.50) was solved numerically in ref. [103] in the semi-infinite limit in the pure TF approximation (thus neglecting the term $\delta \mathscr{F}_{2}[\rho] / \delta \rho$ ) using a Skyrme force. An important point is that for any finite temperature $0<T<T_{\text {crit }}$, one finds solutions which approach exponentially the asymptotic values $\rho_{0}$ and $\rho_{\mathrm{g}}$ on either side. This result is not changed if the term $\delta \mathscr{F}_{2} / \delta \rho$ is taken into account. The ranges of the corresponding exponentials may be obtained similarly as in section 3.3. For the condensed region where $\rho \rightarrow \rho_{0}$, we can approximately use eqs. (3.26), (3.37) replacing $\rho_{\infty}$ by $\rho_{0}(T)$ and $K_{\infty}$ by the incompressibility $K_{0}$ of the hot, condensed phase, defined by $K_{0}(T)=9 \rho_{0} \mathrm{~d}^{2} \mathscr{F}_{\infty} /\left.\mathrm{d} \rho^{2}\right|_{\rho_{0}}$ * This corresponds to using the asymptotic values of $\zeta, \gamma$ and $\nu$ (eq. (6.45)) which, however, will not be exact for $T \geq 3 \mathrm{MeV}$ as seen from fig. 13. The asymptotic fall-off of $\rho(r)$ towards the gas density $\rho_{\mathrm{g}}$ can only be obtained analytically far outside (i.e. when $\tilde{\eta}$ becomes negative), where it is of little physical interest. In the true surface region, no simple form of the profile can be found due to the complicated $\rho$ dependence of the coefficients $\zeta$ and $\nu$ in eq. (6.53). We shall show in section 6.5, however, that parametrized trial densities lead to an excellent agreement with the exact surface energies found by numerical integration using eq. (6.55).

\subsection{Test of the TETF functionals against HF results}

We shall now present some tests of the TETF functionals eqs. (6.27-6.38) against HF calculations at finite temperatures (see also ref. [107]). In the kinetic energy density $\tau[\rho]$ and the spin-orbit density $J[\rho]$, we add hereby the fourth order terms valid for $T=0$ given in appendix A. We have used the Skyrme force SIII for which HF calculations at $T>0$ have been performed some time ago [99]. The calculations are limited to temperatures $T \leqslant 4 \mathrm{MeV}$ for which the effect of nucleon evaporation still can be neglected, i.e. $\rho_{\mathrm{g}}$ is smaller than $\sim 0.3 \times 10^{-3}$ so that it can be practically put equal to zero and we can

* The corresponding relation also holds in the case $P_{0}=0$ (free nucleus without external pressure). 


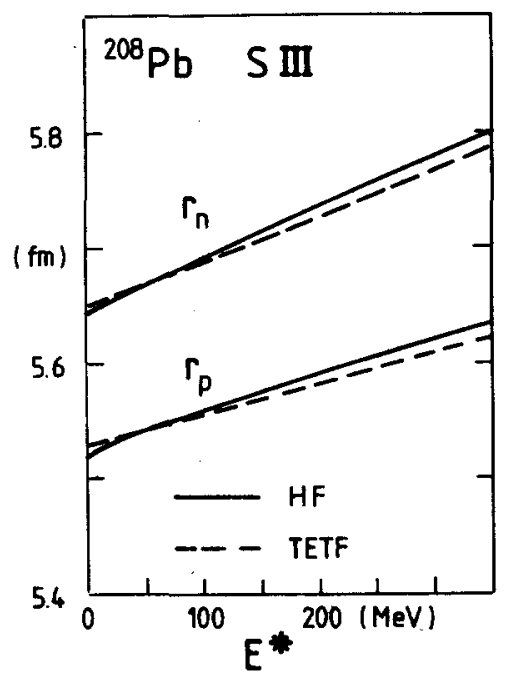

Fig. 17. R.m.s. radii of ${ }^{208} \mathrm{~Pb}$, calculated with the SIII force in the HF (solid lines) and the variational TETF approximation (dashed lines), plotted versus excitation energy $E^{*}$.

use the parametrization eq. (4.1) in the variational ETF calculations. (We also put $\rho_{1 \mathrm{q}}=0$; leaving it free would lead to a gain of less than $0.5 \mathrm{MeV}$ in the total binding energy for the force SIII; cf. section 4.2 and table 3).

In fig. 17 we compare the r.m.s. radii $r_{\mathrm{n}}$ and $r_{\mathrm{p}}$ obtained for ${ }^{208} \mathrm{~Pb}$ as functions of the intrinsic excitation energy $E^{*}$ defined by

$$
E^{*}=E(T)-E(0),
$$

where $E(T)$ is either the HF or the ETF energy at temperature $T$. An excellent agreement is reached; the relative difference between the two curves never exceeds $2.6 \times 10^{-3}$.

In fig. 18 we plot for the same nucleus the "effective level density parameter" $a_{\text {eff }}$ defined by

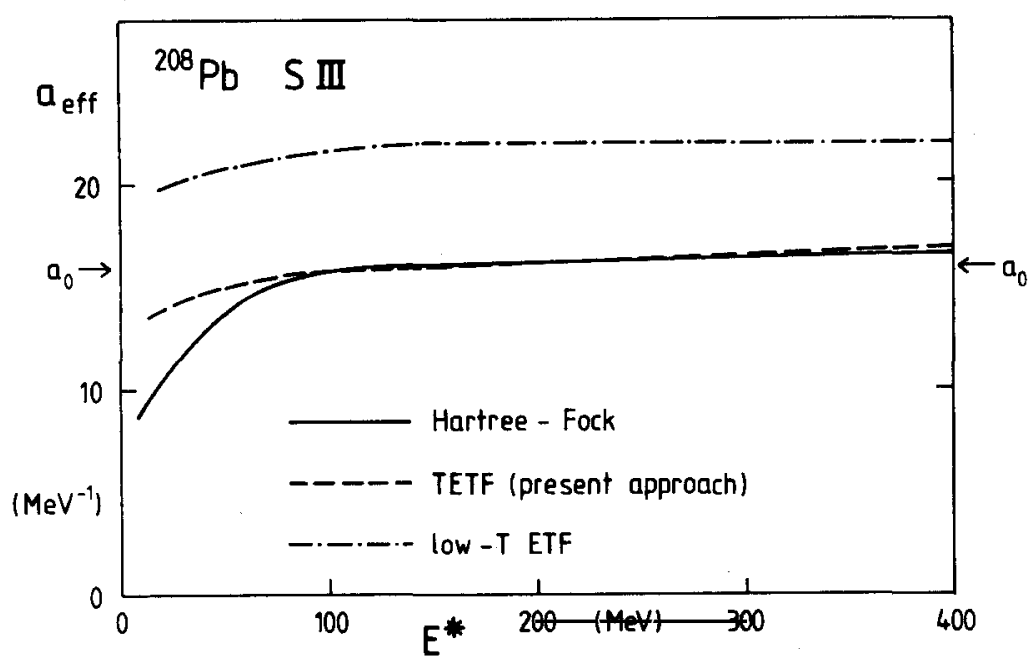

Fig. 18. Effective level density parameter $a_{\text {eff }}(6.66)$ of ${ }^{20} \mathrm{~Pb}$ with SIII force versus excitation energy $E^{*}$. Solid line: HF result [99]; dashed line: TETF result; dashed-dotted line: result obtained with the low-temperature expanded functional (6.6). 


$$
a_{\mathrm{eff}}=\frac{1}{4} \mathrm{~d} S^{2} / \mathrm{d} E^{*} \sim S / 2 T
$$

As already shown in the HF calculations of ref. [99], $a_{\text {eff }}$ approaches the parameter $a_{0}$ (eq. (6.8)) when the shell effects are washed out. Then, the relations of the "shifted Fermi gas model" [111] hold:

$$
\left.\begin{array}{l}
S \sim 2 \sqrt{a_{0}\left(E^{*}+\delta E\right)}, \\
E^{*} \sim a_{0} T^{2}-\delta E .
\end{array}\right\}
$$

Hereby $\delta E$ is the shell-correction energy of the ground state $(T=0$, see section 2.2$)$. We see that the semiclassical curve coincides perfectly with the $\mathrm{HF}$ curve above $E^{*} \approx 100 \mathrm{MeV}$ (corresponding to $T \simeq 2.5 \mathrm{MeV}$ ). Both quantities approach $a_{0}$ asymptotically; the slight deviation at large $E^{*}$ is due to the fact that $\tilde{g}(\lambda)$ and therefore $a_{0}$ (eq. (6.8)) is not constant as assumed in the Fermi gas theory (i.e. higher order terms in $T$ have to be added to eqs. (6.67) which become noticeable at $T \geqslant 4 \mathrm{MeV}$; see also the discussion in ref. [99]). We also show in fig. 18 the result obtained with the "low-temperature expansion" of the functional $\tau[\rho]$, eq. (6.6). It gives a drastic overestimation of $a_{\text {eff }}$ of more than $30 \%$. As already discussed in section 6.1.2, this is due to the inherent assumption $T \ll(\lambda-V)$ which is not justified in the nuclear surface. The bad result of the low-temperature expansion is therefore no surprise.

We have recently made a similar comparison using the functional eq. (6.4) in which the $T=0$ expression for $\tau_{2}[\rho]$ was used and $\sigma_{2}[\rho]$ was left out [105]. Although reasonable results were obtained, a discrepancy of $\sim 7 \%$ between the ETF and the HF results for $a_{\text {eff }}$ remained. This error can be traced back to the missing of the gradient correction $\sigma_{2}[\rho]$. Its contribution to the total entropy $S$ is found always to be negative (see eq. (6.34) and fig. 13 for $\nu(\tilde{\eta})$ ); it decreases in absolute value from $\sim 10$ at $T \simeq 1 \mathrm{MeV}$ to $\sim 8$ at $T \simeq 4 \mathrm{MeV}$. Its relative smallness (except at very low $T$ ) seems to justify our present neglection of fourth order corrections to $\sigma[\rho]$. (Model calculations for a harmonic oscillator potential indicate that $\sigma_{4}[\rho]$ is, indeed, negligible [112].)

As an illustration for the variation of the densities with temperature, we give in table 10 a list of their parameters (according to eq. (4.1) with $\rho_{1 \mathrm{q}}=0$ ), obtained for ${ }^{208} \mathrm{~Pb}$ with the realistic force $S k M^{*}$.

Table 10

Temperature dependence of r.m.s. radii (in $\mathrm{fm}$ ) and density variational parameters (asymmetric Fermi function profiles, units as table 3) obtained for ${ }^{2008} \mathrm{~Pb}$ with $\mathrm{SkM}^{*}$ force

\begin{tabular}{llllll}
\hline$T$ & 0 & 1 & 2 & 3 & $4(\mathrm{MeV})$ \\
\hline$r_{\mathrm{p}}$ & 5.46 & 5.47 & 5.50 & 5.54 & 5.61 \\
$r_{\mathrm{n}}$ & 5.62 & 5.63 & 5.67 & 5.74 & 5.86 \\
$R_{\mathrm{p}}$ & 6.98 & 6.95 & 6.90 & 6.84 & 6.77 \\
$R_{\mathrm{n}}$ & 7.19 & 7.14 & 7.04 & 6.86 & 6.61 \\
$\alpha_{\mathrm{p}}$ & 0.535 & 0.536 & 0.537 & 0.540 & 0.554 \\
$\alpha_{\mathrm{n}}$ & 0.658 & 0.654 & 0.644 & 0.627 & 0.589 \\
$\gamma_{\mathrm{p}}$ & 1.42 & 1.37 & 1.27 & 1.15 & 1.02 \\
$\gamma_{\mathrm{n}}$ & 1.56 & 1.47 & 1.30 & 1.06 & 0.79 \\
$\rho_{0 \mathrm{p}}$ & 0.0623 & 0.0623 & 0.0618 & 0.0609 & 0.0598 \\
$\rho_{0 \mathrm{~m}}$ & 0.0912 & 0.0910 & 0.0901 & 0.0884 & 0.0858 \\
\hline
\end{tabular}


We see that the central densities $\rho_{0 \mathrm{q}}$ slightly decrease with increasing temperature, which is also the case for the density of infinite nuclear matter. The most noticeable effect is that the asymmetry parameters $\gamma_{\mathrm{q}}$ clearly decrease. Thus the surface profiles become more symmetric when the temperature increases, which can be qualitatively understood by the smearing out of the Fermi surface causing a transport of nucleons from the inner parts of the nucleus to the outer surface. Note that the parameters practically do not change between $T=0$ and $T=1 \mathrm{MeV}$.

In summary, we have seen that the variational TETF method with trial nucleon densities is an excellent approximation* to the much more costly HF method; in particular at $T \geqslant 2.5 \mathrm{MeV}$ where the shell effects are washed out, a perfect agreement is obtained. This pertains also to the entropy which is a crucial quantity for an excited system.

The advantages of the semiclassical TETF method will be particularly gratifying in the calculation of the equation of state of hot nuclear matter with mixed gaseous and liquid (condensed) phases such as it plays an essential role in the collapse of massive stars [22]. The problem of correctly treating the continuum, which one meets in HF calculations [114], is completely circumvented in the variational TETF method where one deals only with local densities.

\subsection{Temperature dependence of fission barriers and LDM parameters}

A question which has been much discussed in the literature is how the fission barriers of excited nuclei depend on the temperature $[62,100,111]$. Microscopic HF calculations would be far too time consuming to study this problem systematically. As we have seen above, our TETF formalism gives a solid basis for doing this in an economical way.

As an illustration we want to discuss the semiclassical fission barrier obtained for the test nucleus ${ }^{240} \mathrm{Pu}$ using the Skyrme $\mathrm{SkM}^{*}$ force. To the extent that fission is an isothermal process (which is only approximately true), one has to look at the deformation behaviour of the free energy $F$. We calculated $F$ as in section 6.4 with densities $\rho_{\mathrm{q}}(r)$ going exponentially to zero at large distances.

Figure 19 shows the free deformation energy at temperatures from 0 to $4 \mathrm{MeV}$ versus the elongation parameter $c$ (along $h=0$ which closely follows the fission valley also at $T>0$ ). The Coulomb energy was calculated here in the liquid drop approximation. After adding the diffuseness correction $E_{c}^{\text {diff }}$ (see appendix D), we obtain the barrier heights (i.e. the heights of the saddle points) shown in fig. 20.

The decrease of the barrier heights with increasing temperature is mainly due to a decrease of the surface free energy [101,115] (see below). Up to $T \simeq 2.5 \mathrm{MeV}$ one has to add the shell-correction to these results to obtain a complete picture. In this region, the average barrier does not decrease very much; the main part of the excitation energy thus goes into "melting" the shell effects. Once these have disappeared at $T \geqslant 2.5 \mathrm{MeV}$, our results become quantitative and completely equivalent to those of a HF calculation; the barrier then is reduced rather quickly and has practically disappeared at $T \simeq$ $4 \mathrm{MeV}$. Note that the low-temperature expansion, which has been recently used to predict the temperature dependence of fission barriers [62], gives a completely wrong result: due to the overestimation of the level density parameter $a_{\text {eff }}$ noticed in fig. 17 above, it leads also to a drastic overestimation of the temperature effect on the barrier heights.

As a final application, we present the LDM parameters obtained in the leptodermous expansion of the free energy $F$ of a heated nuclear system. The procedure is exactly the same as described in section 5 , except that one starts from the total free energy $F$ (or the Gibbs potential $G=E-T S+P_{0} V$, if a

* This clearly contradicts recent claims based on pure TF calculations [113] (see also the results at the end of this section, table 11). 


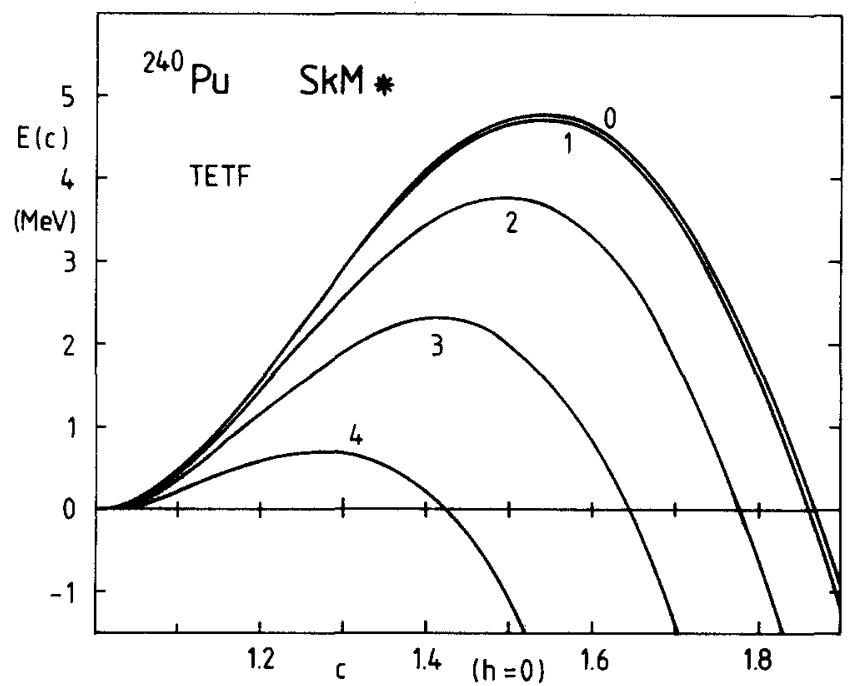

Fig. 19. Fission barriers of ${ }^{240} \mathrm{Pu}$ (as in fig. 4) obtained with the $\mathrm{SkM}^{*}$ force at various temperatures (given by the numbers in $\mathrm{MeV}$ ) using the variational TETF approach. The Coulomb diffuseness correction (see appendix D) is not contained here.

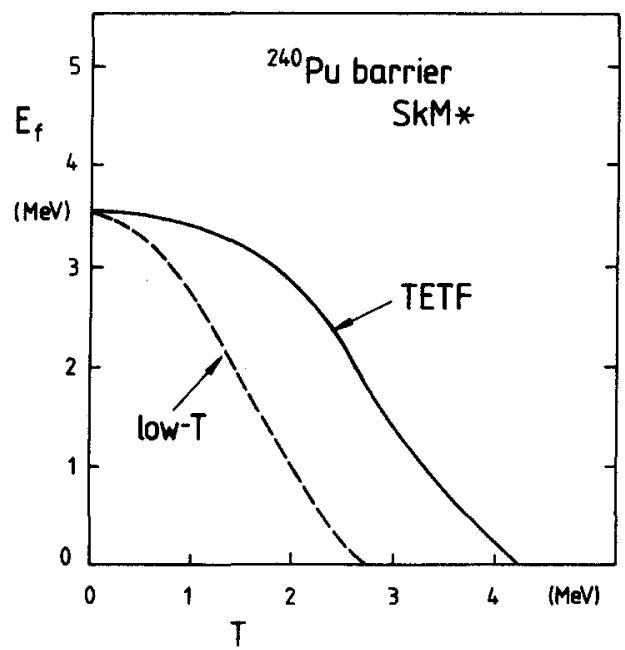

Fig. 20. Barrier beights from fig. 19, after adding the Coulomb diffuseness correction, versus temperature. The corresponding results obtained with the low- $T$ expanded functional (6.6) is shown by the dashed curve.

nonzero external pressure $P_{0}$ is present, as discussed in section 6.3.2). In terms of the density profile $\rho(z)$ of semi-infinite nuclear matter, the surface (free) energy is then given by (see also ref. [103])

$$
a_{\mathrm{s}}=4 \pi r_{0}^{2} \int_{-\infty}^{+\infty}\left\{\mathscr{F}[\rho]-\lambda \rho(z)+P_{0}\right\} \mathrm{d} z
$$

where $r_{0}=\left(4 \pi \rho_{0} / 3\right)^{-1 / 3}$ and $\rho_{0}(T)$ is the density of the condensed nuclear matter phase. In our variational calculations, we have used the TETF functionals described in section 6.2 and again added 
the $T=0$ expressions of $\tau_{4}[\rho]$ (see appendix A). The density profile $\rho(z)$ was parametrized (for symmetric nuclear matter) as

$$
\rho(z)=\rho_{\mathrm{g}}+\left(\rho_{0}-\rho_{\mathrm{g}}\right) /[1+\exp (z / \alpha)]^{\gamma}
$$

here $\rho_{\mathrm{g}}$ is the nucleon gas density discussed in section 6.3. The parameters $\alpha$ and $\gamma$ were determined for each temperature by minimizing $a_{\mathrm{s}}$ (eq. (6.68)). The curvature energy coefficient $a_{\mathrm{c}}^{\infty}$ and the constant parameter $a_{0}^{\infty}$ are then obtained as discussed in section 5 in terms of the first and second moments of the integrand in eq. (6.68).

The results are shown in fig. 21 as functions of the temperature for the two choices of $P_{0}$ discussed in section 6.3. The curvature energy $a_{c}$ also includes the compression energy and corresponds to the quantity $a_{\mathrm{c}}^{*}$ in eq. (5.31). All LDM parameters are seen to go to zero at the critical temperatures where $\rho_{0}$ and $\rho_{\mathrm{g}}$ become equal and the profile $\rho(z)$ is a constant.

The dashed curves correspond to the situation of a free, metastable nucleus without external pressure $\left(P_{0}=0\right)$. As we have seen in section 6.3.1, the profile $\rho(z)$ should go to $\rho_{\mathrm{g}}$ with zero slope at a finite point $z_{0}$. The parametrization (6.69) forces this point to infinity; we do not believe, however, that this has an important effect on the results. In any case, these results with $P_{0}=0$ are only of a schematic nature. In a real finite nucleus, the asymmetry $(N \neq Z)$ and in particular, the Coulomb interaction will tend to lower appreciably the critical temperature $T_{\text {crit }}^{\prime}$.

The solid curves in fig. 21 correspond to an equilibrium situation such as it is assumed in calculations of the equation of state of hot nuclear matter in the context of stellar collapse. In LDM type calculations it was recently shown [116] that the curvature energy $a_{\mathrm{c}}$ plays a sensitive role for the occurrence of the so-called bubble phase [22]. It is therefore important to know also its correct temperature dependence.

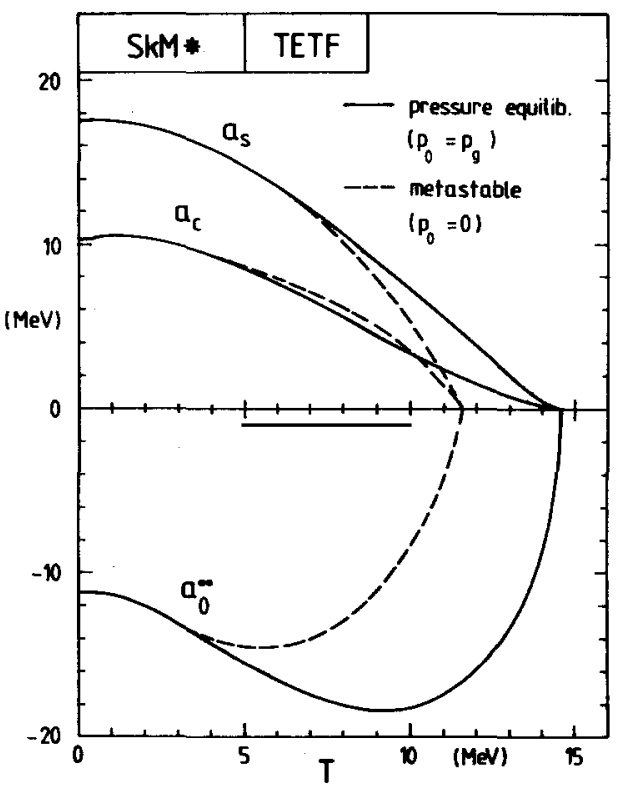

Fig. 21. LDM parameters obtained from symmetric semi-infinite nuclear matter calculations with the variational TETF method using the SkM* force, plotted versus temperature. The solid curves correspond to the equilibrium situation, the dashed curves to that of a metastable isolated nucleus without external pressure. (Cf. fig. 15 for the corresponding limiting densities $\rho_{0}$ and $\rho_{\mathrm{k}}$.) 
Similar calculations for the surface energy $a_{\mathrm{s}}$ were also performed in refs. $[103,117]$ in the pure TF approximation for the equilibrium situation $\left(P_{0}=P_{\mathrm{g}}\right)$. In ref. [117], the metastable situation $\left(P_{0}=0\right)$ was also considered and it was observed that the results for $a_{\mathrm{s}}$ in both situations agree up to $T \simeq 7 \mathrm{MeV}$. Our results in fig. 21 confirm this although, of course, the absolute values of $a_{\mathrm{s}}$ are changed by the inclusion of the semiclassical corrections to the density functionals. For the coefficients $a_{c}$ and $a_{0}^{\infty}$, however, the two curves start deviating from each other already at lower temperatures. Up to $T \simeq 3 \mathrm{MeV}$, the results for all coefficients agree in the two situations. We can conclude therefore that for a finite nucleus the presence of an external pressure does not change the total free energy up to $T \simeq 3-4 \mathrm{MeV}$. In fact, the outside density $\rho_{\mathrm{g}}$ can be put equal to zero up to these temperatures without affecting too much any of the observables. This justifies our treatment of finite nuclei using $\rho_{\mathrm{g}}=0$ in section 6.4 and above in the present section. This result is also in accordance with the confirmation of the old HF results [99] up to about $T \simeq 3 \mathrm{MeV}$ by recent $\mathrm{HF}$ calculations in which the continuum states were treated more carefully [114].

In order to test the quality of the parametrization eq. (6.69) of the density profile $\rho(z)$, we have calculated the surface energy $a_{\mathrm{s}}$ exactly, using only the second order gradient terms in the TETF functionals (thus ignoring $\tau_{4}[\rho]$ ). In this case, the Euler variational equation can be integrated once (see section 6.3). From eq. (6.55) it follows then that the surface energy also can be written as*

$$
a_{\mathrm{s}}=4 \pi r_{0}^{2} \cdot 2 \int_{-\infty}^{+\infty}\left\{\mathscr{F}_{\infty}(\rho)-\lambda \rho(z)+P_{0}\right\} \mathrm{d} z
$$

where $\mathscr{F}_{\infty}(\rho)$ is given in eq. (6.38). This means that exactly one half of the surface energy comes from the "bulk" terms and the other half from the second order gradient corrections (including the term $b(\nabla \rho)^{2}$ in the potential energy). This result is quite general and can be derived from scale invariance in the same way as one can derive the virial theorem. (If fourth order terms are included, a similar "theorem" can be found with different factors in front of the zeroth, second and fourth order gradient terms, respectively [112].)

With the substitution $\mathrm{d} z=\mathrm{d} \rho / \rho^{\prime}(z)$ and using once more eq. (6.55), the integral in eq. (6.70) can be transformed into

$$
a_{\mathrm{s}}=8 \pi r_{0}^{2} \int_{\rho_{\mathrm{g}}}^{\rho_{0}} \sqrt{b+\frac{\hbar^{2}}{2 m} \frac{1}{\rho} \zeta(\tilde{\eta})} \cdot \sqrt{\mathscr{F}_{\infty}(\rho)-\lambda \rho+P_{0}} \mathrm{~d} \rho
$$

which is a simple one-dimensional integral over known functions of $\rho$. We have integrated eq. (6.71) numerically for the equilibrium case $\left(P_{0}=P_{\mathrm{g}}\right)$ using the force $\mathrm{SkM}^{*}$. The results are given in table 11 in the second column $\left(a_{\mathrm{s}}^{\mathrm{ex}}\right)$ for various temperatures. The third and fourth columns contain the variational results using the parametrization (6.69), varying either both parameters $\alpha$ and $\gamma$ (giving $a_{s}^{\gamma \neq 1}$ ), or only the parameter $\alpha$, keeping $\gamma=1$ (giving $a_{\mathrm{s}}^{\gamma=1}$ ). The agreement between $a_{\mathrm{s}}^{\text {ex }}$ and $a_{\mathrm{s}}^{\gamma \neq 1}$ is striking; the relative difference never exceeds $0.25 \%$. The error made by keeping symmetric profiles with $\gamma=1$ is largest at $T=0$ (where the optimal $\gamma_{0}$ is found to be $\simeq 9$ ) and quite small at $T=10 \mathrm{MeV}$ (where

* In the case $P_{0}=0$, the upper integration limit should, strictly, be replaced by $z_{0}$; see fig. 14 in section 6.3 . 
Table 11

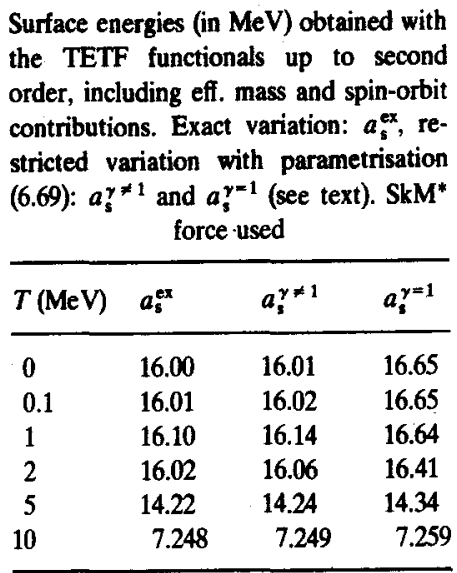

$\gamma_{0} \simeq 1.3$ ). The value $\gamma_{0} \simeq 9$ at $T=0$ is unrealistic and due to the missing fourth order gradient terms in $\tau[\rho]$ (see the discussion in section 3.3).

We conclude from this test that the parametrization of $\rho(z)$ (eq. (6.69)) is indeed sufficient to reproduce the exact surface energy following from the Euler equation with a high accuracy. Inclusion of the fourth order terms $\tau_{4}[\rho]$, which tend to make the profiles more symmetric (i.e. $\gamma$ closer to 1 ), should not make the situation less favourable.*

\section{Summary}

We have reviewed the basic justifications for the use of semiclassical density functionals in the calculation of static average nuclear properties. We have in particular demonstrated the necessity of including the fourth order gradient corrections to the ETF functionals $\tau[\rho]$ and $J[\rho]$ in order to obtain both accurate total energies and realistic surfaces of the nucleon densities.

In semiclassical variational calculations using the ETF density functionals and Skyrme type effective interactions, we obtain quantitative agreement with the results of averaged HF calculations for nuclear densities, binding and deformation energies. After perturbative inclusion of the shell effects by a single HF iteration, the exact total HF energies are reproduced within less than $2 \times 10^{-3}$ for heavy nuclei. In particular for the calculation of fission barriers, the semiclassical method is shown to be extremely efficient. In this way, for the first time a Skyrme force-labeled SkM* - has been determined which leads both to excellent binding energies, densities and radii of stable spherical nuclei and to a realistic fission barrier of a typical actinide nucleus.

With the help of a "leptodermous" expansion of the ETF binding energy and with calculations for semi-infinite nuclear matter, we establish a link between Skyrme type forces and liquid drop or droplet models. For a series of forces we give all the droplet model parameters. We also have discussed some shortcomings of the droplet model and proposed some extensions and improvements.

Finally, we have discussed the recent extension of the ETF model to finite temperatures. We have shown that the low-temperature expanded functionals give poor results whereas the new TETF functionals, including the exact temperature dependence up to the second order gradients, lead to a

* See ref. [112] for a corresponding analysis including the fourth order gradient terms. 
perfect agreement with HF results at temperatures $T \geqslant 2.5 \mathrm{MeV}$ where the shell effects are washed out. We have also discussed the important new result that taking the proper limit $T \rightarrow 0$ from the finite-temperature theory, the ETF functionals $\tau[\rho]$ and $J[\rho]$ at $T=0$ are strictly valid also in the classically forbidden region. As further illustrations, we have studied the temperature dependence of fission barriers and of LDM parameters pertinent to astrophysical applications.

\section{Appendix A: Fourth order gradient corrections to the ETF functionals}

\section{A1. Local case}

For the sake of completeness, we repeat here the explicit form of $\tau_{4}[\rho]$ for a local potential $[15,56]$ :

$$
\begin{aligned}
\tau_{4}[\rho]= & \frac{1}{4320}\left(3 \pi^{2}\right)^{-2 / 3} \rho^{1 / 3}\left[24 \frac{\Delta^{2} \rho}{\rho}-60 \frac{\nabla \rho \cdot \nabla(\Delta \rho)}{\rho^{2}}\right. \\
& \left.-28\left(\frac{\Delta \rho}{\rho}\right)^{2}-14 \frac{\Delta(\nabla \rho)^{2}}{\rho^{2}}+\frac{280}{3} \frac{(\nabla \rho)^{2} \Delta \rho}{\rho^{3}}+\frac{184}{3} \frac{\nabla \rho \cdot \nabla(\nabla \rho)^{2}}{\rho^{3}}-96\left(\frac{\nabla \rho}{\rho}\right)^{4}\right]
\end{aligned}
$$

The functional derivative of $\tau_{4}[\rho]$ which appears in the Euler equation (3.23) is

$$
\begin{aligned}
D_{4}[\rho]=\frac{\delta \tau_{4}}{\delta \rho}= & \left(3 \pi^{2}\right)^{-2 / 3} \rho^{-2 / 3} \frac{1}{6480}\left[-176\left(\frac{\nabla \rho}{\rho}\right)^{4}+\frac{544}{3}\left(\frac{\Delta \rho}{\rho}\right)\left(\frac{\nabla \rho}{\rho}\right)^{2}\right. \\
& \left.+112 \frac{\nabla \rho \cdot \nabla(\nabla \rho)^{2}}{\rho^{3}}-66\left(\frac{\Delta \rho}{\rho}\right)^{2}-106 \frac{\nabla \rho \cdot \nabla(\Delta \rho)}{\rho^{2}}-27 \frac{\Delta(\nabla \rho)^{2}}{\rho^{2}}+48 \frac{\Delta^{2} \rho}{\rho}\right]
\end{aligned}
$$

\section{A2. Nonlocal case}

The complete functionals $\tau_{4}[\rho]$ and $J_{4}[\rho]$ for a nonlocal Hamiltonian of Skyrme type eq. (3.19) have been given in ref. [16]. In the present context we only need the integral of the total Skyrme energy density eq. (2.5). After suitable partial integrations, many terms cancel and only up to second derivatives of the density $\rho(r)$, the spin-orbit potential $W(r)$ and the effective mass $m^{*}(r)$ (for each kind of nucleons) remain under the integral. In the total Skyrme energy, $\tau_{q}(r)$ and $J_{q}(r)$ only occur in the following combinations [see eq. (2.5)]

$$
\sum_{\mathbf{q}} f_{\mathbf{q}}(\boldsymbol{r}) \tau_{\mathbf{q}}(\boldsymbol{r}), \quad \sum_{\mathbf{q}} \boldsymbol{J}_{\mathbf{q}}(\boldsymbol{r}) \cdot \boldsymbol{W}_{\mathbf{q}}(\boldsymbol{r}) \quad(\mathrm{q}=\mathrm{n}, \mathrm{p})
$$

where the fields $f_{\mathrm{q}}(\boldsymbol{r})=m / m_{\mathrm{q}}^{*}(\boldsymbol{r})$ and $\boldsymbol{W}_{\mathrm{q}}(\boldsymbol{r})$ are given by eqs. (2.10) and (2.11), respectively. It is thus sufficient to perform the partial integrations for the products $f \tau[\rho]$ and $W \cdot J[\rho]$ for one kind of particles once for all.

The result for $\int f \tau_{4}[\rho]$ is, without spin-orbit contributions: 


$$
\begin{aligned}
\int f \tau_{4}[\rho] \mathrm{d}^{3} r= & \left(3 \pi^{2}\right)^{-2 / 3} \int \mathrm{d}^{3} r \rho^{1 / 3}\left\{\frac{1}{270} f\left(\frac{\Delta \rho}{\rho}\right)^{2}-\frac{1}{240} f \frac{\Delta \rho}{\rho}\left(\frac{\nabla \rho}{\rho}\right)^{2}+\frac{1}{810} f\left(\frac{\nabla \rho}{\rho}\right)^{4}-\frac{1}{240} \frac{(\Delta f)^{2}}{f}\right. \\
& +\frac{1}{120} \frac{\Delta f(\nabla f)^{2}}{f^{2}}-\frac{1}{240} \frac{(\nabla f)^{4}}{f^{3}}+\frac{1}{360} \Delta f \frac{(\nabla f \cdot \nabla \rho)}{f \rho}-\frac{1}{360} \Delta \rho \frac{(\nabla f)^{2}}{f \rho}-\frac{7}{2160} \Delta f\left(\frac{\nabla \rho}{\rho}\right)^{2} \\
& +\frac{1}{540}\left(\frac{\nabla \rho}{\rho}\right)^{2} \frac{(\nabla f)^{2}}{f}+\frac{7}{2160} \frac{(\nabla \rho \cdot \nabla f)^{2}}{f \tilde{\rho}^{2}}-\frac{11}{3240}\left(\frac{\nabla \rho}{\rho}\right)^{2} \frac{(\nabla \rho \cdot \nabla f)}{\rho} \\
& \left.+\frac{7}{1080} \Delta \rho \frac{(\nabla \rho \cdot \nabla f)}{\rho^{2}}+\frac{1}{180} \Delta f \frac{\Delta \rho}{\rho}\right\} .
\end{aligned}
$$

The spin-orbit potential $\boldsymbol{W}_{\mathrm{q}}(\boldsymbol{r})$ enters at two places: once in the spin-orbit energy proper [i.e., the second term in eq. (A.3)] and once through the functional $\tau[\rho]$ [see also the expression (3.20) for the second order term]. Since the resulting contributions to the two kinds of terms in eq. (A.3) have the same form and partially cancel, it is practical to combine them, which yields (for one kind of nucleons)

$$
\begin{aligned}
\int\left[\frac{h^{2}}{2 m} f \tau_{4}^{\mathrm{w}}[\rho]+W \cdot J_{4}[\rho]\right] \mathrm{d}^{3} r= & \left(3 \pi^{2}\right)^{-2 / 3}\left(\frac{m}{\hbar^{2}}\right) \int f^{-1} \rho^{1 / 3}\left\{\frac{1}{4}(\nabla \cdot W)^{2}-\frac{3}{8}(\nabla \cdot W) \frac{(W \cdot \nabla f)}{f}\right. \\
& +\frac{1}{16}(W)^{2} \frac{\Delta f}{f}+\frac{1}{8} \frac{(W \cdot \nabla f)^{2}}{f^{2}}+\frac{1}{24}(\nabla \cdot W) \frac{(W \cdot \nabla \rho)}{\rho}+\frac{1}{48}(W)^{2} \frac{\Delta \rho}{\rho} \\
& \left.-\frac{1}{72}(W)^{2}\left(\frac{\nabla \rho}{\rho}\right)^{2}+\frac{1}{2}\left(\frac{m}{\hbar^{2}}\right)^{2} \frac{(W)^{4}}{f^{2}}\right\} \mathrm{d}^{3} r
\end{aligned}
$$

Note that in $\tau_{4}^{\varpi}[\rho]$, only those contributions are contained which explicitly include the spin-orbit potential $W$; all the other contributions are contained in eq. (A.4).

\section{Appendix B: Expectation values of two-body operators in the ETF approach}

We shall briefly sketch the calculation of expectation values of two-body operators within the ETF model. Let $\hat{O}^{(2)}$ be a two-body operator which in the HF ground state has the following expectation value

$$
\left\langle\hat{O}^{(2)}\right\rangle=\sum_{i, j}^{N}\left\langle i j\left|\hat{O}^{(2)}\right| \overline{i j}\right\rangle=\sum_{i, j}^{N}\left\langle i j\left|\hat{O}^{(2)}\left(1-\hat{P}_{\mathrm{r}} \hat{P}_{\sigma}\right)\right| i j\right\rangle
$$

Here $\hat{P}_{\sigma}=\left(1+\sigma_{1} \cdot \sigma_{2}\right) / 2$ is the spin-exchange operator and $\hat{P}_{\mathrm{r}}$ the spatial (Majorana) exchange operator. (We assume that $\hat{O}^{(2)}$ does not mix neutron and proton states and consider only one kind of nucleons.) The ETF model is consistent with the assumption of plane waves for the spatial part of the wavefunctions, so that the spinors decouple and, for any system with time reversal symmetry, the sums over the spin matrix elements $\left\langle i j\left|\sigma_{1} \cdot \sigma_{2}\right| i j\right\rangle$ vanish. For a spin-independent operator $\hat{O}\left(r_{1}, r_{2}\right)$, the expectation value (B.1) can then be written as 


$$
\begin{aligned}
\left\langle\hat{O}^{(2)}\right\rangle= & \int \mathrm{d}^{3} r_{1} \int \mathrm{d}^{3} r_{2} \int \mathrm{d}^{3} r_{1}^{\prime} \int \mathrm{d}^{3} r_{2}^{\prime} \delta\left(r_{1}-r_{1}^{\prime}\right) \delta\left(r_{2}-r_{2}^{\prime}\right) \hat{O}\left(r_{1}, r_{2}\right) \\
& \times\left[\rho\left(r_{1}, r_{1}^{\prime}\right) \rho\left(r_{2}, r_{2}^{\prime}\right)-\frac{1}{2} \rho\left(r_{2}, r_{1}^{\prime}\right) \rho\left(r_{1}, r_{2}^{\prime}\right)\right]
\end{aligned}
$$

where the factor $1 / 2$ in the exchange term comes from the operator $\hat{P}_{\sigma}$, and $\rho\left(r_{1}, r_{2}\right)$ is the spatial density matrix

$$
\rho\left(\boldsymbol{r}_{1}, \boldsymbol{r}_{2}\right)=\sum_{i=1}^{N} \varphi_{i}^{*}\left(\boldsymbol{r}_{2}\right) \varphi_{i}\left(\boldsymbol{r}_{1}\right) .
$$

The ETF approximation to $\rho\left(r_{1}, r_{2}\right)$ is obtained by the inverse Laplace transform eq. (3.3) of the Wigner-Kirkwood expansion of the nonlocal Bloch density $C\left(\boldsymbol{r}_{1}, \boldsymbol{r}_{2} ; \beta\right)$. The latter is most easily expressed in terms of centre-of-mass and relative coordinates

$$
R=\frac{1}{2}\left(r_{1}+r_{2}\right), \quad s=r_{1}-r_{2} .
$$

For a local potential $V(r)$, it has the following form [118]:

$$
\begin{aligned}
C(\boldsymbol{R}, s ; \beta)= & \frac{1}{4}\left(\frac{2 m}{\pi \beta \hbar^{2}}\right)^{3 / 2} \exp \{-\beta V(\boldsymbol{R})\} \exp \left\{-\frac{m}{2 \hbar^{2} \beta} s^{2}\right\} \\
& \times\left\{1+\frac{\hbar^{2}}{24 m}\left[-2 \beta^{2} \Delta_{R} V(\boldsymbol{R})+\beta^{3}\left[\nabla_{R} V(\boldsymbol{R})\right]^{2}-\beta \frac{m}{\hbar^{2}}\left(s \cdot \nabla_{R}\right)^{2} V(\boldsymbol{R})\right]+\cdots\right\} .
\end{aligned}
$$

To lowest order in $\hbar$, one obtains the familiar Slater density matrix

$$
\rho_{\mathrm{TF}}(\boldsymbol{R}, s)=\rho_{\mathrm{TF}}(\boldsymbol{R}) \frac{3}{s k_{\mathrm{F}}} j_{1}\left(s k_{\mathrm{F}}\right),
$$

where

$$
k_{\mathrm{F}}(\boldsymbol{R})=\left[3 \pi^{2} \rho_{\mathrm{TF}}(\boldsymbol{R})\right]^{1 / 3}, \quad s=|s| .
$$

The higher order terms in eq. (B.5) lead to Bessel functions of higher order, similarly as in the quantum-mechanical density matrix expansion (DME) [119]. (For a discussion of the DME in the ETF approach, see ref. [118].)

As an example, consider the c.m. correction to the HF energy

$$
E_{\mathrm{cm}}=-\left\langle\boldsymbol{P}^{2}\right\rangle / 2 M,
$$

where $M=N m$ is the total mass and $P=\Sigma_{i} p_{i}$ is the total momentum, $E_{\mathrm{cm}}$ thus having a one-body and a two-body part:

$$
E_{\mathrm{cm}}=E_{\mathrm{cm}}^{(1)}+E_{\mathrm{cm}}^{(2)}=-\frac{1}{2 M}\left\{\sum_{i=1}^{N}\left\langle i\left|p^{2}\right| i\right\rangle+\sum_{i j}^{N}\left\langle i j\left|p_{1} \cdot p_{2}\right| \widehat{i j}\right\rangle\right\} .
$$


The one-body part gives just the total kinetic energy which we can write as

$$
E_{\mathrm{cm}}^{(1)}=-\frac{\hbar^{2}}{2 M} \int \mathrm{d}^{3} r \tau(r)
$$

In the two-body part, the direct matrix elements are all zero, so that only the exchange part remains. With the above expression (B.2) we get

$$
\begin{aligned}
E_{\mathrm{cm}}^{(2)} & =\frac{1}{2 M} \sum_{i j}^{N}\left\langle i j\left|p_{1} \cdot p_{2}\right| j i\right\rangle \\
& =-\frac{\hbar^{2}}{2 M} \frac{1}{2} \int \mathrm{d}^{3} R \int \mathrm{d}^{3} s\left\{\frac{1}{4}\left[\nabla_{R} \rho(R, s)\right]^{2}-\left[\nabla_{s} \rho(R, s)\right]^{2}\right\}
\end{aligned}
$$

To order TF [neglecting all gradients of $\rho(\boldsymbol{R})$ ] one finds

$$
E_{\mathrm{cm}}^{(2)}=\frac{\hbar^{2}}{2 M} \frac{1}{2} \int \mathrm{d}^{3} R \rho_{\mathrm{TF}}^{2}(R) \int \mathrm{d}^{3} s\left\{\frac{\mathrm{d}}{\mathrm{d} s}\left[\frac{3}{s k_{\mathrm{F}}} j_{1}\left(s k_{\mathrm{F}}\right)\right]\right\}^{2} .
$$

After evaluating the integration over $s$, one finds

$$
E_{\mathrm{cm}}^{(2)}=\frac{\hbar^{2}}{2 m} \int \mathrm{d}^{3} r \tau_{\mathrm{TF}}(r)
$$

Thus the two-body term cancels exactly the one-body term (B.10) at TF level. Keeping all gradients of $\rho$ up to second order and all terms of order $\hbar^{2}$ in eq. (B.5), one gets various terms with integrals over products of Bessel functions, some of which diverge. Keeping only the finite terms, one obtains the same result as eq. (B.13) but including also $\tau_{2}[\rho]$, so that the cancellation takes place also at the level of the second order contributions. The physically relevant part of the c.m. correction (B.9) thus lies in the diverging terms and is not accessible in the ETF approximation to the density matrix $\rho\left(r_{1}, r_{2}\right)$.

\section{Appendix C: Intrinsic curvilinear coordinate system and leptodermous expansion of moments of the density}

In view of their repeated applications in this paper, we shall briefly recall here the main formulae for the curvilinear "intrinsic" coordinate system which was originally introduced in ref. [64] and used for Skyrme-ETF calculations in ref. [86].

We start from an axially symmetric "generating" liquid drop type surface with sharp edges, which in the usual cylindrical coordinates $(\rho, z, \varphi)$ is given by

$$
\rho=f(z) \text {. }
$$

The curvilinear coordinate system $(u, v, \varphi)$ is found as illustrated in fig. 22: from any point $\mathbf{P}$ in the 


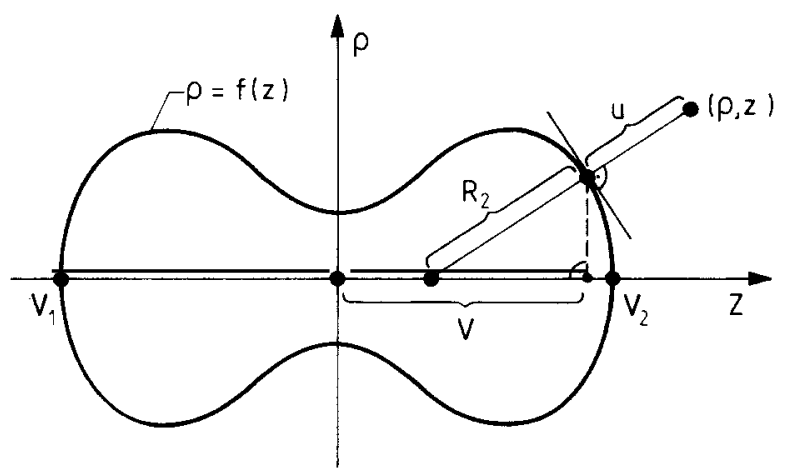

Fig. 22. Connection between curvilinear coordinates $(u, v)$ and usual cylindrical coordinates $(\rho, z)$ for an axially symmetric deformed nucleus. The shape function $p=f(z)$ defines the sharp "generating" surface.

$(\rho, z)$-plane, one constructs the normal onto the sharp surface. The $z$-coordinate of the projection of $\mathrm{P}$ on the surface is the new coordinate $v$; the distance of $\mathbf{P}$ from the surface along the normal is the new coordinate $u$ (taken to be positive outside and negative inside the sharp surface). The relation between these coordinates is

$$
\begin{aligned}
& z=v-u f^{\prime}(v) / \sigma(v) \\
& \rho=f(v)+u / \sigma(v)
\end{aligned}
$$

with

$$
\sigma(v)=+\sqrt{1+\left[f^{\prime}(v)\right]^{2}}
$$

The system $(u, v, \varphi)$ is orthogonal; the volume element is

$$
\mathrm{d}^{3} r=\rho \mathrm{d} \rho \mathrm{d} z \mathrm{~d} \varphi=g(u, v) \mathrm{d} u \mathrm{~d} v \mathrm{~d} \varphi
$$

with the Jacobian

$$
g(u, v)=\left(1+u / R_{1}\right)\left(u+R_{2}\right) .
$$

Here we have used the principle curvature radii $R_{1}, R_{2}$ which are given in terms of $f(v)$ (eq. (C.1)) and $\sigma(v)$ (eq. (C.3)) by

$$
R_{1}^{-1}=-f^{\prime \prime}(v) /[\sigma(v)]^{3}, \quad R_{2}=\sigma(v) f(v) .
$$

The ranges of the variables are $v_{1} \leq v \leq v_{2}$ and $-R_{2} \leq u<+\infty$. The volume $V$ inside the sharp surface defined by eq. (C.1) (i.e. by $u=0$ ) is

$$
V=2 \pi \int_{v_{1}}^{v_{2}} \mathrm{~d} v \int_{-R_{2}}^{0} g(u, v) \mathrm{d} u=\pi \int_{v_{1}}^{v_{2}} f^{2}(v) \mathrm{d} v
$$


the surface area is given by

$$
S=2 \pi \int_{v_{1}}^{v_{2}} f(v) \sigma(v) \mathrm{d} v=2 \pi \int_{v_{1}}^{v_{2}} R_{2} \mathrm{~d} v=\oint \mathrm{d} S .
$$

Below we also need integrals over the mean curvature $H$ and the Gaussian curvature $G$ :

$$
H=\frac{1}{2}\left(R_{1}^{-1}+R_{2}^{-1}\right), \quad G=\left(R_{1} R_{2}\right)^{-1}
$$

The surface integral over $H$, which we in the following just call the "mean curvature" $C$, is

$$
C=2 \pi \int_{v_{1}}^{v_{2}} H R_{2} \mathrm{~d} v=\pi \int_{v_{1}}^{v_{2}}\left(1+R_{2} / R_{1}\right) \mathrm{d} v=\oint \mathrm{d} C .
$$

The surface integral over $G$ is constant:

$$
2 \pi \int_{v_{1}}^{v_{2}} G R_{2} \mathrm{~d} v=2 \pi \int_{v_{1}}^{v_{2}} \frac{1}{R_{1}} \mathrm{~d} v=4 \pi
$$

as a special case of the Gauss-Bonné law. (For disconnected shapes consisting of $n$ separate pieces, the integral in eq. (C.11) will give $n \cdot 4 \pi$. This is easily proved by substituting $x=f^{\prime}(v)$ and integrating over $x$.)

The approximation to the semiclassical nuclear densities used in this paper is that they only depend on the variable $u$ (see section 4.3). In this case, the square of the gradient and the Laplacian of $\rho$ are given by

$$
\begin{aligned}
& |\nabla \rho(u)|^{2}=(\mathrm{d} \rho / \mathrm{d} u)^{2}=\left[\rho^{\prime}(u)\right]^{2}, \\
& \Delta \rho(u)=\rho^{\prime \prime}(u)+\rho^{\prime}(u)\left[\frac{1}{u+R_{1}}+\frac{1}{u+R_{2}}\right]
\end{aligned}
$$

We shall now derive the so-called "leptodermous expansion" of the particle number for a general type of density profile which is flat inside the nucleus, with central value $\rho_{0}$, and drops to zero in a relatively narrow region around the surface, i.e. within $-\alpha \leq u \leq+\alpha$, so that $\alpha$ is always much smaller than the curvature radius $\boldsymbol{R}_{2}$ :

$$
\alpha \ll R_{2} \text {. }
$$

Introducing the dimensionless variable $x=u / \alpha$, we write

$$
\begin{array}{ll}
\rho(u)=\rho_{0} & y(u / \alpha)=\rho_{0} y(x) ; \\
y(x) \rightarrow 0 & (x \gg 1), \\
y(x) \rightarrow 1 & (x \ll-1) .
\end{array}
$$


The profile of the sharp density is

$$
\rho^{(0)}(u)=\rho_{0} \Theta(-u)
$$

We now write the total particle number $A$ as the sum of the number of particles contained in the sharp volume $V$ and the rest:

$$
\begin{aligned}
& A=\int \mathrm{d}^{3} r \rho(u)=\rho_{0} V+\Delta A, \\
& \Delta A=\rho_{0} 2 \pi \int_{v_{1}}^{v_{2}} \mathrm{~d} v \int_{-R_{2}}^{\infty} g(u, v) \delta \rho(u) \mathrm{d} u,
\end{aligned}
$$

where

$$
\delta \rho(u)=\rho(u)-\rho^{(0)}(u)=\rho_{0}[y(u / \alpha)-\theta(-u)]
$$

By construction, $\delta \rho(u)$ and thus the integrand of the second part in eq. (C.17) is peaked around $u=0$. In the leptodermous approximation eq. (C.14), we can therefore replace the lower limit of the $u$-integration in $\Delta A$ by $-\infty$ without introducing much of an error. Using the explicit form of the Jacobian $g(u, v)$ (eq. (C.5)), the integrals in $\Delta A$ then separate and we obtain

$$
\begin{aligned}
\Delta A & \approx 2 \pi \rho_{0} \alpha \int_{v_{1}}^{v_{2}} \mathrm{~d} v \int_{-\infty}^{+\infty} \mathrm{d} x R_{2}\left[1+2 H \alpha x+G \alpha^{2} x^{2}\right][y(x)-\Theta(-x)] \\
& =\rho_{0} \alpha\left(c_{0} S+2 \alpha c_{1} C+4 \pi \alpha^{2} c_{2}\right)
\end{aligned}
$$

whereby eqs. (C.8-C.11) have been used, and we have defined

$$
c_{n}=\int_{0}^{\infty} x^{n}\left[y(x)+(-1)^{n} y(-x)+(-1)^{n+1}\right] \mathrm{d} x \quad(n=0,1,2)
$$

The error made in the approximation (C.19) contains integer powers of the small number $\exp \left(-R_{2} / \alpha\right)$, so that we can write for the particle number

$$
A=\rho_{0}\left\{V+c_{0} \alpha S+2 c_{1} \alpha^{2} C+4 \pi \alpha^{3} c_{2}\right\}+\mathcal{O}\left(\exp \left(-R_{2} / \alpha\right)\right)
$$

Note that the coefficients $c_{n}$ depend only on the form of the profile function $y(x)$ and not on the deformation of the system. In case of a profile which is symmetric around $x=u=0$ (e.g. for a Fermi function), one will have $c_{0}=c_{2}=0$. For a spherical system one may introduce a "sharp radius" $R_{0}\left(=R_{1}=R_{2}\right)$; then

$$
V=\frac{4}{3} \pi R_{0}^{3}, \quad S=4 \pi R_{0}^{2}, \quad C=4 \pi R_{0},
$$


and $A$ (eq. (C.21)) becomes essentially a finite series in powers of $\left(\alpha / R_{0}\right)$, which can be inverted to give

$$
R_{0}=r_{0} A^{1 / 3}-c_{0} \alpha+\left(c_{0}^{2}-2 c_{1}\right) \frac{\alpha^{2}}{r_{0}} A^{-1 / 3}+O\left(A^{-2 / 3}\right)
$$

with $r_{0}=\left(4 \pi \rho_{0} / 3\right)^{-1 / 3}$.

Similarly we obtain for the mean square radius of the density profile $\rho(u)$ (eq. (C.15))

$$
\begin{aligned}
\left\langle r^{2}\right\rangle & =\frac{1}{A} \int \mathrm{d}^{3} r r^{2} \rho(u) \\
& =\frac{1}{A} \frac{4 \pi}{5} \rho_{0} R_{0}^{5}\left[1+5 c_{0}\left(\frac{\alpha}{R_{0}}\right)+20 c_{1}\left(\frac{\alpha}{R_{0}}\right)^{2}+30 c_{2}\left(\frac{\alpha}{R_{0}}\right)^{3}+\cdots\right] .
\end{aligned}
$$

Making use of eq. (C.21) we can write this as

$$
\left\langle r^{2}\right\rangle=\frac{3}{5} R_{0}^{2}\left[1+2 c_{0}\left(\alpha / R_{0}\right)+\left(14 c_{1}+9 c_{0}^{2}\right)\left(\alpha / R_{0}\right)^{2}+\cdots\right] .
$$

For Fermi function profiles $\rho(u)$, the value of $c_{1}$ is $\pi^{2} / 6$ and $c_{0}=c_{2}=0$. An alternative expression for the coefficients $c_{n}$ (eq. (C.20)) is

$$
c_{n}=\frac{-1}{(n+1)} \int_{-\infty}^{+\infty} x^{n+1} \frac{\mathrm{d} y(x)}{\mathrm{d} x} \mathrm{~d} x \quad(n=0,1,2)
$$

\section{Appendix D: Leptodermous expansion of the Coulomb energy}

We shall discuss here the lowest terms of the leptodermous expansion of the classical Coulomb energy for a charge distribution $e \rho(r)$

$$
E_{\mathrm{c}}=\frac{e^{2}}{2} \int \mathrm{d}^{3} r \int \mathrm{d}^{3} r^{\prime} \frac{\rho(\boldsymbol{r}) \rho\left(\boldsymbol{r}^{\prime}\right)}{|\bar{r}-\boldsymbol{r}|}
$$

(Here $\rho(r)$ is the density of the $Z$ protons; we omit for simplicity the index p.) We shall assume again that $\rho(r)$ only depends on the distance $u$ from a generating sharp surface (see eq. (C.15)). We also introduce an "equivalent sharp density" $\rho^{(\varepsilon)}(u)$ by

$$
\rho^{(\varepsilon)}(u)=\rho_{0} \Theta\left(\frac{\varepsilon-u}{\alpha}\right)
$$

where $\varepsilon$ is at the moment an arbitrary distance which shall be fixed later. Writing

$$
\rho(u)=\rho^{(\varepsilon)}(u)+\delta \rho(u)
$$


we expand as in ref. [19] the energy $E_{c}$ around $\rho^{(\varepsilon)}$ to get

$$
E_{\mathrm{c}}=E_{\mathrm{LD}}^{(\varepsilon)}+E^{(1)}+E^{(2)}
$$

where $E_{\mathrm{Lb}}^{(\varepsilon)}$ is the usual liquid drop model Coulomb energy of the equivalent sharp density which can readily be computed (see e.g. refs. $[19,37])$. The correction $E^{(1)}$ of first order in $\delta \rho$ can be written as

$$
E^{(1)}=e \int \mathrm{d}^{3} r V^{(\varepsilon)}(\boldsymbol{r}) \delta \rho(u)
$$

here $V^{(\varepsilon)}$ is the Coulomb potential corresponding to the distribution $\rho^{(\varepsilon)}(u)$ (eq. (D.2)). The idea now is to use a leptodermous expansion of $E^{(1)}$ and to keep only its lowest terms. The term $E^{(2)}$ in eq. (D.4) is of second order in $\delta \rho$ and hoped to be practically negligible. This shall be tested numerically below.

Myers and Swiatecki [19] derived a "theorem" telling that the leading term in $E^{(1)}$, which is of order $\alpha^{2}$ relative to $E_{\mathrm{LD}}^{(\varepsilon)}$, is independent of the deformation of the density distribution $\rho(r)$. We shall show in the following that it is not exactly true. Let us first rewrite eq. (D.5) in the curvilinear coordinate system described in appendix $\mathrm{C}$ above:

$$
E^{(1)}=2 \pi e \int_{v_{1}}^{v_{2}} \mathrm{~d} v R_{2} \int_{-\infty}^{+\infty} \mathrm{d} u\left[1+2 u H+u^{2} G\right] V^{(\varepsilon)}(u, v) \delta \rho(u)
$$

where we have used the leptodermous limit $\alpha \ll R_{2}$ to replace the lower limit of the $u$-integration by $-\infty$. The next step is, similarly to ref. [19], to expand $V^{(\varepsilon)}(u, v)$ around the equivalent sharp surface $u=\varepsilon$ :

$$
E^{(1)}=e \int_{-\infty}^{+\infty} \mathrm{d} u \delta \rho(u)[\oint \mathrm{d} S+2 u \oint \mathrm{d} C+\cdots]\left[V^{(\varepsilon)}(u=\varepsilon, v)+(u-\varepsilon) \frac{\mathrm{d} V^{(\varepsilon)}}{\mathrm{d} u}(u=\varepsilon, v)+\cdots\right] .
$$

The integrals over the surface $S$ and the curvature $C$ are defined in eqs. (C.8, 10). Collecting only those terms in eq. (D.7) which will be of order $\alpha$ and $\alpha^{2}$, we obtain

$$
\begin{aligned}
E^{(1)}= & e \int_{-\infty}^{+\infty} u \mathrm{~d} u \delta \rho(u) \oint \mathrm{d} S \frac{\mathrm{d} V^{(\varepsilon)}}{\mathrm{d} u}(u=\varepsilon, v) \\
& +e \int_{-\infty}^{+\infty} \mathrm{d} u \delta \rho(u)[\oint \mathrm{d} S+2 u \oint \mathrm{d} C] V^{(\varepsilon)}(u=\varepsilon, v)+\mathcal{O}\left(\alpha^{3}\right)
\end{aligned}
$$

The first term in eq. (D.8) can be partially integrated using the Gauss law into

$$
E_{1}^{(1)}=-4 \pi e^{2} Z(\varepsilon) \int_{-\infty}^{+\infty} u \delta \rho(u) \mathrm{d} u
$$


where $Z(\varepsilon)$ is the number of protons contained in the equivalent sharp volume of $\rho^{(\varepsilon)}$ (eq. (D.2)). In ref. [19], $\varepsilon$ was chosen such that $Z(\varepsilon)=Z$, and the second and third terms of eq. (D.8) were neglected. One then could write

$$
E_{\mathrm{MS}}^{(1)}=-4 \pi e^{2} Z \int_{-\infty}^{+\infty} u \mathrm{~d} u \delta \rho(u)
$$

which, indeed, is independent of the deformation of the charge distribution and has been used throughout in the droplet model as the "diffuseness correction" to the Coulomb energy (see the coefficient $c_{3}$ in ref. [19]). The neglection of the second term of eq. (8) was argued saying that $\int_{-\infty}^{+\infty} \delta \rho(u) \mathrm{d} u=0$; this is, however, incorrect. (The neglection of the third term was not discussed at all.)

From eqs. (D.2) and (C.20) we obtain

$$
\begin{aligned}
& \int_{-\infty}^{+\infty} \delta \rho(u) \mathrm{d} u=\rho_{0}\left(c_{0} \alpha-\varepsilon\right), \\
& \int_{-\infty}^{+\infty} u \delta \rho(u) \mathrm{d} u=\rho_{0}\left(c_{1} \alpha^{2}-\frac{1}{2} \varepsilon^{2}\right) .
\end{aligned}
$$

If $\varepsilon$ is chosen to give $Z(\varepsilon)=Z$, as in ref. [19], then the integral eq. (D.11) will differ from zero, as can be seen from eq. (C.21), and of the same order as the other terms in eq. (D.8). For a spherical distribution $\rho(r)$ we get with eq. (C.23)

$$
\varepsilon=c_{0} \alpha-\left(c_{0}^{2}-2 c_{1}\right) \alpha^{2} / R_{0}+O\left(\alpha^{3}\right)
$$

and thus

$$
\begin{aligned}
& \int_{-\infty}^{+\infty} \delta \rho(u) \mathrm{d} u=\rho_{0}\left(c_{0}^{2}-2 c_{1}\right) \alpha^{2} / R_{0}+\mathcal{O}\left(\alpha^{3}\right) \\
& \int_{-\infty}^{+\infty} u \delta \rho(u) \mathrm{d} u=\rho_{0}\left(c_{1}-\frac{1}{2} c_{0}^{2}\right) \alpha^{2}+\mathcal{O}\left(\alpha^{3}\right)=-\frac{R_{0}}{2} \int_{-\infty}^{+\infty} \delta \rho(u) \mathrm{d} u+O\left(\alpha^{3}\right)
\end{aligned}
$$

Also, for the spherical case we know the analytical form of $V^{(\varepsilon)}$ at the surface $u=\varepsilon$ :

$$
V_{0}^{(\varepsilon)}(u=\varepsilon)=Z e / R_{\varepsilon}=Z e / R_{0}+\mathcal{O}(\alpha)
$$

with 


$$
R_{\varepsilon}=R_{0}+\varepsilon
$$

For any deformed distribution, we define the surface and curvature integrals over $V^{(e)}(u=\varepsilon, v)$ as

$$
\begin{aligned}
& \oint \mathrm{d} S V^{(\varepsilon)}(u=\varepsilon, v)=4 \pi R_{0} V_{0}^{(\varepsilon)} B_{\mathrm{cs}} \\
& \oint \mathrm{d} C V^{(\varepsilon)}(u=\varepsilon, v)=4 \pi R_{0}^{2} V_{0}^{(\varepsilon)} B_{\mathrm{cc}}
\end{aligned}
$$

so that $B_{\mathrm{cs}}=B_{\mathrm{cc}}=1$ in the spherical case. With this, we obtain for the exact correction $E^{(1)}$ up to order $\alpha^{2}$ :

$$
E^{(1)}=-4 \pi Z e^{2}\left[1-2\left(B_{\mathrm{cc}}-B_{\mathrm{cs}}\right)\right] \int_{-\infty}^{+\infty} u \delta \rho(u) \mathrm{d} u+\mathscr{O}\left(\alpha^{3}\right)
$$

Comparing to eq. (D.10) we note that the two terms neglected by Myers and Swiatecki [19] exactly cancel at sphericity, but each of them is twice as large as the term $E_{\mathrm{MS}}^{(1)}$ which was retained. It must therefore be tested numerically how they behave for deformed shapes.

In fig. 23 we show the sum of the two extra terms, i.e. the quantity

$$
\Delta E^{(1)}=4 \pi Z e^{2} \cdot 2\left(B_{\mathrm{cc}}-B_{\mathrm{cs}}\right) \int_{-\infty}^{+\infty} u \delta \rho(u) \mathrm{d} u
$$

for a realistic case, namely ${ }^{240} \mathrm{Pu}$ along the approximate fission path (see section 4.3 for the definition of

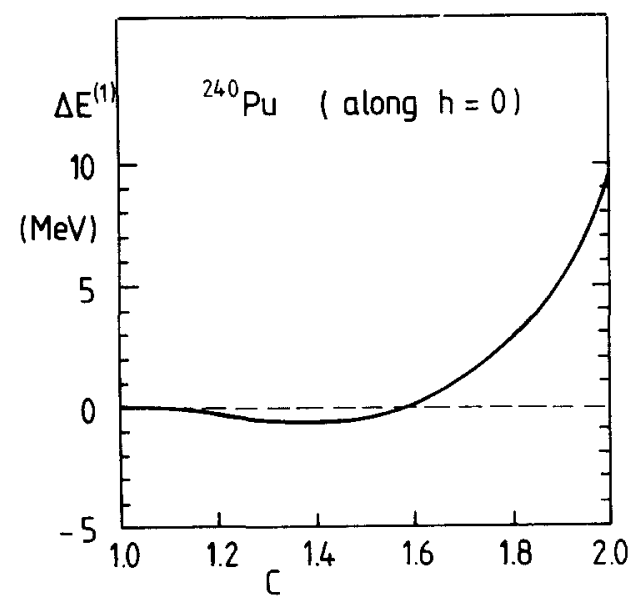

Fig. 23. Correction $\Delta E^{(1)}$ (eq. (D.21)) to the Coulomb energy "theorem" of Myers and Swiatecki [19] discussed in the text, versus elongation $c$ for the nucleus ${ }^{240} \mathrm{Pu}$. 
the deformation parameters). The constant term $E_{\mathrm{M}}^{(1)}$ (eq. (D.10)) is in this case equal to $-39 \mathrm{MeV}$, i.e. the two extra terms are of the order of $\sim 80 \mathrm{MeV}$ each. We see that they cancel also up to deformations somewhat beyond the saddle point to within $<1 \mathrm{MeV}$; beyond $c \approx 1.6 \mathrm{MeV}$ they add up to a larger positive error. We can conclude that - at least for the application to the fission barriers of heavy nuclei discussed in this paper - the "theorem" of Myers and Swiatecki holds within less than $\sim 1 \mathrm{MeV}$ for the relevant part of the deformation energy curve, but it should be noted that this is due to a cancellation of two individually large contributions which they neglected.

The second order term $E^{(2)}$ in eq. (D.4) cannot be expanded in the same easy way. We studied it numerically by calculating the exact total Coulomb energy $E_{\mathrm{c}}$ (eq. (D.1)) and subtracting from it the constant part $E_{\mathrm{MS}}^{(1)}$ (eq. (D.10)) of the first order contribution. The difference

$$
\Delta E_{\mathrm{c}}^{\mathrm{diff}}=E_{\mathrm{c}}-E_{\mathrm{MS}}^{(1)}
$$

contains thus the sum of $\Delta E^{(1)}$ (eq. (D.21)), all terms of order $\alpha^{3}$ and higher, and $E^{(2)}$. It is shown in fig. 24 for the same test case ${ }^{240} \mathrm{Pu}$ along the fission path and seen to be negative in the whole region of interest; outside the saddle point (indicated by an arrow) it reaches a minimum of $\sim-1.4 \mathrm{MeV}$. The curve in fig. 24 is typical for all Skyrme forces studied in this paper; the curves obtained for other parametrisations than $\mathrm{SkM}^{*}$ agree with it to less than $\sim 0.2 \mathrm{MeV}$. To the same accuracy it is also independent of the parameter $\gamma$. We find thus that another cancellation takes place between $\Delta E^{(1)}$ seen in fig. 23 at larger deformations and $E^{(2)}$ (and possibly the terms of order $\alpha^{3}$ in $E^{(1)}$ ).

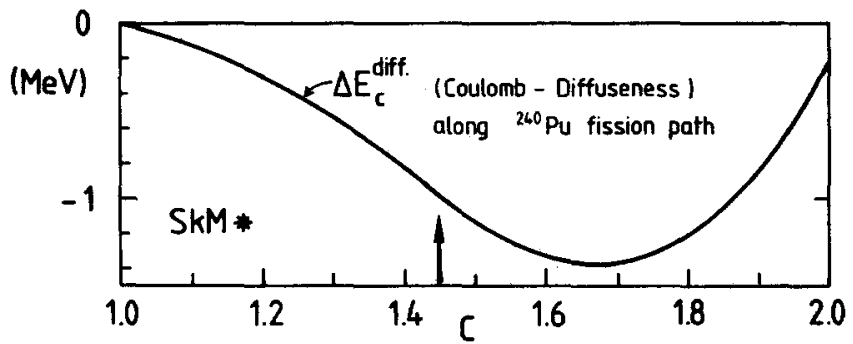

Fig. 24. Total diffuseness correction $\Delta E_{c}^{\text {dif }}$ (eq. (D.22)) to the Coulomb energy of ${ }^{200} \mathrm{Pu}$ versus elongation $c$. The curve is approximately the same (within $\sim 0.2 \mathrm{MeV}$ ) for different realistic forces and independent of the asymmetry $\left(\gamma_{\mathrm{p}}\right)$ of the proton density profile.

The final conclusion is that a total error of less than $\sim 1.5 \mathrm{MeV}$ is made along the semiclassical fission path of actinides, if the exact Coulomb energy is replaced by the LDM part $E_{\mathrm{LB}}^{(\varepsilon)}$ plus the constant part $E_{\mathrm{Ms}}^{(1)}$ (eq. (D.10)) of the leptodermous correction; hereby the equivalent sharp density $\rho^{(\varepsilon)}(u)$ is that which contains the correct number $Z$ of protons. This result is, of course, based on the use of densities with the form eq. (4.1) (with $\rho_{\mathbf{q} 1}=0$ ) obtained variationally for the various forces.

\section{Acknowledgements}

We have benefitted from many stimulating discussions with M. Asghar, J. Bartel, R. Bengtsson, R.K. Bhaduri, M. Durand, H. Gräf, B. Grammaticos, R.W. Hasse, B.K. Jennings, H. Krivine, J. Meyer, H. Nifenecker, J.R. Nix, J.M. Pearson, P. Quentin, P. Schuck, C. Signarbieux, W. Stocker, F. Tondeur, J. Treiner and A. Voros. One of us (C.G.) acknowledges the hospitality of the University of Regensburg 
and one (M.B.) that of the Centre d'Etudes Nucléaires at Grenoble. We are grateful to J. Bartel, M. Farine and $\mathrm{W}$. Stocker for a critical reading of the first draft of this article, which was typed by Mme. Petit in a singular effort within a few days. Mrs. G. Korinth and Mrs. A. Herrmann were heroic in correcting many pages over and over; Mrs. C. Geiger made most of the drawings. To all of them our sincere thanks.

\section{References}

[1] P. Quentin and H. Flocard, Ann. Rev. Nucl. Part. Sci. 28 (1978) 523.

[2] T.H.R. Skyrme, Phil. Mag. 1 (1956) 1043; Nucl. Phys. 9 (1959) 615.

[3] D. Vautherin and D.M. Brink, Phys. Rev. C5 (1972) 626.

[4] H. Flocard, P. Quentin, D. Vautherin, D. Veneroni and A.K. Kerman, Nucl. Phys. A231 (1974) 176.

[5] M. Brack, in: Physics and Chemistry of Fission 1979, Jülich (IAEA Vienna, 1980) Vol. I, p. 227.

[6] R.K. Bhaduri and C.K. Ross, Phys. Rev. Lett. 27 (1971) 606.

[7] J. Bartel, P. Quentin, M. Brack, C. Guet and H.B. Hàkansson, Nucl. Phys. A386 (1982) 79.

[8] C.F. v. Weizsäcker, Z. Phys. 96 (1935) 431.

[9] H.A. Bethe and F. Bacher, Rev. Mod. Phys. 8 (1936) 82.

[10] R.A. Berg and L. Wilets, Phys. Rev. 101 (1956) 201;

L. Wilets, Rev. Mod. Phys. 30 (1958) 542.

[11] K. Kumar and R.K. Bhaduri, Phys. Rev. 122 (1961) 1926.

[12] K.A. Brückner, J.R. Buchler, S. Jorna and R.J. Lombard, Phys. Rev. 171 (1968) 1188; H.A. Bethe, Phys. Rev. 167 (1968) 879.

[13] R.J. Lombard, Ann. of Phys. 77 (1973) 380.

[14] J.P. Hohenberg and W. Kohn, Phys. Rev. 136 (1964) B864.

[15] M. Brack, B.K. Jennings and Y.H. Chu, Phys. Lett. 65B (1976) 1.

[16] B. Grammaticos and A. Voros, Ann. of Phys. 123 (1979) 359; 129 (1980) 153.

[17] M. Brack and P. Quentin, Nucl. Phys. A361 (1981) 35.

[18] M. Brack, Habilitationsschrift, University of Frankfurt/M, 1977, unpublished (ILL preprint 77 BR 346 S).

[19] W.D. Myers and W.J. Swiatecki, Ann. of Phys. 55 (1969) 395; 84 (1974) 186.

[20] V.M. Strutinsky, Nucl. Phys. A95 (1967) 420; A122 (1968) 1.

[21] M. Lefort, Nucl. Phys. A387 (1982) 3c, and references quoted therein.

[22] J.M. Lattimer, Ann. Rev. Nucl. Part. Sci. 31 (1981) 337, and references quoted therein.

[23] P. Bonche and D. Vautherin, Nucl. Phys. A372 (1981) 496; Astron. Astrophys. 112 (1982) 168.

[24] M.J. Giannoni and P. Quentin, Phys. Rev. C21 (1980) 2076.

[25] D. Vautherin, Phys. Rev. C7 (1973) 296.

[26] C. Titin-Schnaider and P. Quentin, Phys. Lett. 49B (1974) 397.

[27] F. Tondeur, Nucl. Phys. A315 (1978) 353.

[28] O. Bohigas, A.M. Lane and J. Martorell, Phys. Reports 51 (1979) 267.

[29] J.P. Blaizot, Phys. Reports 64 (1980) 171.

[30] M. Beiner, H. Flocard, N.V. Giai and P. Quentin, Nucl. Phys. A238 (1975) 29.

[31] J. Treiner, H. Krivine, O. Bohigas and J. Martorell, Nucl. Phys. A371 (1981) 253.

[32] F. Tondeur, 4th Conf. on nuclei far from stability, Helsingor 1981 (CERN 81-09, Geneva) p. 81.

[33] S. Köhler, Nucl. Phys. A258 (1976) 301.

[34] H. Krivine, J. Treiner and O. Bohigas, Nuel. Phys. A366 (1980) 155.

[35] R. Balian and C. Bloch, Ann. of Phys. 69 (1972), and earlier references quoted therein.

[36] V.M. Strutinsky, A.G. Magner, S.R. Ofengenden and T. Dessing, Z. Phys. A283 (1977) 269.

[37] M. Brack, J. Damgård, A.S. Jensen, H.C. Pauli, V.M. Strutinsky and C.Y. Wong, Rev. Mod. Phys. 44 (1972) 320.

[38] M. Brack and H.C. Pauli, Nucl. Phys. A207 (1973) 401.

[39] A.S. Tyapin, Sov. J. Nucl. Phys. 11 (1970) 401; 14 (1972) 50.

[40] D.A. Kirzhnits, Sovj. Phys. JETP 5 (1957) 64; Field Theoretical Methods in Many Body Systems (Pergamon, Oxford, 1967).

[41] C.H. Hodges, Can. J. Phys. 51 (1973) 1428.

[42] E.P. Wigner, Phys. Rev. 40 (1932) 749.

[43] J.G. Kirkwood, Phys. Rev. 44 (1933) 31.

[44] B.K. Jennings, Nucl. Phys. A207 (1973) 538.

[45] B.K. Jennings, R.K. Bhaduri and M. Brack, Nucl. Phys. A253 (1975) 29, and references quoted therein. 
[46] M. Levy and J. Perdew, Int. J. Quantum Chem. 21 (1982) 511, and references quoted therein.

[47] G.E. Uhlenbeck and E. Beth, Physica 3 (1936) 729.

[48] B.K. Jennings, Ph.D. Thesis, McMaster University, 1976 (unpublished).

[49] A. Voros, Thèse d'Etat, Paris University, Orsay, 1977 (unpublished).

[50] R.K. Bhaduri, Phys. Rev. Lett. 39 (1977) 329.

[51] M. Durand, M. Brack and P. Schuck, Z. Phys. A286 (1978) 381; A29 (1980) 87.

[52] J. Bartel, M. Durand and M. Brack, Z. Phys. A315 (1984) 341.

[53] J. Bartel and M. Vallieres, Phys. Lett. 114B (1982) 303.

[54] J. Bartel, Ph.D. thesis, University of Regensburg 1984, and to be published.

[55] N.L. Balazs and B.K. Jennings, Phys. Reports 104 (1984) 347.

[56] C. Guet and M. Brack, Z. Phys. A297 (1980) 247.

[57] W. Stocker, G. Süssmann and S. Knaak, Nucl. Phys. A187 (1972) 38.

[58] O. Bohigas, X. Campi, H. Krivine and J. Treiner, Phys. Lett. 64B (1976) 381.

[59] H. Krivine and J. Treiner, Phys. Lett. 88B (1979) 212.

[60] X. Campi and S. Stringari, Nucl. Phys. A337 (1980) 313.

[61] J. Treiner and H. Krivine, Preprint Orsay IPNO/TH 82-18, 1982.

[62] M. Barranco, M. Pi and X. Viñas, Phys. Lett. 124B (1983) 131; X. Campi and S. Stringari, Z. Phys. A309 (1983) 239.

[63] E. Lieb, Rev. Mod. Phys. 53 (1981) 603.

[64] V.M. Strutinsky and A.S. Tyapin, Sov. Phys. JETP 18 (1964) 664.

[65] Y.H. Chu, B.K. Jennings and M. Brack, Phys. Lett. 68B (1977) 40; see also Y.H. Chu, Ph.D. Thesis, Stony Brook, 1977 (unpublished).

[66] J. Damgaard, H.C. Pauli, V.V. Pashkevitch and V.M. Strutinsky, Nucl. Phys. A135 (1969) 432.

[67] H.C. Pauli, Phys. Reports 7 (1973) 35.

[68] C. Guet, R. Bengtsson and M. Brack, as in ref. [5], Vol. II, p. 411.

[69] C. Guet, H.B. Hakkansson and M. Brack, Phys. Lett. 97B (1980) 7.

[70] J.B. Bellicard et al., Saclay progress report CEA-N-2207, 1981, p. 81.

[71] J.L. Friar and J.W. Negele, Adv. Nucl. Phys. 8 (1976) 219.

[72] F. Tondeur, M. Brack, M. Farine and J.M. Pearson, Nucl. Phys. A420 (1984) 297.

[73] Ph. Quentin, These d'Etat, Orsay, 1975 (unpublished).

[74] C.M. Ko, H.C. Pauli, M. Brack and G.E. Brown, Nucl. Phys. A236 (1974) 269.

[75] C.W. Hoffmann et al., Phys. Rev. C21 (1980) 1488; L. Ray, Nucl. Phys. A335 (1980) 443.

[76] A. Chaumeaux, V. Layly and R. Schaeffer, Ann. of Phys. 116 (1978) 247.

[77] G.D. Althazov et al., Nucl. Phys. A381 (1982) 430.

[78] I. Brissaud et al., Nucl. Phys. A191 (1972) 157.

[79] J.F. Berger and M. Girod, as in ref. [5], Vol. I, p. 264.

[80] J.F. Berger, M. Girod and D. Gogny, J. Physique Lett. 42 (1981) L-509.

[81] D. Gogny, Nucl. Phys. A237 (1975) 399.

[82] W.D. Myers and W.J. Swiatecki, Nucl. Phys. 81 (1966) 1.

[83] V.M. Strutinsky, N. Lyashchenko and N.A. Popov, Nucl. Phys. 46 (1963) 639.

[84] M. Brack, Phys. Lett. 81B (1977) 239.

[85] M. Brack, P. Quentin and D. Vautherin, Int. Symp. on Superheavy Elements, ed. M. Lodhi (Pergamon, New York, 1978 ) p. 309.

[86] M. Brack, C. Guet, H.-B. Hakansson, A. Magner and V.M. Strutinsky, 4th Int. Conf. on Nuclei Far From Stability, Helsingor, 1981 (CERN 81-09, Geneva) p. 65.

[87] W. Stocker, Nucl. Phys. A215 (1973) 591.

[88] D. Berdichevsky and U. Mosel, Nucl. Phys. A388 (1982) 205.

[89] M. Brack and W. Stocker, Nucl. Phys. A388 (1982) 230.

[90] J.M. Pearson, M. Farine and J. Côté, Phys. Rev. C26 (1982), and references quoted therein; M. Farine, Ph.D. Thesis, Montreal, 1981 (unpublished).

[91] W. Stocker and M. Farine, Ann. Phys. 159 (1985) 255.

[92] B. Grammaticos, Z. Phys. A312 (1983) 99.

[93] R.G. Seyler and C.H. Blanchard, Phys. Rev. 124 (1961); 131 (1963) 355.

[94] See various authors in Atom. Data and Nucl. Data Tables 17 (1976) 428.

[95] P. Müller and J.R. Nix, Nucl. Phys. A361 (1981) 117.

[96] M. Brack and W. Stocker, Nucl. Phys. A406 (1983) 413.

[97] M. Pearson, Nucl. Phys. A376 (1982) 507.

[98] F. Tondeur, J.M. Pearson and M. Farine, Nucl. Phys. A394 (1983) 462.

[99] M. Brack and P. Quentin, Phys. Lett. 52B (1974) 159; Physica Scripta A10 (1974) 163; see also ref. [17]. 
[100] U. Mosel, P. Zint and K.H. Passler, Nucl. Phys. A236 (1974) 252;

G. Sauer, H. Chandra and U. Mosel, Nucl. Phys. A264 (1976) 221.

[101] W. Stocker and J. Burzlaff, Nucl. Phys. A202 (1973) 265.

[102] W.A. Küpper, G. Wegmann and E. Hilf, Ann. Phys. 88 (1974) 454;

M. Barranco and J.R. Buchler, Phys. Rev. C22 (1980) 1729;

D.Q. Lamb et al., Nucl. Phys. A360 (1981) 459.

[103] D.G. Ravenhall, C.J. Pethick and J.M. Lattimer, Nucl. Phys. A407 (1983) 571.

[104] M. Barranco and J. Treiner, Nucl. Phys. A351 (1981) 269.

[105] J. Bartel, M. Brack, C. Guet and H.-B. Håkansson, Phys. Lett. 139B (1984) 1.

[106] M. Brack, in: Workshop on Semiclassical Methods in Nuclear Physics, Grenoble, 1984: J. Phys. (Paris) Colloques C6 (1984) 15.

[107] M. Brack, Phys. Rev. Lett. 53 (1984) 119.

[108] E.S. Stoner, Phil. Mag. 28 (1939) 257.

[109] M. Brack, Phys. Rev. Lett. 54 (1985) 851.

[110] See, e.g., H.N.V. Temperley, Proc. Phys. Soc. 59 (1947) 199.

[111] P.B. Kahn and N. Rosenzweig, Phys. Rev. 187 (1970) 1193;

V.S. Ramamurthy, S.S. Kapoor and S.K. Kataria, Phys. Rev. C5 (1972) 1124.

[112] J. Bartel, M. Brack and M. Durand, Regensburg preprint TPR-85-3, submitted to Nucl. Phys. A.

[113] E. Suraud, J. Phys. (Paris) Colloques C6 (1984) 161.

[114] P. Bonche, S. Levit and D. Vautherin, Nucl. Phys. A427 (1984) 278; A436 (1985) 265.

[115] R.W. Hasse and W. Stocker, Phys. Lett. 44B (1973) 26.

[116] C.J. Pethick, D.G. Ravenhall and J.M. Lattimer, Phys. Lett. 128B (1983) 137.

[117] W. Stocker, Phys. Lett. 142B (1984) 319.

[118] B.K. Jennings, Phys. Lett. 74B (1978) 13.

[119] J.W. Negele and D. Vautherin, Phys. Rev. C5 (1972) 1472

[120] M. Rayet, M. Arnould, F. Tondeur and G. Paulus, Astron. Astrophys. 116 (1982) 183.

[121] M. Farine, Z. Physik A320 (1985) 337.

[122] M. Asghar and R.W. Hasse, J. Phys. (Paris) Colloques C6 (1984) 455.

\section{Note added in proof}

While this review was in print, the fourth order gradient corrections to the functionals $\tau_{\mathrm{ETF}}[\rho]$ and $\sigma_{\mathrm{ETF}}[\rho]$ have been derived in ref. [112] for the case of a local potential. As anticipated in section 6.2, these terms have the correct limits for $T \rightarrow 0$, i.e. $\sigma_{4}[\rho] \rightarrow 0$ and $\tau_{4}^{T}[\rho] \rightarrow \tau_{4}[\rho]$, eq. (A.1). Also in ref. [112], the Euler variational equation including all fourth order terms has been solved exactly for semi-infinite symmetric nuclear matter at $T \geq 0$ in the case of phase equilibrium. It was found that the asymmetric Fermi-type trial densities eq. (6.69) (with optimized $\alpha$ and $\gamma$ at each temperature) constitute excellent approximations to the exact variational densities. The LDM parameters $a_{\mathrm{s}}$ and $a_{\mathrm{c}}$ found in the present work (see table 8 and fig. 21) agree with those calculated from the exact densities in ref. [112] for all interactions within less than $1 \%$ for $0 \leq T \leq 2 \mathrm{MeV}$ and within less than $1 \%$ for $T \geq 5 \mathrm{MeV}$. This gives a nice a posterior confirmation of the restricted variational approach used in the present work. 